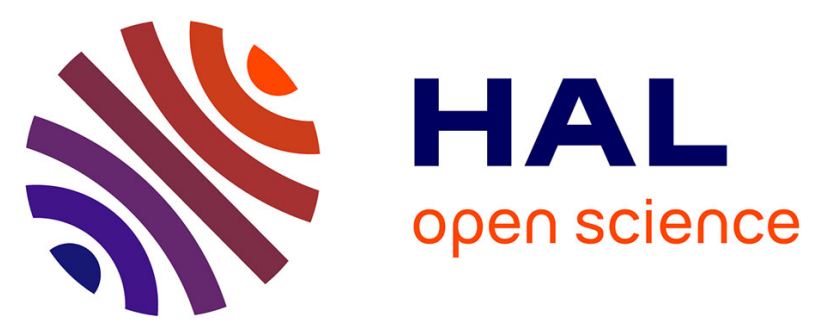

\title{
State observers of a vascular fluid-structure interaction model through measurements in the solid
}

\author{
Cristobal Bertoglio, Dominique Chapelle, Miguel Angel Fernández, \\ Jean-Frédéric Gerbeau, Philippe Moireau
}

\section{- To cite this version:}

Cristobal Bertoglio, Dominique Chapelle, Miguel Angel Fernández, Jean-Frédéric Gerbeau, Philippe Moireau. State observers of a vascular fluid-structure interaction model through measurements in the solid. Computer Methods in Applied Mechanics and Engineering, 2013, 256, pp.149-168. 10.1016/j.cma.2012.12.010 . hal-00764332v3

\section{HAL Id: hal-00764332 \\ https://hal.inria.fr/hal-00764332v3}

Submitted on 28 Dec 2012

HAL is a multi-disciplinary open access archive for the deposit and dissemination of scientific research documents, whether they are published or not. The documents may come from teaching and research institutions in France or abroad, or from public or private research centers.
L'archive ouverte pluridisciplinaire HAL, est destinée au dépôt et à la diffusion de documents scientifiques de niveau recherche, publiés ou non, émanant des établissements d'enseignement et de recherche français ou étrangers, des laboratoires publics ou privés. 


\section{State observers of a} vascular fluid-structure interaction model through measurements in the solid

C. Bertoglio, D. Chapelle, M.A. Fernández, J.-F. Gerbeau, P. Moireau

\section{RESEARCH} REPORT

$\mathbf{N}^{\circ} 8177$

December 2012 



\title{
State observers of a vascular fluid-structure interaction model through measurements in the solid
}

\author{
C. Bertoglio *, D. Chapelle`, M.A. Fernández*, J.-F. Gerbeau*, \\ P. Moireau ${ }^{\dagger}$
}

Project-Teams Macs and Reo

Research Report n ${ }^{\circ} 8177$ - December 2012 - 47 pages

\begin{abstract}
We analyze the performances of two types of Luenberger observers - namely, the so-called Direct Velocity Feedback and Schur Displacement Feedback procedures, originally devised for elasto-dynamics - to estimate the state of a fluid-structure interaction model for hemodynamics, when the measurements are assumed to be restricted to displacements or velocities in the solid. We first assess the observers using hemodynamics-inspired test problems with the complete model, including the Navier-Stokes equations in Arbitrary Lagrangian-Eulerian formulation, in particular. Then, in order to obtain more detailed insight we consider several well-chosen simplified models, each of which allowing a thorough analysis - emphasizing spectral considerations - while illustrating a major phenomenon of interest for the observer performance, namely, the added mass effect for the structure, the coupling with a lumped-parameter boundary condition model for the fluid flow, and the fluid dynamics effect per se. Whereas improvements can be sought when additional measurements are available in the fluid domain in order to more effectively deal with strong uncertainties in the fluid state, in the present framework this establishes Luenberger observer methods as very attractive strategies - compared, e.g. to classical variational techniques - to perform state estimation, and more generally for uncertainty estimation since other observer procedures can be conveniently combined to estimate uncertain parameters.
\end{abstract}

Key-words: estimation, observers, fluid-structure interaction, hemodynamics.

* Project-Team Reo, Inria Paris-Rocquencourt, France

$\dagger$ Project-Team Macs, Inria Saclay-Île de France, France

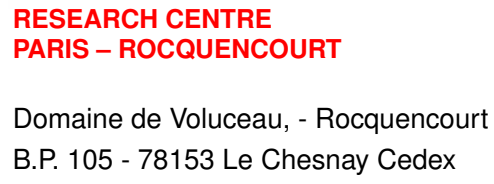




\section{Observateurs d'état d'un modèle d'interaction fluide-structure vasculaire par des mesures dans le solide}

Résumé : Nous analysons les performances de deux observateurs de type Luenberger (Direct Velocity Feedback et Schur Displacement Feedback, conçus à lorigine pour l'élasto-dynamique) pour estimer l'état d'un modèle d'interaction fluide-structure en hémodynamique, lorsque les mesures sont supposées être limitées à des déplacements ou des vitesses dans le solide. Nous évaluons d'abord les observateurs à l'aide de cas-tests (inspirés de l'hémodynamique) avec le modèle complet, comportant les équations de Navier-Stokes en formulation arbitrairement LagrangienneEulérienne, en particulier. Ensuite, afin d'obtenir un aperçu plus détaillé nous considérons plusieurs modèles simplifiés, chacun permetant une analyse plus approfondie (en mettant l'accent sur des considérations spectrales) tout en illustrant un phénomène d'intérêt majeur pour la performance des observateurs: l'effet de masse ajoutée pour le structure, le couplage avec un modèle réduit de condition au limite pour le fluide et l'effet de la dynamique fluide en soi, par exemple. Même si des améliorations additionnelles peuvent être envisagées, pour réduire plus efficacement les fortes incertitudes sur l'état fluide lorsque des mesures supplémentaires sont disponibles dans le fluide, le cadre actuel permet de conclure que les observateurs de type Luenberger sont des méthodes très attractivers (par rapport aux techniques variationnelles classiques, par exemple) pour l'estimation d'état et, plus généralement, pour l'estimation d'incertitudes, car d'autres observateurs peuvent être facilement combinés pour estimer les paramètres incertains.

Mots-clés : estimation, observateurs, interaction fluide-structure, hémodynamique. 


\section{Introduction}

During the last decade, the simulation of blood flows in compliant vessels has raised increasing attention for the potential it offers to clinicians to better comprehend the cardiovascular system and predict pathologies progression and treatment outcome. However, despite important advances on numerical algorithms (see, e.g., [30, 24, 1, 28, 35, 20, 27] and the references therein), the personalization of the simulations to a specific patient and condition is still a challenge that needs to be addressed in order to make modeling applicable in the clinical context. This personalization can be performed in the framework of data assimilation by reducing the uncertainties of a model using partial observations of the system of interest extracted, here, from medical imaging and signals recordings.

Classically, data assimilation of distributed parameter systems (like those arising from the discretization of partial-differential equations) is performed within a variational approach, i.e., by iteratively minimizing a least squares criterion which balances the error between observations and model prediction, with a regularization term based on some a priori knowledge on the solution (see, e.g., [3]). One of the main difficulties of this approach lies in the computation of the gradient of the functional - typically adjoint-based - which involves major implementation efforts. Moreover, it usually requires many (sequential) solutions of the forward problem and the storage of the state of the system at all times, leading to a prohibitive computational cost for large, multiphysics problems as in fluid-structure simulations in hemodynamics. Hence, previous variational data assimilation trials in this context were mostly based on simplified models [44, 61], and for 3D problems in [22] by modifying the minimization problem in order to avoid the resolution of the adjoint equations.

In this work, we consider another family of methods to perform data assimilation, the socalled sequential or filtering approaches, by which the model prediction is improved sequentially - i.e. all along the simulation - by confronting the available observations to the current simulation output. The correction is inferred by applying a linear operator, named the filter, to the discrepancy between the model prediction and the available measurements. In particular, the Kalman filter satisfies an optimality criterion [7], but this leads to operations with full matrices of the size of the system state, which makes Kalman filtering per se not tractable for distributed parameter systems. However, some effective sequential procedures for mechanical systems have been recently introduced: in [51, 52] for state estimation inspired from Luenberger observer concepts [46], and for parameter identification in [51, 50]. In particular, in [50], the authors formulate a general reduced-order version of the Unscented Kalman Filter (UKF) [41], extending the Singular Evolutive Extended Kalman filter (SEIK) proposed in [57], and proceed to apply this method to the estimation of solid mechanics constitutive parameters. Unlike variational approaches, see for instance [56, 2], this algorithm does not need adjoint or tangent operators and can be very easily parallelized. As a result, the data assimilation computation time is comparable to that of a standard simulation of the model. Moreover, the implementation requires only superficial modifications in existing solvers.

This reduced-order UKF strategy was already applied in [8, 9] for the estimation of uncertain parameters in three-dimensional fluid-structure systems. Specifically, this addressed the determination of heterogeneous values of Young's modulus in the structure, and the estimation of the fluid proximal resistance, both from measurements of the displacements at the fluid-structure interface. Moreover, in [49] this technique was applied to the estimation of solid boundary support parameters of a whole aortic FSI-model with real patient data. To the authors knowledge, this is the first reported data-assimilation trial in fully three-dimensional fluid-structure problems for hemodynamics with real data, albeit without a detailed theoretical analysis.

In all these studies except for [49], a perfectly known initial condition is assumed in order to 
simplify the estimation problem. Nevertheless, it was shown in [9] that even a small error in the initial condition can dramatically affect the parameter identification, see also [51] for a detailed discussion. In practice, this initial condition uncertainty arises for example when only a part of initial state is observed (i.e. when some quantities are not directly measured). Also, because any parameter or modeling errors deviate the model trajectory from the real state, this induces state errors similar to initial condition errors at later times. Therefore, the purpose of state estimation is to stabilize the model close to the actual trajectory by applying measurement-based corrections.

In this context, we investigate here the extension to FSI state estimation of the Luenberger observers proposed in $[51,52]$ for solid mechanics. The main objective is to construct a tractable and effective state estimator - or observer - for FSI using data processed from rather standard medical imaging. In order to focus on the specificity of the design for the physics and observations types of concern, we assume an uncertainty in the initial condition only. In addition, we consider linear observation operators - namely, Lagrangian displacements on a solid subdomain or at the fluid-structure interface. Nevertheless, the ultimate goal is to combine these state estimators with the reduced-order UKF used in [9] for improving the joint state and parameter estimation in FSI problems.

The rest of the paper is organized as follows. In Section 2 we present the fluid-structure observers based on displacement and velocity measurements of the vessel wall, as well as some numerical experiments to assess their efficiency in circumventing an error in the initial condition. Then, in Section 3 we perform a thorough analysis of the observers using simplified problems in order to explain the different behaviors observed in Section 2, and we propose a new filter especially designed for FSI problems with a strong added-mass effect. We end with some concluding remarks in Section 5.

\section{Observer for the fluid-structure interaction problem}

In this section we introduce the formulations for the fluid-structure observers when velocities or displacements are available in a subpart of the solid domain. We start with a reminder of the fluid-structure equations. Then, we discuss different options on how to define the discrepancy between observations and model for the two types of measurements. Next, we present the respective observers, and we conclude with some numerical experiments in order to illustrate their performance.

\subsection{Fluid-structure interaction equations}

We consider the mechanical interaction between an incompressible fluid and an elastic structure. The fluid is described by the Navier-Stokes equations, in a moving domain $\Omega^{\mathrm{f}}(t) \subset \mathbb{R}^{d}$, $d=2,3$ and the structure by the linear elasticity equations in $\Omega_{0}^{\mathrm{s}} \subset \mathbb{R}^{d}$, with $\Omega_{0}^{\mathrm{s}}=\Omega^{\mathrm{s}}(0)$ and $\Omega^{\mathrm{s}}(t)$ the deformed solid domain at time $t$. The motion of the fluid domain is parametrized in terms of the Arbitrary Lagrangian Eulerian (ALE) map $\phi_{\text {ale }}: \Omega_{0}^{\mathrm{f}} \times \mathbb{R}^{+} \rightarrow \mathbb{R}^{d}$ (see Figure 1 ), hence $\Omega^{\mathrm{f}}(t)=\phi_{\text {ale }}\left(\Omega_{0}^{\mathrm{f}}, t\right)$. The displacement of the fluid domain $\boldsymbol{y}_{\mathrm{f}}: \Omega_{0}^{\mathrm{f}} \times \mathbb{R}^{+} \rightarrow \mathbb{R}^{d}$ is defined by the relation $\boldsymbol{y}(\boldsymbol{\xi}, t)=\phi_{\text {ale }}(\boldsymbol{\xi}, t)-\boldsymbol{\xi}, \forall \boldsymbol{\xi} \in \Omega_{0}^{\mathrm{f}}$. The current and reference configurations of the fluid-structure interface are denoted by $\Sigma=\partial \Omega^{\mathrm{s}}(t) \cap \partial \Omega^{\mathrm{f}}(t)$ and $\Sigma_{0}=\partial \Omega_{0}^{\mathrm{s}} \cap \partial \Omega_{0}^{\mathrm{f}}$, respectively. Moreover, we consider the following partitions of the fluid and solid boundaries, $\partial \Omega^{\mathrm{f}}(t)=\Gamma^{\text {in }} \cup \Gamma^{\text {out }} \cup \Sigma(t)$ and $\partial \Omega_{0}^{\mathrm{s}}=\Gamma_{0}^{\mathrm{d}} \cup \Gamma_{0}^{\mathrm{n}} \cup \Sigma_{0}$ (see Figure 1 ).

The coupled FSI problem reads as follows. For $t>0$, find the fluid velocity $\boldsymbol{u}_{\mathrm{f}}: \Omega_{0}^{\mathrm{f}} \times \mathbb{R}^{+} \rightarrow \mathbb{R}^{d}$, the fluid pressure $p: \Omega_{0}^{\mathrm{f}} \times \mathbb{R}^{+} \rightarrow \mathbb{R}$, the fluid domain displacement $\boldsymbol{y}_{\mathrm{f}}: \Omega_{0}^{\mathrm{f}} \times \mathbb{R}^{+} \rightarrow \mathbb{R}^{d}$, the structure displacement $\boldsymbol{y}_{\mathrm{s}}: \Omega_{0}^{\mathrm{s}} \times \mathbb{R}^{+} \rightarrow \mathbb{R}^{d}$ and structure velocity $\boldsymbol{u}_{\mathrm{s}}: \Omega_{0}^{\mathrm{s}} \times \mathbb{R}^{+} \rightarrow \mathbb{R}^{d}$ such that 
- Fluid equations (ALE formalism):

$$
\left\{\begin{aligned}
\left.\rho_{\mathrm{f}} \frac{\partial \boldsymbol{u}_{\mathrm{f}}}{\partial t}\right|_{\boldsymbol{\xi}}+\rho_{\mathrm{f}}\left(\boldsymbol{u}_{\mathrm{f}}-\boldsymbol{w}\right) \cdot \boldsymbol{\nabla} \boldsymbol{u}_{\mathrm{f}}-\boldsymbol{\nabla} \cdot \boldsymbol{\sigma}_{\mathrm{f}}\left(\boldsymbol{u}_{\mathrm{f}}, p\right)=\mathbf{0}, & \text { in } \Omega^{\mathrm{f}}(t) \\
\boldsymbol{\nabla} \cdot \boldsymbol{u}_{\mathrm{f}}=0, & \text { in } \Omega^{\mathrm{f}}(t) \\
\boldsymbol{u}_{\mathrm{f}}=\boldsymbol{u}_{\text {in }}, & \text { on } \Gamma^{\text {in }} \\
\boldsymbol{\sigma}_{\mathrm{f}}\left(\boldsymbol{u}_{\mathrm{f}}, p\right) \cdot \boldsymbol{n}_{\mathrm{f}}=-\bar{p} \boldsymbol{n}_{\mathrm{f}}, & \text { on } \Gamma^{\text {out }}
\end{aligned}\right.
$$

with $\boldsymbol{\sigma}_{\mathrm{f}}\left(\boldsymbol{u}_{\mathrm{f}}, p\right)=-p \mathbb{1}+2 \mu_{\mathrm{f}} \boldsymbol{\varepsilon}\left(\boldsymbol{u}_{\mathrm{f}}\right)$, where $\boldsymbol{\varepsilon}\left(\boldsymbol{u}_{\mathrm{f}}\right)$ denotes the deformation rate tensor, $\mu_{\mathrm{f}}$ the dynamic viscosity, and $\left.\frac{\partial}{\partial t}\right|_{\xi}$ the ALE derivative (see, e.g., [28]). In the hemodynamics problems considered in this work, the outlet pressure $\bar{p}$ is obtained by solving the differential-algebraic equation

$$
\left\{\begin{array}{l}
\bar{p}=\pi+R_{\mathrm{p}} Q \\
C \frac{d \pi}{d t}+\frac{\pi}{R_{\mathrm{d}}}=Q \\
Q=\int_{\Gamma^{\text {out }}} \boldsymbol{u}_{\mathrm{f}} \cdot \boldsymbol{n}_{\mathrm{f}} d \Gamma
\end{array}\right.
$$

where $\pi$ represents a distal pressure. Here, the distal resistance $R_{\mathrm{d}}$, the proximal resistance $R_{\mathrm{p}}$ and the capacitance $C$ are assumed to be given. The "zero-dimensional" equation (1b) is known as the three-element Windkessel model (see for example [55]). It represents the flow resistance $\left(R_{\mathrm{p}}\right.$ and $R_{\mathrm{d}}$ ) and the compliance $(C)$ of the vessels beyond the $3 \mathrm{D}$ portion modeled by (1a).

- Structure equations:

$$
\left\{\begin{aligned}
\partial_{t} \boldsymbol{y}_{\mathrm{s}}=\boldsymbol{u}_{\mathrm{s}}, & \text { in } \Omega_{0}^{\mathrm{s}} \\
\rho_{\mathrm{s}} \partial_{t} \boldsymbol{u}_{\mathrm{s}}-\eta_{\mathrm{s}} \boldsymbol{\nabla} \cdot \boldsymbol{\sigma}_{\mathrm{s}}\left(\boldsymbol{u}_{\mathrm{s}}\right)-\boldsymbol{\nabla} \cdot \boldsymbol{\sigma}_{\mathrm{s}}\left(\boldsymbol{y}_{\mathrm{s}}\right)=\mathbf{0}, & \text { in } \Omega_{0}^{\mathrm{s}} \\
\boldsymbol{y}_{\mathrm{s}}=\mathbf{0}, & \text { on } \Gamma_{0}^{\mathrm{d}} \\
\eta_{\mathrm{s}} \boldsymbol{\sigma}_{\mathrm{s}}\left(\boldsymbol{u}_{\mathrm{s}}\right) \cdot \boldsymbol{n}_{\mathrm{s}}+\boldsymbol{\sigma}_{\mathrm{s}}\left(\boldsymbol{y}_{\mathrm{s}}\right) \cdot \boldsymbol{n}_{\mathrm{s}}=\mathbf{0}, & \text { on } \Gamma_{0}^{\mathrm{n}}
\end{aligned}\right.
$$

with $\boldsymbol{\sigma}_{s}\left(\boldsymbol{y}_{\mathrm{s}}\right)=\lambda_{\mathrm{s}} \operatorname{Tr}\left(\boldsymbol{\varepsilon}\left(\boldsymbol{y}_{\mathrm{s}}\right)\right) \mathbb{1}+2 \mu_{\mathrm{s}} \varepsilon\left(\boldsymbol{y}_{\mathrm{s}}\right)$ where, $\lambda_{s}$ and $\mu_{\mathrm{s}}$ are the Lamé constants and $\eta_{s}$ is a viscous modulus.

- Coupling conditions:

$$
\left\{\begin{array}{rr}
\boldsymbol{y}_{\mathrm{f}}=\operatorname{Ext}_{\Sigma_{0}}^{\mathrm{f}}\left(\left.\boldsymbol{y}_{\mathrm{s}}\right|_{\Sigma_{0}}\right), \quad \boldsymbol{w}=\partial_{t} \boldsymbol{y}_{\mathrm{f}}, \quad \Omega^{\mathrm{f}}(t)=\left(I_{\Omega^{\mathrm{f}}}+\boldsymbol{y}_{\mathrm{f}}\right)\left(\Omega_{0}^{\mathrm{f}}, t\right) \\
\boldsymbol{u}_{\mathrm{f}}=\boldsymbol{u}_{\mathrm{s}}, \quad \text { on } \Sigma(t) \\
\eta_{\mathrm{s}} \boldsymbol{\sigma}_{\mathrm{s}}\left(\boldsymbol{u}_{\mathrm{s}}\right) \cdot \boldsymbol{n}_{\mathrm{s}}+\boldsymbol{\sigma}_{\mathrm{s}}\left(\boldsymbol{y}_{\mathrm{s}}\right) \cdot \boldsymbol{n}_{\mathrm{s}}+J_{\mathrm{f}} \boldsymbol{\sigma}_{\mathrm{f}}\left(\boldsymbol{u}_{\mathrm{f}}, p\right) \cdot \boldsymbol{F}_{\mathrm{f}}^{-T} \cdot \boldsymbol{n}_{\mathrm{f}}=\mathbf{0}, \quad \text { on } \Sigma_{0}
\end{array}\right.
$$

with $\operatorname{Ext}_{\Sigma_{0}}^{\mathrm{f}}$ an extension operator from $\Sigma_{0}$ to $\Omega_{0}^{\mathrm{f}}, \boldsymbol{F}_{\mathrm{f}}$ the deformation gradient and $J_{\mathrm{f}}=$ $\operatorname{det} \boldsymbol{F}_{\mathrm{f}}$.

This problem is completed with appropriate initial conditions: velocity $\boldsymbol{u}_{\mathrm{f}}(0)$, domain displacement $\boldsymbol{y}_{\mathrm{f}}(0)$ and Windkessel pressure $\pi(0)$ for the fluid, initial velocity $\boldsymbol{u}_{\mathrm{s}}(0)$ and displacement $\boldsymbol{y}_{\mathrm{s}}(0)$ for the solid.

$\mathrm{RR} \mathrm{n}^{\circ} 8177$ 


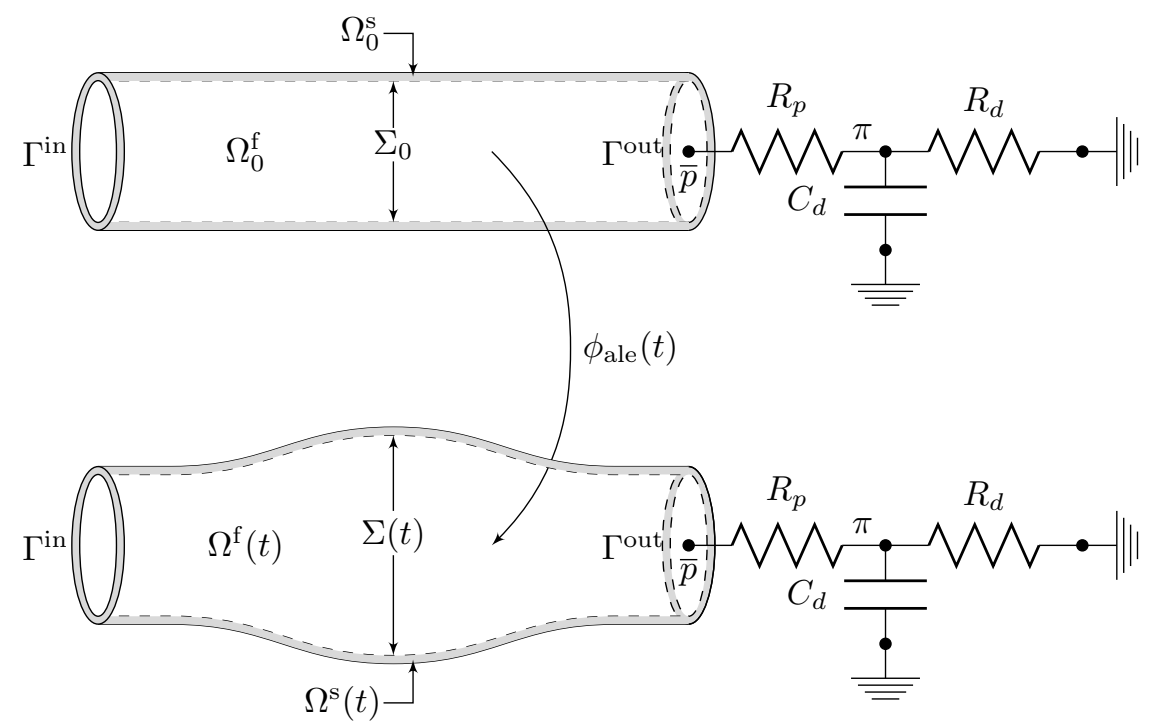

Figure 1: Reference (top) and current domain (bottom) in the ALE formulation

Energy balance. In what follows, the quantity

$$
E(t)=\underbrace{\frac{\rho_{\mathrm{f}}}{2}\left\|\boldsymbol{u}_{\mathrm{f}}\right\|_{L^{2}\left(\Omega_{\mathrm{f}}(t)\right)}^{2}+\frac{\rho_{\mathrm{s}}}{2}\left\|\boldsymbol{u}_{\mathrm{s}}\right\|_{L^{2}\left(\Omega_{0}^{\mathrm{s}}\right)}^{2}}_{\text {Kinetic energy }}+\underbrace{\frac{1}{2}\left\|\boldsymbol{y}_{\mathrm{s}}\right\|_{\mathcal{E}_{l}^{s}}^{2}+\frac{C}{2} \pi^{2}}_{\text {Potential energy }}
$$

denotes the total mechanical energy of the fluid-structure system described by (1), with $\|\cdot\|_{\mathcal{E}_{l}^{s}}$ standing for the elastic energy-norm of the structure, i.e.,

$$
\left\|\boldsymbol{d}_{\mathrm{s}}\right\|_{\mathcal{E}_{l}^{s}}=\sqrt{\left\langle\boldsymbol{d}_{\mathrm{s}}, \boldsymbol{d}_{\mathrm{s}}\right\rangle_{\mathcal{E}_{l}^{s}}}, \quad\left\langle\boldsymbol{d}_{\mathrm{s}}, \boldsymbol{v}_{\mathrm{s}}\right\rangle_{\mathcal{E}_{l}^{s}}=\int_{\Omega_{0}^{\mathrm{s}}} \boldsymbol{\sigma}_{\mathrm{s}}\left(\boldsymbol{d}_{\mathrm{s}}\right): \boldsymbol{\varepsilon}_{\mathrm{s}}\left(\boldsymbol{v}_{\mathrm{s}}\right) d \Omega
$$

for all $\boldsymbol{d}_{\mathrm{s}}, \boldsymbol{v}_{\mathrm{s}} \in \mathcal{V}_{s}^{d}$, a suitable space of displacement test functions.

The next result extends the classical energy estimate for the coupled system (1a), (1c), (1d) (see, e.g., [28]) by incorporating the Windkessel component (1b). As expected, dissipation only comes from the viscosity in the fluid and the solid, and from the resistive terms in the outlet boundary conditions. In particular, the powers exchanged by the fluid and the structure are exactly balanced at the interface, as a direct consequence of the coupling conditions (1d).

\section{Proposition 1}

The following identity holds for $t>0$ :

$$
\frac{\mathrm{d}}{\mathrm{d} t} E(t)=-D(t)-\int_{\Gamma^{\text {in }}} \frac{\rho_{\mathrm{f}}}{2} \boldsymbol{u}_{\text {in }} \cdot \boldsymbol{n}_{\mathrm{f}}\left|\boldsymbol{u}_{\text {in }}\right|^{2}-\int_{\Gamma_{\text {out }}} \frac{\rho_{\mathrm{f}}}{2} \boldsymbol{u}_{\mathrm{f}} \cdot \boldsymbol{n}_{\mathrm{f}}\left|\boldsymbol{u}_{\mathrm{f}}\right|^{2} d \Gamma,
$$

with

$$
D(t)=2 \mu_{\mathrm{f}}\left\|\varepsilon\left(\boldsymbol{u}_{\mathrm{f}}\right)\right\|_{L^{2}\left(\Omega_{\mathrm{f}}(t)\right)}^{2}+\eta_{\mathrm{s}}\left\|\boldsymbol{u}_{\mathrm{s}}\right\|_{\mathcal{E}_{l}^{s}}^{2}+\frac{\pi^{2}}{R_{\mathrm{d}}}+R_{\mathrm{p}} Q^{2} .
$$


Proof. The identity (2) can be derived from a standard energy argument (see, e.g., [28]). We first multiply $(1 \mathrm{a})_{1}$ by $\boldsymbol{u}_{\mathrm{f}}$, integrate over $\Omega_{\mathrm{f}}(t)$ and apply the Green formula to the divergence term. Similarly, we multiply $(1 \mathrm{c})_{2}$ by $\boldsymbol{u}_{\mathrm{s}}$, integrate over $\Omega_{0}^{\mathrm{s}}$ and apply the Green formula once more. By adding the resulting expressions and using (1d) and (1b) we get (2).

\section{REMARK 1}

It is well-known that the flux of kinetic energy at the outlet, i.e., the last term in (2), can have a destabilizing effect in presence of backflows. None of the simulations presented in this paper is affected by these instabilities. This issue will therefore not be addressed here, but we may refer to $[5,43,36]$ for stabilization techniques (and to [26] for a comparison of some of these methods in hemodynamics), and to [33] for different 3D-1D coupling conditions.

State-space form. Finally we introduce the standard state-space representation of System (1) classically used to present control and observation problems [19,21]. First we introduce the state of the system as the combination $x=\left(\boldsymbol{y}_{\mathrm{s}}, \boldsymbol{u}_{\mathrm{s}}, \boldsymbol{y}_{\mathrm{f}}, \boldsymbol{u}_{\mathrm{f}}, \pi\right)$. Then, we can formally define an evolution operator $\mathcal{A}$ - nonlinear in our case - such that System (1) reads

$$
\left\{\begin{array}{l}
\dot{x}=\mathcal{A}(x) \\
x(0)=x_{0}+\zeta
\end{array}\right.
$$

where $\zeta$ refers to an unknown component of the initial condition. Note that the distributed fluid pressure does not appear directly in (3) since it corresponds to a Lagrange multiplier of the fluid incompressibility constraint prescribed through the fluid space definition.

\subsection{Observer based on solid measurements}

We now present the fluid-structure observer based on measurements in the solid. We begin by defining the types of discrepancy operators and detailing the filtering setting. Then we present the specific formulations for the velocities- and displacements-based observers.

\subsubsection{Discrepancy measure minimization}

A physical system like blood flow in a compliant artery can be observed through various measurement modalities: artery wall motion obtained from $3 \mathrm{D}+$ time medical imaging - computed tomography (CT), magnetic resonance imaging (MRI) or ultrasound (US) - cross section blood flow rates by Phase Contrast MRI or Doppler US, local catheter-measured pressures, and so on. These measurements are usually restricted to a limited number of physical quantities and locations in space. However, they are available during the complete simulation time even if, in the case of cardiovascular data acquisition, they are aggregated over different cardiac cycles based, e.g., on electrocardiogram gating. They are of course subject to noise, and their post-processing can introduce some further inaccuracies.

Usually, computational models only use the data to define the initial and the boundary conditions. By contrast, an observer is a system which can benefit from the whole set of data to improve the result of the model. For example, the data are used in [51] to reduce the error in the initial condition for an elasticity problem, and in [17] to reduce the discretization error for a wave-like equation.

In the cardiovascular context, the most common type of non-invasive measurements is provided by medical images which contain information about the solid kinematics. In [52], this type of measurements is used to define an observer for a solid problem. Here, we aim at extending this 
methodology to an FSI system. In this study, we limit ourselves to measurements of structure displacements or velocities. But the observers presented here can be extended to more realistic measurements, like for example the distance to segmented surfaces. This has been proposed in [52] for solid mechanics problems, applied in [15] with MRI images, and in [49] with CT images for an FSI problem - but without detailed mathematical analysis in this latter context.

In the present work, we will consider measurements defined for example by

$$
\boldsymbol{z}_{d}=\mathcal{H}_{d} \boldsymbol{y}_{\mathrm{s}}^{\mathrm{ref}}
$$

where $\mathcal{H}_{d}$ stands for the observation operator which, e.g., selects the field $\boldsymbol{y}_{\mathrm{s}}$ in a subdomain $\Omega_{\mathrm{m}} \in \Omega_{\mathrm{s}}$ or the trace of the field on the fluid-structure interface $\Sigma_{0}$. In these cases, we have

$$
\boldsymbol{z}_{d}=\boldsymbol{y}_{\mathrm{s} \mid \Omega_{\mathrm{m}}}^{\mathrm{ref}}
$$

or

$$
\boldsymbol{z}_{d}=\boldsymbol{y}_{\mathrm{s} \mid \Sigma_{0}}^{\mathrm{ref}} .
$$

Even if velocity measurements are rarely directly accessible, we will also consider

$$
\boldsymbol{z}_{v}=\mathcal{H}_{v} \boldsymbol{u}_{\mathrm{s}}^{\mathrm{ref}}
$$

because this kind of observation can be helpful to understand how to derive adequate observers.

When these two types of measurements are simultaneously available, the operators can be aggregated to obtain

$$
z=\left(\begin{array}{c}
\boldsymbol{z}_{d} \\
\boldsymbol{z}_{v}
\end{array}\right)=\left(\begin{array}{cc}
\mathcal{H}_{d} & 0 \\
0 & \mathcal{H}_{v}
\end{array}\right)\left(\begin{array}{l}
\boldsymbol{y}_{\mathrm{s}}^{\mathrm{ref}} \\
\boldsymbol{u}_{\mathrm{s}}^{\mathrm{ref}}
\end{array}\right)
$$

More generally for $x^{\text {ref }}=\left(\boldsymbol{y}_{\mathrm{s}}^{\text {ref }}, \boldsymbol{u}_{\mathrm{s}}^{\text {ref }}, \boldsymbol{y}_{\mathrm{f}}^{\text {ref }}, \boldsymbol{u}_{\mathrm{f}}^{\text {ref }}, \pi^{\text {ref }}\right)$, we can define

$$
z=\mathcal{H} x^{\text {ref }}+\chi
$$

with $\chi$ some additive measurement noise. Note that, in this formalism, measurements of the fluid velocity $\boldsymbol{u}_{\mathrm{f}}$ or Windkessel pressure $(\bar{p}$ or $\pi)$ can also be considered but they are not the focus of this paper. By contrast, measurements of the fluid pressure field $p$ do not directly fit into this formalism because the pressure is not a state variable in our problem.

The principle of optimal filtering in data assimilation - with Kalman filtering [42] and various extensions thereof [60] - is to minimize in time - up to additive regularization terms - a discrepancy measure comparing a given state $x=\left(\boldsymbol{y}_{\mathrm{s}}, \boldsymbol{u}_{\mathrm{s}}, \boldsymbol{y}_{\mathrm{f}}, \boldsymbol{u}_{\mathrm{f}}, \pi\right)$ with the measurements $z$. Considering again the example (5), the discrepancy measure can be

$$
\operatorname{meas}_{L^{2}\left(\Omega_{\mathrm{m}}\right)}\{x, z\}(t)=\frac{1}{2} \int_{\Omega_{\mathrm{m}}}\left|\boldsymbol{z}_{d}-\boldsymbol{y}_{\mathrm{s}}\right|^{2} d \Omega,
$$

or

$$
\operatorname{meas}_{L^{2}\left(\Sigma_{0}\right)}\{x, z\}(t)=\frac{1}{2} \int_{\Sigma_{0}}\left|\boldsymbol{z}_{d}-\boldsymbol{y}_{\mathrm{s}}\right|^{2} d \Gamma,
$$

when choosing an $L^{2}$-norm for comparing the two fields. Alternatively, other norms can be chosen, for instance on the boundary we can consider an $H^{\frac{1}{2}}\left(\Sigma_{0}\right)$ type norm, while for a subdomain we can employ an $H^{1}\left(\Omega_{\mathrm{m}}\right)$-norm. Then, we use

$$
\operatorname{meas}_{H^{1}\left(\Omega_{\mathrm{m}}\right)}\{x, z\}(t)=\frac{1}{2}\left\|\operatorname{Ext}_{\Omega_{\mathrm{m}}}^{\mathrm{s}}\left(\boldsymbol{z}-\boldsymbol{y}_{\mathrm{s} \mid \Omega_{\mathrm{m}}}\right)\right\|_{H_{1}\left(\Omega_{0}^{\mathrm{s}}\right)}^{2},
$$

or

$$
\operatorname{meas}_{H^{1 / 2}\left(\Sigma_{0}\right)}\{x, z\}(t)=\frac{1}{2}\left\|\operatorname{Ext}_{\Sigma_{0}}^{\mathrm{s}}\left(\boldsymbol{z}-\boldsymbol{y}_{\mathrm{s} \mid \Sigma_{0}}\right)\right\|_{H_{1}\left(\Omega_{0}^{\mathrm{s}}\right)}^{2},
$$


where $\operatorname{Ext}_{\Sigma_{0}}^{s}$ and $\operatorname{Ext}_{\Omega_{\mathrm{m}}}^{s}$ are extension operators defined in the structure domain and based on the elasticity formulation. The advantage of the state space form is to summarize all these discrepancy measures in

$$
\operatorname{meas}_{\mathcal{M}}\{x, z\}(t)=\frac{1}{2}\|z-\mathcal{H} x\|_{\mathcal{M}}^{2},
$$

for a given norm $\mathcal{M}$. Therefore, optimal filters are built by considering the minimization of a least square criterion

$$
J(x(0), T)=\frac{1}{2}\left\|x(0)-x_{0}\right\|_{\mathcal{E}}^{2}+\frac{1}{2} \int_{0}^{T}\|z(s)-\mathcal{H} x(s)\|_{\mathcal{M}}^{2} d s,
$$

with the dynamics of $x(\cdot)$ given by (3). The norm $\|\cdot\|_{\mathcal{E}}$ is typically an energy norm naturally associated with the system, and we minimize on the initial condition which entirely determines the rest of the trajectory.

Then the optimal observer - denoted by $\hat{x}$ since it follows its own evolution equation - is defined by

$$
\left\{\begin{array}{l}
\dot{\hat{x}}=\mathcal{A}(\hat{x})+\mathcal{P} \mathcal{H}^{\prime}(z-\mathcal{H} \hat{x}) \\
\hat{x}(0)=x_{0}
\end{array}\right.
$$

where $\mathcal{H}^{\prime}$ is the adjoint of the operator $\mathcal{H}$ with respect to the norm $\mathcal{M}$, and $\mathcal{P}$ an operator deriving from a Ricatti equation in Kalman-like filtering or from a Hamilton-Jacobi-Bellman equation for nonlinear systems [32]. In all cases, these filters suffer from the "curse of dimensionality" as explained by Bellman [6], which makes them intractable with partial differential equations. In [46], Luenberger introduced a new class of observers for which he relaxed the optimality condition to only base the filter design on the requirement that the error system $\tilde{x}=x^{\text {ref }}-\hat{x}$ be asymptotically stable. To that purpose, a possible approach is to differentiate the discrepancy measure (9), namely

$$
\nabla_{x}(\operatorname{meas} \mathcal{M}\{x, z\}(t))=-\mathcal{H}^{\prime}(z-\mathcal{H} x),
$$

and use this gradient as the correction descent direction. Hence, a good observer candidate in state-space form is

$$
\dot{\hat{x}}=\mathcal{A}(\hat{x})+\gamma \mathcal{H}^{\prime}(z-\mathcal{H} \hat{x}),
$$

with $\gamma$ a scalar characterizing the correction intensity. In the case of a linear dynamics operator, $\mathcal{A}(\cdot)=\mathcal{A}_{l} \cdot$, we can derive the dynamics of the error $\tilde{x}=x^{\text {ref }}-\hat{x}$ between the real system $x^{\text {ref }}$ and the observer $\hat{x}$ to get the autonomous system

$$
\dot{\tilde{x}}=\left(\mathcal{A}_{l}-\gamma \mathcal{H}^{\prime} \mathcal{H}\right) \tilde{x}-\gamma \mathcal{H}^{\prime} \chi
$$

Hence, the principle of the observer is to ensure that the semi-group generated by $\mathcal{A}_{l}-\gamma \mathcal{H}^{\prime} \mathcal{H}$ is asymptotically stable, whereas the original dynamics associated with $\mathcal{A}_{l}$ may be intrinsically less stable, or even unstable, as in some fluid-structure systems of interest in the present context, see e.g. [38]. We will analyze our strategy in this light in Section 3, by considering linear fluid models in our coupled fluid-structure problem.

Let us now specify in the next two paragraphs how the strong formulation for the standard FSI problem is modified when constructing an FSI observer in the case of velocities or displacement measurements. 


\subsubsection{Observer based on solid velocities measurements}

We first consider the simplest observer based on solid velocities. Assuming that we measure the velocities in a subdomain $\Omega_{\mathrm{m}}$ or the boundary part $\Sigma_{0}$, we have the following discrepancy measure

$$
\operatorname{meas}_{L^{2}\left(\Omega_{\mathrm{m}}\right)}\{x, z\}(t)=\frac{1}{2} \int_{\Omega_{\mathrm{m}}}\left|\boldsymbol{z}_{v}-\boldsymbol{u}_{\mathrm{s}}\right|^{2} d \Omega,
$$

or

$$
\operatorname{meas}_{L^{2}\left(\Sigma_{0}\right)}\{x, z\}(t)=\frac{1}{2} \int_{\Sigma_{0}}\left|\boldsymbol{z}_{v}-\boldsymbol{u}_{\mathrm{S}}\right|^{2} d \Gamma,
$$

and corresponding tangent operators

$$
\forall \boldsymbol{v}_{\mathrm{s}} \in \mathcal{V}_{s}^{v}, \quad \mathcal{P}_{L^{2}\left(\Omega_{\mathrm{m}}\right)}\{x, z\}(t)\left(\boldsymbol{v}_{\mathrm{s}}\right)=\int_{\Omega_{\mathrm{m}}}\left(\boldsymbol{z}_{v}-\boldsymbol{u}_{\mathrm{s}}\right) \cdot \boldsymbol{v}_{\mathrm{s}} d \Omega,
$$

or

$$
\forall \boldsymbol{v}_{\mathrm{s}} \in \mathcal{V}_{s}^{v}, \quad \mathcal{P}_{L^{2}\left(\Sigma_{0}\right)}\{x, z\}(t)\left(\boldsymbol{v}_{\mathrm{s}}\right)=\int_{\Sigma_{0}}\left(\boldsymbol{z}_{v}-\boldsymbol{u}_{\mathrm{s}}\right) \cdot \boldsymbol{v}_{\mathrm{s}} d \Gamma
$$

In this case, the control operator $\gamma \mathcal{H}^{\prime}$ generates a force proportional to the discrepancy between the computed and measured velocities, so that the tangent expression (13a) or (13b) appears as an external virtual power in the principle of virtual power. The resulting filter is called Direct Velocity Feedback (DVF), widely used in structural control [58], and already applied in [51] for state estimation of solid mechanics systems. The corresponding observer consists in modifying in System (1) the solid formulation (1c) into

$$
\left\{\begin{array}{rr}
\partial_{t} \hat{\boldsymbol{y}}_{\mathrm{s}}=\hat{\boldsymbol{u}}_{\mathrm{s}}, & \text { in } \Omega_{0}^{\mathrm{s}} \\
\rho_{\mathrm{s}} \partial_{t} \hat{\boldsymbol{u}}_{\mathrm{s}}-\eta_{\mathrm{s}} \boldsymbol{\nabla} \cdot \boldsymbol{\sigma}_{\mathrm{s}}\left(\hat{\boldsymbol{u}}_{\mathrm{s}}\right)-\boldsymbol{\nabla} \cdot \boldsymbol{\sigma}_{\mathrm{s}}\left(\hat{\boldsymbol{y}}_{\mathrm{s}}\right)=\gamma_{v} \mathbb{1}_{\Omega_{\mathrm{m}}}\left(\boldsymbol{z}_{v}-\left.\hat{\boldsymbol{u}}_{\mathrm{s}}\right|_{\Omega_{\mathrm{m}}}\right), & \text { in } \Omega_{0}^{\mathrm{s}} \\
\hat{\boldsymbol{y}}_{\mathrm{s}}=\mathbf{0}, & \text { on } \widehat{\Gamma}^{\mathrm{d}} \\
\eta_{\mathrm{s}} \boldsymbol{\sigma}_{\mathrm{s}}\left(\hat{\boldsymbol{u}}_{\mathrm{s}}\right) \cdot \boldsymbol{n}_{\mathrm{s}}+\boldsymbol{\sigma}_{\mathrm{s}}\left(\hat{\boldsymbol{y}}_{\mathrm{s}}\right) \cdot \boldsymbol{n}_{\mathrm{s}}=\mathbf{0}, & \text { on } \widehat{\Gamma}^{\mathrm{n}}
\end{array}\right.
$$

where $\gamma_{v}$ is the gain for the DVF and the notation

$$
\mathbb{1}_{\Omega_{\mathrm{m}}}(\boldsymbol{f})=\left\{\begin{array}{l}
\boldsymbol{f} \text { in } \Omega_{\mathrm{m}} \\
\mathbf{0} \text { otherwise }
\end{array}\right.
$$

for a given vector field $f$ in $\Omega_{\mathrm{m}}$. In the case of measurements on $\Sigma_{0}$, the observer consists now only in changing the coupling conditions (1d) of System (1) into

$$
\left\{\begin{array}{r}
\hat{\boldsymbol{y}}_{\mathrm{f}}=\operatorname{Ext}\left(\hat{\boldsymbol{y}}_{\left.\mathrm{s}\right|_{\Sigma_{0}}}\right), \quad \widehat{\boldsymbol{w}}=\partial_{t} \hat{\boldsymbol{y}}_{\mathrm{f}}, \quad \Omega^{\mathrm{f}}(t)=\left(I_{\Omega_{\mathrm{f}}}+\hat{\boldsymbol{y}}_{\mathrm{f}}\right)\left(\Omega_{0}^{\mathrm{f}}, t\right) \\
\hat{\boldsymbol{u}}_{\mathrm{f}}=\hat{\boldsymbol{u}}_{\mathrm{s}}, \quad \text { on } \Sigma(t) \\
\eta_{\mathrm{s}} \boldsymbol{\sigma}_{\mathrm{s}}\left(\hat{\boldsymbol{u}}_{\mathrm{s}}\right) \cdot \boldsymbol{n}_{\mathrm{s}}+\boldsymbol{\sigma}_{\mathrm{s}}\left(\hat{\boldsymbol{y}}_{\mathrm{s}}\right) \cdot \boldsymbol{n}_{\mathrm{s}}+J_{\mathrm{f}} \boldsymbol{\sigma}_{\mathrm{f}}\left(\hat{\boldsymbol{u}}_{\mathrm{f}}, \hat{p}\right) \cdot \hat{\boldsymbol{F}}_{\mathrm{f}}^{-T} \cdot \boldsymbol{n}_{\mathrm{f}}=\gamma_{v}\left(\boldsymbol{z}_{v}-\hat{\boldsymbol{u}}_{\mathrm{s}}\right), \quad \text { on } \Sigma_{0}
\end{array}\right.
$$

\section{REMARK 2}

The case of velocities measured on a boundary can be intricate when the velocity is only defined in $L^{2}\left(\Omega_{0}^{\mathrm{s}}\right)$ - in particular when no structural damping is present in an elastodynamics system - and therefore does not a priori necessarily have a trace on the boundary. However, assuming this regularity is present in the reference solution - justifying the fact that we can measure it - some hidden regularity can be justified for the observer as recalled in [18] and more precisely discussed in [45]. Moreover, in FSI the trace of the velocity on the interface can be considered because of the improved regularity due to the coupling with the fluid. 
Energetic aspects for the observer - The dissipative character of the DVF filter can be highlighted by a simple energy argument, as in Proposition 1. Thus, for the coupled systems (1a), (1b), (14a), (1d) or (1a), (1b), (1c), (14b) the identity (2) becomes

$$
\frac{\mathrm{d}}{\mathrm{d} t} \hat{E}(t) \leq-\hat{D}(t)-\frac{\gamma_{v}}{2}\left\|\hat{\boldsymbol{u}}_{\mathrm{s}}\right\|_{L^{2}(*)}^{2}+\frac{\gamma_{v}}{2}\left\|\boldsymbol{z}_{v}\right\|_{L^{2}(*)}^{2}-\int_{\Gamma^{\text {in }} \cup \Gamma \text { out }} \frac{\rho_{\mathrm{f}}}{2} \hat{\boldsymbol{u}}_{\mathrm{f}} \cdot \boldsymbol{n}_{\mathrm{f}}\left|\hat{\boldsymbol{u}}_{\mathrm{f}}\right|^{2} d \Gamma,
$$

with the obvious notation

$$
\begin{aligned}
& \hat{E}(t)=\frac{\rho_{\mathrm{f}}}{2}\left\|\hat{\boldsymbol{u}}_{\mathrm{f}}\right\|_{L^{2}\left(\Omega_{\mathrm{f}}(t)\right)}^{2}+\frac{\rho_{\mathrm{s}}}{2}\left\|\hat{\boldsymbol{u}}_{\mathrm{s}}\right\|_{L^{2}\left(\Omega_{0}^{\mathrm{s}}\right)}^{2}+\frac{1}{2}\left\|\hat{\boldsymbol{y}}_{\mathrm{s}}\right\|_{\mathcal{E}_{l}^{s}}^{2}+\frac{C}{2} \hat{\pi}^{2}, \\
& \hat{D}(t)=2 \mu_{\mathrm{f}}\left\|\varepsilon\left(\hat{\boldsymbol{u}}_{\mathrm{f}}\right)\right\|_{L^{2}\left(\Omega_{\mathrm{f}}(t)\right)}^{2}+\eta_{\mathrm{s}}\left\|\hat{\boldsymbol{u}}_{\mathrm{s}}\right\|_{\mathcal{E}_{l}^{s}}^{2}+\frac{\hat{\pi}^{2}}{R_{\mathrm{d}}}+R_{\mathrm{p}} \hat{Q}^{2},
\end{aligned}
$$

and the symbol "*" denoting the measurement domain in consideration.

\subsubsection{Observer based on solid displacement measurements}

In practice, displacement-like measurements are more common [52] and, taking into account the time sampling of the measurements in medical imaging, we cannot afford to time-differentiate them to generate velocity measurements without unduly amplifying the noise. Therefore, we directly apply an observer of the form (10) which uses the $H^{\frac{1}{2}}\left(\Sigma_{0}\right)$ or $H^{1}\left(\Omega_{\mathrm{m}}\right)$ discrepancy measure as proposed in $[52,16]$ with the Schur Displacement Feedback (SDF) filter. Note that in this filter of the form (11) the control operator $\gamma \mathcal{H}^{\prime}$ does not appear as a force applying on the mechanical system, but instead modifies the velocity to displacement time-derivative identity. In fact the discrepancy measure $(8 \mathrm{a})$ or $(8 \mathrm{~b})$ has as corresponding tangent operator

$$
\begin{array}{ll}
\forall \boldsymbol{d}_{\mathrm{s}} \in \mathcal{V}_{s}^{d}, \quad \mathcal{P}_{H^{1}\left(\Omega_{\mathrm{m}}\right)}\{x, z\}(t)\left(\boldsymbol{d}_{\mathrm{s}}\right)=\left\langle\operatorname{Ext}_{\Omega_{\mathrm{m}}}^{\mathrm{s}}\left(\boldsymbol{z}_{d}-\left.\hat{\boldsymbol{y}}_{\mathrm{s}}\right|_{\Omega_{\mathrm{m}}}\right), \operatorname{Ext}_{\Omega_{\mathrm{m}}}^{\mathrm{s}}\left(\left.\boldsymbol{d}_{\mathrm{s}}\right|_{\Omega_{\mathrm{m}}}\right)\right\rangle_{\mathcal{E}_{l}^{s}}, \\
\forall \boldsymbol{d}_{\mathrm{s}} \in \mathcal{V}_{s}^{d}, \quad \mathcal{P}_{H^{\frac{1}{2}}\left(\Sigma_{0}\right)}\{x, z\}(t)\left(\boldsymbol{d}_{\mathrm{s}}\right)=\left\langle\operatorname{Ext}_{\Sigma_{0}}^{\mathrm{s}}\left(\boldsymbol{z}_{d}-\left.\hat{\boldsymbol{y}}_{\mathrm{s}}\right|_{\Omega_{\mathrm{m}}}\right), \operatorname{Ext}_{\Sigma_{0}}^{\mathrm{s}}\left(\left.\boldsymbol{d}_{\mathrm{s}}\right|_{\Omega_{\mathrm{m}}}\right)\right\rangle_{\mathcal{E}_{l}^{s}},
\end{array}
$$

that, for instance for $(15 \mathrm{~b})$, can be shown to be equivalent to

$$
\forall \boldsymbol{d}_{\mathrm{s}} \in \mathcal{V}_{s}^{d}, \quad \mathcal{P}_{H^{\frac{1}{2}}\left(\Sigma_{0}\right)}\{x, z\}(t)\left(\boldsymbol{d}_{\mathrm{s}}\right)=\left\langle\operatorname{Ext}_{\Sigma_{0}}^{\mathrm{s}}\left(\boldsymbol{z}_{d}-\left.\hat{\boldsymbol{y}}_{\mathrm{s}}\right|_{\Sigma_{0}}\right), \boldsymbol{d}_{\mathrm{s}}\right\rangle_{\mathcal{E}_{l}^{s}},
$$

using the extension characterization

$$
\forall \boldsymbol{d}_{\mathrm{s}} \text { such that }\left.\boldsymbol{d}_{\mathrm{s}}\right|_{\Sigma_{0}}=0, \quad\left\langle\operatorname{Ext}_{\Sigma_{0}}^{\mathrm{s}}\left(\boldsymbol{z}_{d}-\left.\hat{\boldsymbol{y}}_{\mathrm{s}}\right|_{\Sigma_{0}}\right), \boldsymbol{d}_{\mathrm{s}}\right\rangle_{\mathcal{E}_{l}^{s}}=0
$$

Similarly for (15a), we also have

$$
\forall \boldsymbol{d}_{\mathrm{s}} \in \mathcal{V}_{s}^{d}, \quad \mathcal{P}_{H^{1}(\omega)}\{x, z\}(t)\left(\boldsymbol{d}_{\mathrm{s}}\right)=\left\langle\operatorname{Ext}_{\Omega_{\mathrm{m}}}^{\mathrm{s}}\left(\boldsymbol{z}_{d}-\left.\hat{\boldsymbol{y}}_{\mathrm{s}}\right|_{\Omega_{\mathrm{m}}}\right), \boldsymbol{d}_{\mathrm{s}}\right\rangle_{\mathcal{E}_{l}^{s}} .
$$

The corresponding observer consists in modifying in System (1) the solid formulation (1c) into

$$
\left\{\begin{array}{rr}
\partial_{t} \hat{\boldsymbol{y}}_{\mathrm{s}}=\hat{\boldsymbol{u}}_{s}+\gamma_{d} \mathrm{Ext}_{*}^{\mathrm{s}}\left(\boldsymbol{z}_{d}-\left.\hat{\boldsymbol{y}}_{\mathrm{s}}\right|_{*}\right), & \text { in } \Omega_{0}^{\mathrm{s}} \\
\rho_{\mathrm{s}} \partial_{t} \hat{\boldsymbol{u}}_{\mathrm{s}}-\eta_{\mathrm{s}} \boldsymbol{\nabla} \cdot \boldsymbol{\sigma}_{\mathrm{s}}\left(\hat{\boldsymbol{u}}_{\mathrm{s}}\right)-\boldsymbol{\nabla} \cdot \boldsymbol{\sigma}_{\mathrm{s}}\left(\hat{\boldsymbol{y}}_{\mathrm{s}}\right)=\mathbf{0}, & \text { in } \Omega_{0}^{\mathrm{s}} \\
\hat{\boldsymbol{y}}_{\mathrm{s}}=\mathbf{0}, & \text { on } \Gamma_{0}^{\mathrm{d}} \\
\eta_{\mathrm{s}} \boldsymbol{\sigma}_{\mathrm{s}}\left(\hat{\boldsymbol{u}}_{\mathrm{s}}\right) \cdot \boldsymbol{n}_{\mathrm{s}}+\boldsymbol{\sigma}_{\mathrm{s}}\left(\hat{\boldsymbol{y}}_{\mathrm{s}}\right) \cdot \boldsymbol{n}_{\mathrm{s}}=\mathbf{0}, & \text { on } \Gamma_{0}^{\mathrm{n}}
\end{array}\right.
$$

with $\mathrm{Ext}_{*}^{\mathrm{s}}$ corresponding to $\mathrm{Ext}_{\Omega_{\mathrm{m}}}^{\mathrm{s}}$ or $\mathrm{Ext}_{\Sigma_{0}}^{\mathrm{s}}$ depending on the type of observations, and $\gamma_{d}$ the corresponding gain for the SDF. Note that such a correction can be considered for the observer $\hat{x}$ because we are only interested in numerical simulations (we could obviously not perform the same operation to control a genuine mechanical system in real life). 
Energetic aspects for the observer. The dissipative character of the SDF filter can be highlighted by an energy argument, as in Proposition 1. The first key point lies in the treatment of the term $\left\langle\hat{\boldsymbol{y}}_{\mathrm{s}}, \hat{\boldsymbol{u}}_{\mathrm{s}}\right\rangle_{\mathcal{E}_{1}^{s}}$ from $(18)_{2}$, which becomes non-standard due to the perturbed displacementvelocity relation $(18)_{1}$. We proceed as in [52], by evaluating the $\langle\cdot, \cdot\rangle_{\mathcal{E}_{l}^{s}}$-inner-product of $(18)_{1}$ with $\boldsymbol{y}_{\mathrm{s}}$. This yields the identity

$$
\left\langle\hat{\boldsymbol{u}}_{\mathrm{s}}, \hat{\boldsymbol{y}}_{\mathrm{s}}\right\rangle_{\mathcal{E}_{l}^{s}}=\left\langle\partial_{t} \hat{\boldsymbol{y}}_{\mathrm{s}}, \hat{\boldsymbol{y}}_{\mathrm{s}}\right\rangle_{\mathcal{E}_{l}^{s}}+\gamma_{d}\left\langle\operatorname{Ext}_{*}^{\mathrm{s}}\left(\hat{\boldsymbol{y}}_{\mathrm{s}}-\boldsymbol{z}_{d}\right), \hat{\boldsymbol{y}}_{\mathrm{s}}\right\rangle_{\mathcal{E}_{l}^{s}}
$$

Hence, from (16) and (17), we infer that

$$
\begin{aligned}
\left\langle\hat{\boldsymbol{u}}_{\mathrm{s}}, \hat{\boldsymbol{y}}_{\mathrm{s}}\right\rangle_{\mathcal{E}_{l}^{s}} & =\left\langle\partial_{t} \hat{\boldsymbol{y}}_{\mathrm{s}}, \hat{\boldsymbol{y}}_{\mathrm{s}}\right\rangle_{\mathcal{E}_{l}^{s}}+\gamma_{d}\left\langle\operatorname{Ext}_{*}^{\mathrm{s}}\left(\hat{\boldsymbol{y}}_{\mathrm{s}}-\boldsymbol{z}_{d}\right), \operatorname{Ext}_{*}^{\mathrm{s}}\left(\hat{\boldsymbol{y}}_{\mathrm{s}}\right)\right\rangle_{\mathcal{E}_{l}^{s}} \\
& \geq \frac{1}{2} \frac{\mathrm{d}}{\mathrm{d} t}\left\|\hat{\boldsymbol{y}}_{\mathrm{s}}\right\|_{\mathcal{E}_{l}^{s}}^{2}+\frac{\gamma_{d}}{2}\left\|\operatorname{Ext}_{*}^{\mathrm{s}}\left(\hat{\boldsymbol{y}}_{\mathrm{s}}\right)\right\|_{\mathcal{E}_{l}^{s}}^{2}-\frac{\gamma_{d}}{2}\left\|\operatorname{Ext}_{*}^{\mathrm{s}}\left(\boldsymbol{z}_{d}\right)\right\|_{\mathcal{E}_{l}^{s}}^{2} .
\end{aligned}
$$

Then, it should be noted that the modified velocity-displacement relation in the solid (18) 1 may affect the energy balance in the fluid. Indeed, since in general $\left.\partial_{t} \hat{\boldsymbol{y}}_{\mathrm{s}}\right|_{\Sigma_{0}} \neq\left.\hat{\boldsymbol{u}}_{s}\right|_{\Sigma_{0}}$, from the geometric and kinematic relations $(1 \mathrm{~d})_{1}$ we infer that the SDF filter induces a transpiration of the fluid across the interface, i.e., $\hat{\boldsymbol{u}}_{\mathrm{f}} \cdot \boldsymbol{n}_{\mathrm{f}} \neq \hat{\boldsymbol{w}} \cdot \boldsymbol{n}_{\mathrm{f}}$ on $\Sigma(t)$. In return, the convective term in (1a) 1 yields an artificial power in the global energy estimate. Fortunately, this can be fixed with ease by introducing a slight modification in the kinetic coupling, for instance, we suggest to replace (1d) by

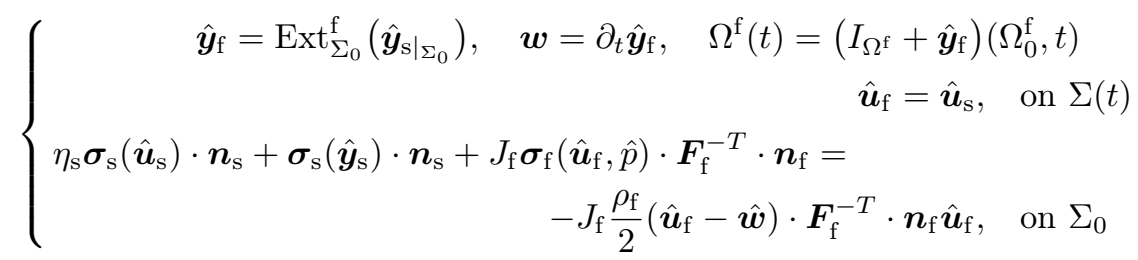

The last term in $(19)_{3}$ cancels the aforementioned artificial convective power. This term is nonzero when the SDF is active, since $\hat{\boldsymbol{u}}_{\mathrm{f}}$ is obtained from $\hat{\boldsymbol{u}}_{\mathrm{s}}$ while $\hat{\boldsymbol{w}}$ is obtained from the derivative of $\hat{\boldsymbol{y}}_{\mathrm{s}}$. When the standard relationship between $\boldsymbol{u}_{\mathrm{s}}$ and $\boldsymbol{y}_{\mathrm{s}}$ holds, this additional term vanishes. It can be therefore be viewed as "strongly consistent", in the sense that it vanishes as soon as the computed and measured displacements match. The above mentioned transpiration effects are also consistent in that sense.

In summary, for the coupled systems (1a), (1b), (18), (19), the identity (2) becomes

$$
\frac{\mathrm{d}}{\mathrm{d} t} \hat{E}(t) \leq-\hat{D}(t)-\frac{\gamma_{d}}{2}\left\|\operatorname{Ext}_{*}^{\mathrm{s}}\left(\hat{\boldsymbol{y}}_{\mathrm{s}}\right)\right\|_{\mathcal{E}_{l}^{s}}^{2}+\frac{\gamma_{d}}{2}\left\|\operatorname{Ext}_{*}^{\mathrm{s}}\left(\boldsymbol{z}_{d}\right)\right\|_{\mathcal{E}_{l}^{s}}^{2}-\int_{\Gamma^{\text {in }} \cup \Gamma^{\text {out }}} \frac{\rho_{\mathrm{f}}}{2} \hat{\boldsymbol{u}}_{\mathrm{f}} \cdot \boldsymbol{n}_{\mathrm{f}}\left|\hat{\boldsymbol{u}}_{\mathrm{f}}\right|^{2} d \Gamma .
$$

Owing to the stability properties of the extension operator $\mathrm{Ext}_{*}^{\mathrm{s}}$, the third term in the right-hand side of (20) can be controlled by $\frac{\gamma_{d}}{2}\left\|\boldsymbol{z}_{d}\right\|_{H^{1}\left(\Omega_{\mathrm{m}}\right)}^{2}$ or by $\frac{\gamma_{d}}{2}\left\|\boldsymbol{z}_{d}\right\|_{H^{\frac{1}{2}\left(\Sigma_{0}\right)}}^{2}$, depending on the choice of the tangent operator (15a) or (15b). Such energy estimates are often a valuable tool for the well-posedness analysis of this type of coupled problems (see, e.g., [47] for a recent review).

\section{REMARK 3 (MOTION OF THE FLUID DOMAIN)}

As already highlighted by the energy analysis, the modification of the relationship between the velocity and the displacement in the solid has to be carefully considered for the interaction with the fluid. In particular, it is desirable to preserve the kinematic compatibility at the fluidstructure interface, so we move $\Omega^{\mathrm{f}}(t)$ with an extension of the Lagrangian displacements of the solid $(19)_{1}$, and not from a displacement recomputed from the solid velocity. 


\subsection{First numerical experiments}

In order to have a first insight into the behavior and efficiency of the DVF and SDF observers in FSI, we now present two fluid-structure numerical experiments. The first one is purely illustrative, and the second one is representative of blood flows in large arteries. In Section 3, the estimators will be analyzed in more detail to better understand the different results presented in this section, and numerical illustrations will be provided using the same solid geometry and model, coupled with various simplified fluid flow models selected in accordance with the effect considered in the analysis. Furthermore, these exact same examples will also be used in the discussion section in order to assess alternative observer strategies.

All the physical quantities will be given in cgs units. In both experiments, we consider a straight tube of length 10 with diameter 1.7 and thickness 0.2 (see Figure 5). The solid density is $\rho_{\mathrm{s}}=1.2$, Young's modulus is $E=3.10^{5}$, the Poisson ratio is $\nu=0.46$, and the viscoelastic coefficient is $\eta_{\mathrm{s}}=10^{-3}$. Fluid viscosity and density are respectively $\mu_{\mathrm{f}}=0.035, \rho_{\mathrm{f}}=1$.

For the discretization, we apply a staggered solution to compute the fluid-structure problem, allowing to keep structure and fluid solvers independent. For the structure, we choose a Newmark scheme, whereas for the fluid we employ a semi-implicit coupling algorithm based on a Chorin-Temam projection scheme [29]. The viscous effects and the geometrical and convective nonlinearities are treated explicitly, and the pressure step is coupled implicitly to the solid. For the space discretization, we use conforming finite element triangulations in the fluid and solid domains, with first-order piecewise polynomials for all fluid and solid variables. Inf-sup compatibility in the fluid is circumvented via the natural pressure stabilization provided by the Chorin-Temam projection scheme, under the inverse parabolic-CFL constraint $h^{2} \lesssim \Delta t$ between the space, $h$, and time, $\Delta t$, discretization parameters. Dominant convective effects are stabilized by the SUPG method [12]. At last, we point out that we carefully adjust the solid constitutive parameters to allow sufficient compressibility to avoid numerical locking.

\subsubsection{Example 1 - Stabilization at rest configuration}

In the first experiment, the tube wall is clamped at the inlet and the outlet, a zero velocity is enforced at the inlet of the fluid and a zero traction is enforced at the outlet. At $t=0$, a displacement is imposed to the solid - taken from Example 2 at maximum deformation - hence

$$
\boldsymbol{y}_{\mathrm{s}}(0) \neq 0, \boldsymbol{u}_{\mathrm{s}}(0)=0,\left.\boldsymbol{y}_{\mathrm{f}}\right|_{\Sigma_{0}}(0)=\left.\boldsymbol{y}_{\mathrm{s}}\right|_{\Sigma_{0}}(0), \boldsymbol{w}_{\mathrm{f}}(0)=\boldsymbol{u}_{\mathrm{f}}(0)=0 .
$$

Since no external forces are applied, the system goes naturally back to equilibrium (fluid and solid at rest) due to the physical dissipation. Figure 2 shows the deformed domain at $t=0$ and the time response of the radial displacement at a point of the wall.

The purpose of this test is to compare the efficiency of the DVF and the SDF at accelerating this convergence to equilibrium. The measurements are therefore assumed to be zero for the velocity and displacement at the fluid-structure interface.

The results are summarized in Figure 3 and in Figure 4 for the error $\tilde{x}=x^{\text {ref }}-\hat{x}$ in the energy norm. In order to have a better insight into the effect of the feedbacks, we also plot the different components of the energy separately, namely, elastic and kinematic in the solid and kinematic in the fluid.

Interestingly, it appears that the SDF works much better than the DVF, whereas both have comparable efficacies for pure solid experiments [52]. Indeed, even if the SDF has been designed to have performances similar to the DVF for pure solid cases (see [52] and Section 3.1), it does generally not outperform it. This fact is directly related to the interaction of the solid with the fluid. In Section 3, we will provide an explanation, and a way to significantly improve the performance of the DVF for this problem. 

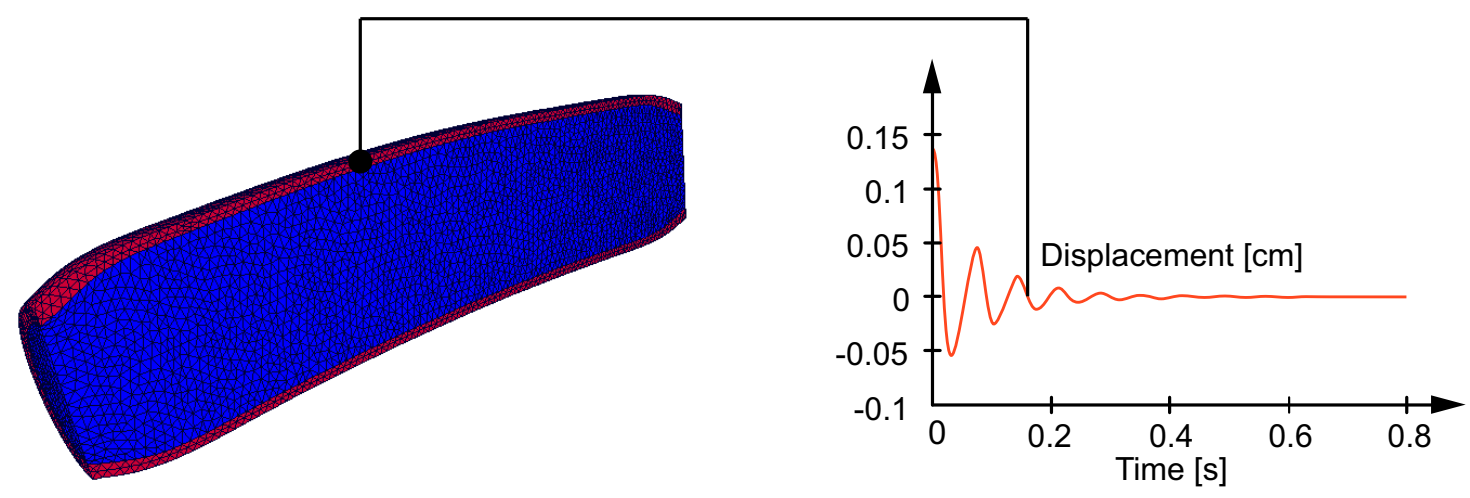

Figure 2: Initial deformed domain (left), displacements amplified 5 times, and time response of the displacement of one node of the wall (right).

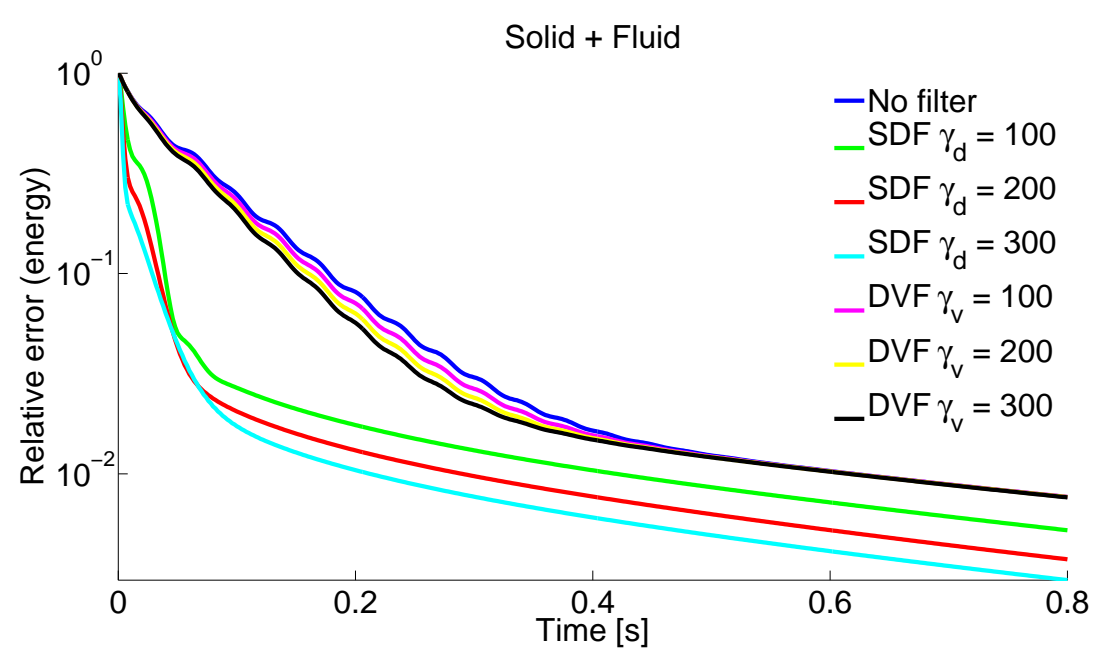

Figure 3: Decay of the total error in the energy norm for Example 1 (all curves are normalized with the initial energy) 

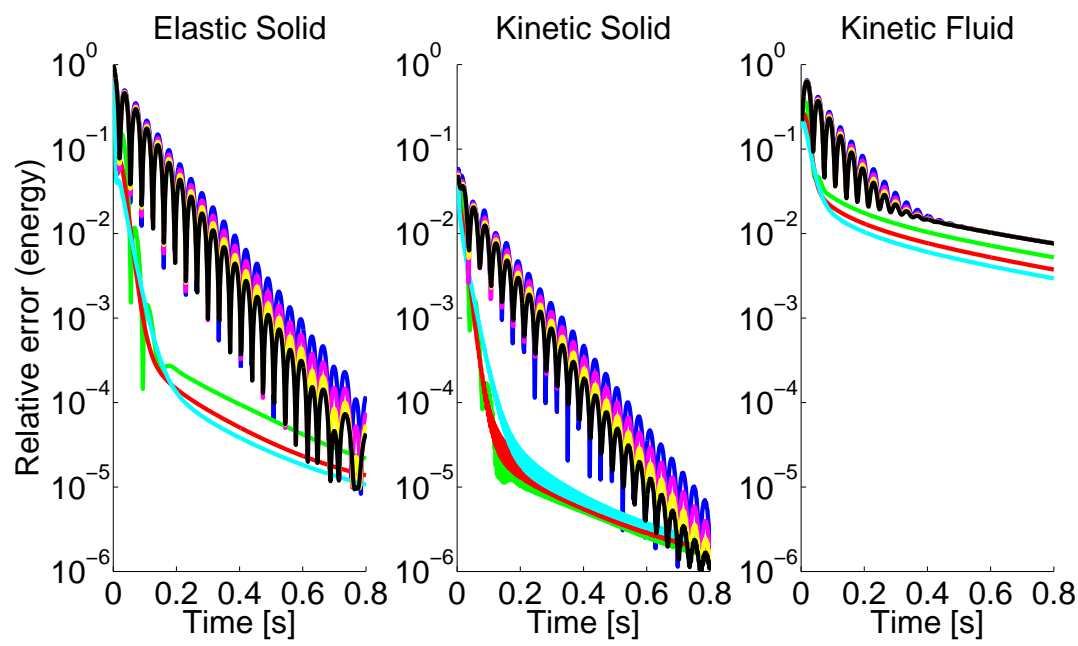

Figure 4: Decay of the error for solid and fluid in the energy norm for Example 1 (all curves are normalized with the initial energy). The colors correspond to the legend of Figure 3

\subsubsection{Example 2 - Tube with pulsatile flow}

In the second experiment, a time varying parabolic velocity profile is prescribed at the inlet with a peak velocity of about 110, see Figure 5 . At the outlet, the pressure $\bar{p}$ is assumed to be given by the Windkessel model in (1b) with $R_{p}=400, R_{d}=6.2 \cdot 10^{3}$ and $C=2.72 \cdot 10^{-4}$.

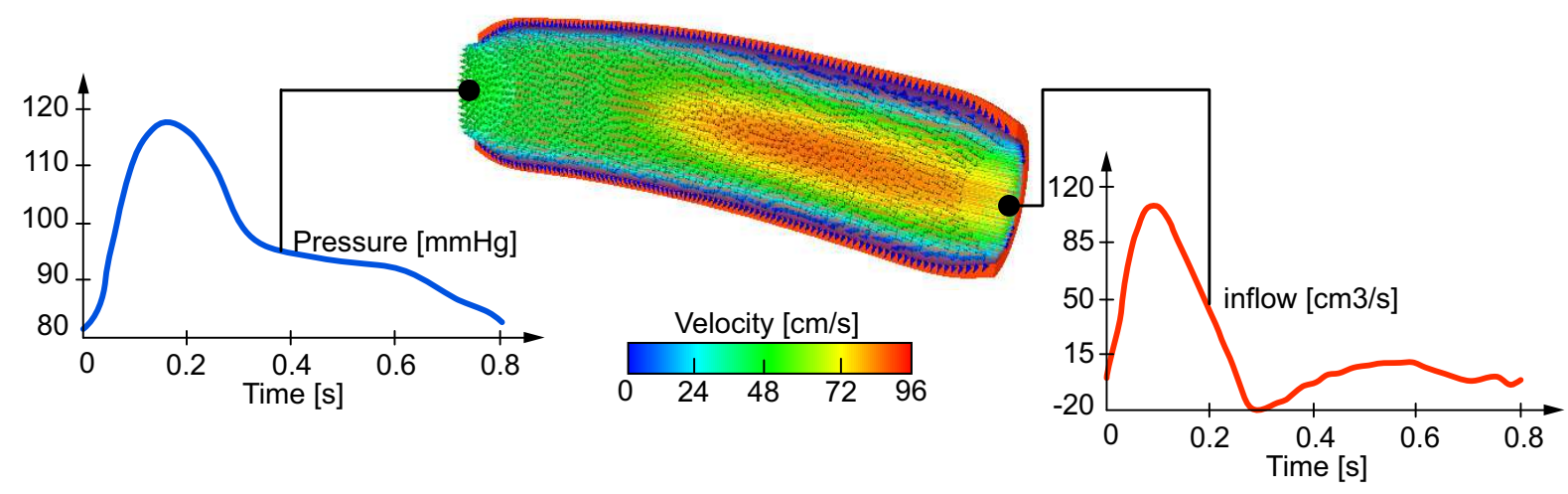

Figure 5: Velocity field and inflow condition for Example 2

The reference simulation used to generate the synthetic measurements is set as follows. At $t=0$, the pressure is constant and equal to $p(0)=80 \mathrm{mmHg}$, whereas all the other state variables are zero. During the whole simulation, the stress received by the structure is corrected by the initial one. Doing so, the solid only "feels" the difference with the initial phase. This is a simple way to account for the prestress in linear elastodynamics. We refer to [53], and references therein, for a discussion about prestress computation in a more general framework. 
To test the filters, we proceed as in the reference simulation until $t=0.8$ except that a perturbation is introduced in the Windkessel pressure $\bar{p}$ at $t=0$, i.e., $\bar{p}(0)=70 \mathrm{mmHg}$. Then a filter is applied for the second fluid-structure cycle $(0.8 \leq t \leq 1.6)$. Doing so, the filter acts on a system for which all state variables are perturbed with respect to the reference simulation (see Figure 6).
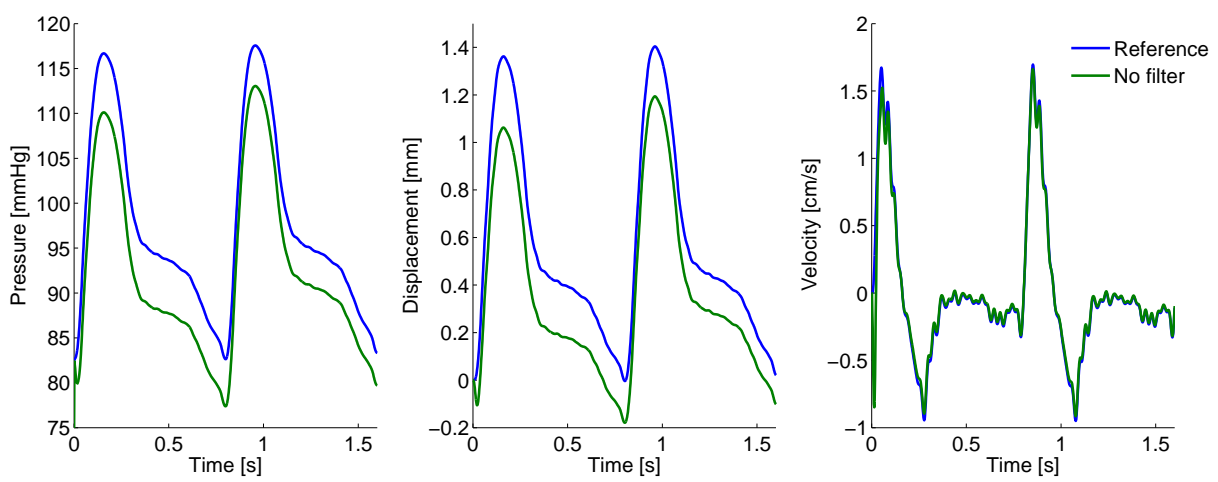

Figure 6: Construction of the error in the initial condition for Example 2. Value of the pressure, displacement, and velocity halfway through the vessel

The efficiency of the Luenberger filters are compared using the energy norm of the error (including the Windkessel energy). The results are reported in Figure 7 and Figure 8. As in the first experiment, the SDF outperforms the DVF. It is interesting to note that for the DVF and the non-filtered case, the decay rate is governed by the Windkessel time scale $R_{d} C \approx 1.7$. The negligible impact of the DVF on the Windkessel dynamics will be further illustrated in Section 3. With the SDF filter, at the very beginning the displacement correction increases the solid velocity error which propagates to the fluid velocity (see the solid and fluid kinetic energies at $t=0.8$ in Figure 8). But after a few milliseconds, this perturbation is quickly stabilized by the SDF filter. From about $t=1.2 \mathrm{~s}$, the global error is dominated by the fluid error which reaches a plateau, whereas the error in the solid and the Windkessel keeps decreasing. Again, we will propose an explanation of these observations in Section 3.

\section{REMARK 4 (EFFECT OF WHITE NOISE)}

We now briefly comment on the effect of noise in the estimation results. Consider for example the SDF, with a Gaussian noise added to synthetic measurements. The amount of noise is chosen as a certain percentage of the maximum displacement of the reference simulation, denoted by $\chi$ in the figure legend. The results corresponding to two levels of noise are plotted in Figure 9, together with the quantity

$$
\left\langle\operatorname{Ext}_{*}^{\mathrm{s}}(\chi), \operatorname{Ext}_{*}^{\mathrm{s}}(\chi)\right\rangle_{\mathcal{E}_{l}^{s}}^{\frac{1}{2}}
$$

which represents the energy norm of the extension of the noise in the solid domain. Note that Gaussian noise is generated independently for each degree of freedom, which explains the dramatic amplification effect observed in the elastic energy norm. In particular, it should be pointed out that for the large noise level considered the measurement error has a norm comparable to the reference solution itself, due to the high frequency contents of this noise. In practice, actual measurement errors would not feature such drastically high frequency components, hence this idealized modeling of the noise should be seen as a "worst case scenario". Nevertheless, the SDF 


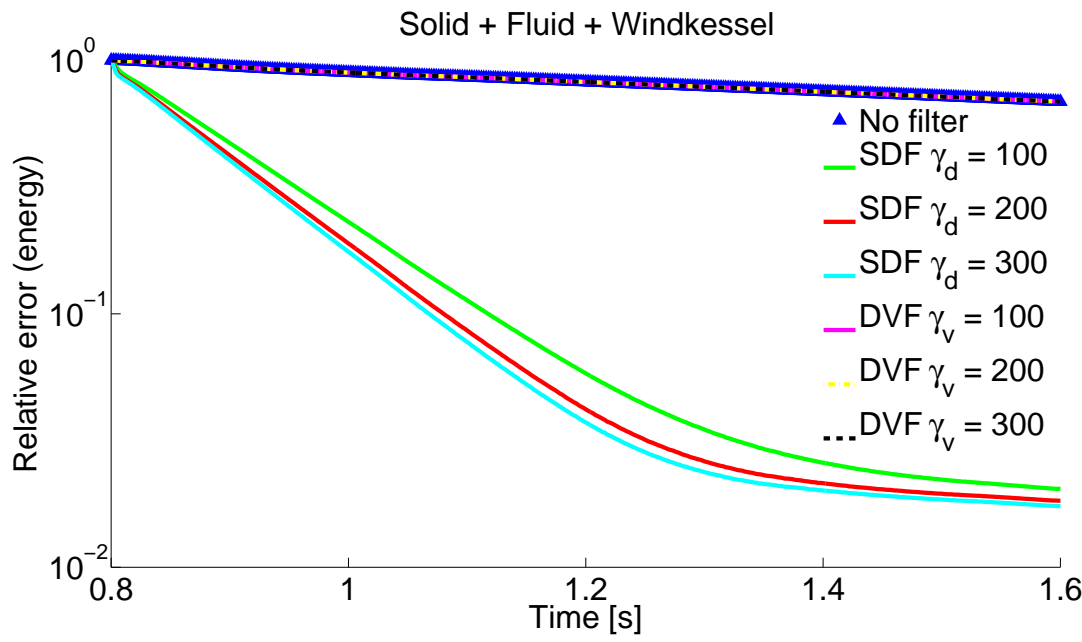

Figure 7: Decay of the total error in the energy norm for Example 2 (all curves are normalized with the initial energy)

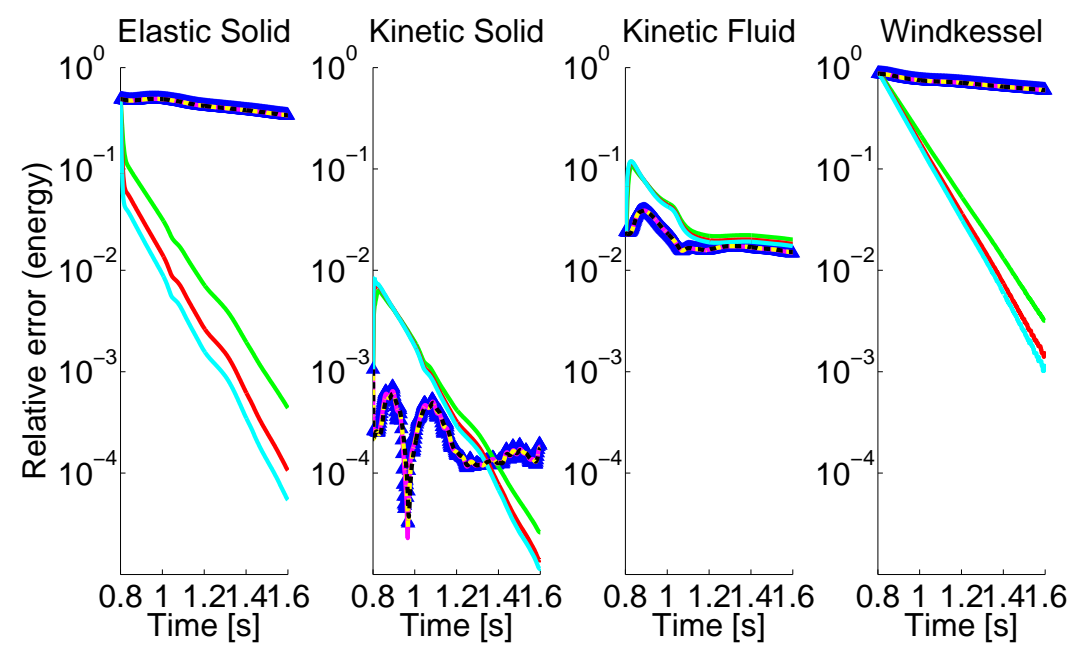

Figure 8: Decay of the error for solid and fluid in the energy norm for Example 2 (all curves are normalized with the initial energy). The colors correspond to the legend of Figure 7 
is still capable of effectively reducing the error much below the noise level, by a factor close to 10 in both cases (measured in energy).

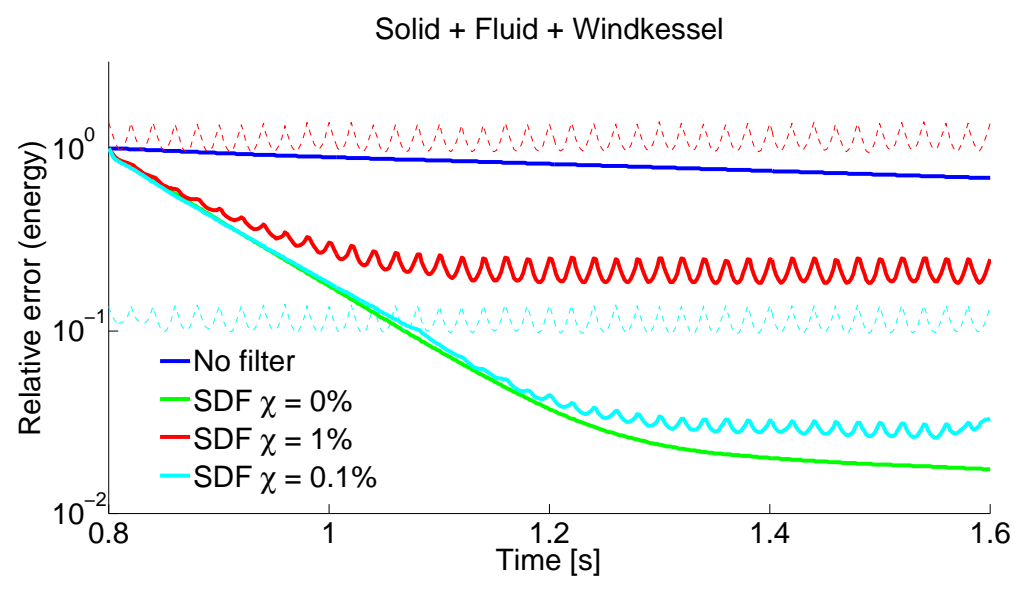

Figure 9: Effect of noise on the total error decay in Example 2 for the SDF with $\gamma_{d}=300$. The dashed lines correspond to Formula (21)

\section{Analysis of the estimators}

As said above, the purpose of the estimator (11) is to converge in time to the system (3), meaning that the error system denoted by

$$
\tilde{x}=x^{\text {ref }}-\hat{x}
$$

should stabilize to 0. For an FSI problem involving nonlinear formulations for the fluid and for the structure, the error system is a non-autonomous nonlinear system and the stabilization is therefore a largely open problem. As a matter of fact, one of the very few results pertaining to the stabilization of non-linear FSI problems is achieved in [59] with very specific feedback terms which could be employed for studying the observation error for a reference system at equilibrium - which is of course not the case for blood flows simulations.

As presented in [19], a sufficient condition for "local stability" - i.e. stability for small uncertainties around the trajectory $\left(\|\zeta\| \ll\left\|x_{0}\right\|\right.$ in (3)) - of the error can be proven by studying the linearization of the error system in 0 , i.e. a linearization of the observer around the real trajectory. Then, the difficulty of studying the stabilization of systems with time-dependent coefficients remains, since the reference itself is time-dependent. Note that a possible significant simplification would consist in studying the linearized formulation for the FSI problem assuming small displacements for the structure, no advection in the fluid and a fixed fluid domain. Therefore, the error system would consist of a stabilized linear coupled system whose convergence to 0 could be studied. This type of problem has been investigated in the literature, for example in [62] where a generic heat model is coupled to an elastic model.

Even for this linearized situation the complete analysis of the estimator remains largely open, both qualitatively - in terms of exponential stability - and even more quantitatively when 
trying to investigate the convergence time constant which purports to be much lower than the simulation time. For example the stabilization in the very recent result [37] is assessed with a full dissipation on the solid, whereas here we only assume partial observations, hence partial stabilization. Therefore, we undertake a numerical study of the characteristics of our estimator to assess its performance. As is classically done in FSI stability analysis - see e.g. [54] - we will compute the poles of the linearized system, albeit here for the error equations [52]. At least, this strategy will allow to distinguish good estimator candidates from dead end strategies.

We denote by capital letters the discretized state and operators of our FSI problem, and since we use standard Galerkin discretizations we still have access to the underlying variational formulation at the discrete level. We can then compute the poles of the eigenproblem

$$
(A-\Xi H) \widetilde{X}=\lambda \widetilde{X}
$$

associated with the dynamics of the error $\tilde{X}=X-\widehat{X}$. The operator $\Xi$ discretizing $\gamma H^{\prime}$ will be formalized in each case to adequately transcript the sense of the adjoint. The eigenvalue will help us quantify the decay rate - with slowest decay for highest real part. Hence, for reducing the error by a factor $\beta$ in a time $T_{\mathrm{c}}$, the feedback term should be designed so that

$$
\max \{\Re(\lambda)\} \leq \frac{\ln \beta}{T_{\mathrm{c}}} .
$$

Note that this involves the computation of the rightmost eigenvalues of (22) which are not necessarily the eigenvalues of smallest modulus. To fix the ideas in our context, an excellent performance would correspond to an attenuation of the initial condition error by a factor 10 within a time of $0.1 s$ (for a cardiac cycle of $1 s$ ) which gives about -25 as a reference target for $\max \{\Re(\lambda)\}$.

\subsection{Pure elastodynamics}

We start our investigation by recalling some results on a pure elastodynamics system. We consider a structure loaded with a given time-dependent pressure at its interface.

$$
\begin{cases}\partial_{t} \boldsymbol{y}_{\mathrm{s}}=\boldsymbol{u}_{\mathrm{s}}, & \text { in } \Omega_{0}^{\mathrm{s}} \\ \rho_{\mathrm{s}} \partial_{t} \boldsymbol{u}_{\mathrm{s}}-\eta_{\mathrm{s}} \boldsymbol{\nabla} \cdot \boldsymbol{\sigma}_{\mathrm{s}}\left(\boldsymbol{u}_{\mathrm{s}}\right)-\boldsymbol{\nabla} \cdot \boldsymbol{\sigma}_{\mathrm{s}}\left(\boldsymbol{y}_{\mathrm{s}}\right)=\mathbf{0}, & \text { in } \Omega_{0}^{\mathrm{s}} \\ \boldsymbol{y}_{\mathrm{s}}=\mathbf{0}, & \text { on } \Gamma_{0}^{\mathrm{d}} \\ \eta_{\mathrm{s}} \boldsymbol{\sigma}_{\mathrm{s}}\left(\boldsymbol{u}_{\mathrm{s}}\right) \cdot \boldsymbol{n}_{\mathrm{s}}+\boldsymbol{\sigma}_{\mathrm{s}}\left(\boldsymbol{y}_{\mathrm{s}}\right) \cdot \boldsymbol{n}_{\mathrm{s}}=\mathbf{0}, & \text { on } \Gamma_{0}^{\mathrm{n}} \\ \eta_{\mathrm{s}} \boldsymbol{\sigma}_{\mathrm{s}}\left(\boldsymbol{u}_{\mathrm{s}}\right) \cdot \boldsymbol{n}_{\mathrm{s}}+\boldsymbol{\sigma}_{\mathrm{s}}\left(\boldsymbol{y}_{\mathrm{s}}\right) \cdot \boldsymbol{n}_{\mathrm{s}}=-p \boldsymbol{n}_{\mathrm{s}}, & \text { on } \Sigma_{0}\end{cases}
$$

with the same notation as in Equation (1c).

We define the finite element discretization of (24) on the finite dimensional subspace $\mathcal{V}_{s, h} \subset \mathcal{V}_{s}$ and obtain the finite dimensional dynamical system in state space form

$$
\left[\begin{array}{cc}
K_{\mathrm{s}} & 0 \\
0 & M_{\mathrm{s}}
\end{array}\right]\left[\begin{array}{c}
\dot{Y}_{\mathrm{s}} \\
\dot{U}_{\mathrm{s}}
\end{array}\right]=\left[\begin{array}{cc}
0 & K_{\mathrm{s}} \\
-K_{\mathrm{s}} & -C_{\mathrm{s}}
\end{array}\right]\left[\begin{array}{c}
Y_{\mathrm{s}} \\
U_{\mathrm{s}}
\end{array}\right]+\left[\begin{array}{c}
0 \\
F
\end{array}\right]
$$


where the mass matrix $M_{\mathrm{s}}$, stiffness matrix $K_{\mathrm{s}}$ and $F$ are defined by

$$
\begin{aligned}
\forall \boldsymbol{u}_{\mathrm{s}}, \boldsymbol{v}_{\mathrm{s}} \in \mathcal{V}_{s, h}, \quad V_{\mathrm{s}}^{\boldsymbol{\top}} M_{\mathrm{s}} U_{\mathrm{s}}=\int_{\Omega_{0}^{\mathrm{s}}} \rho_{\mathrm{s}} \boldsymbol{u}_{\mathrm{s}} \cdot \boldsymbol{v}_{\mathrm{s}} d \Omega \\
\forall \boldsymbol{y}_{\mathrm{s}}, \boldsymbol{v}_{\mathrm{s}} \in \mathcal{V}_{s, h}, \quad V_{\mathrm{s}}^{\boldsymbol{\top}} K_{\mathrm{s}} Y_{\mathrm{s}}=\int_{\Omega_{0}^{\mathrm{s}}} \boldsymbol{\sigma}_{\mathrm{s}}\left(\boldsymbol{y}_{\mathrm{s}}\right): \varepsilon\left(\boldsymbol{v}_{\mathrm{s}}\right) d \Omega \\
\forall \boldsymbol{v}_{\mathrm{s}} \in \mathcal{V}_{s, h}, \quad V_{\mathrm{s}}^{\boldsymbol{\top}} F=\int_{\Sigma_{0}} p \boldsymbol{v}_{\mathrm{s}} \cdot \boldsymbol{n}_{\mathrm{s}} d \Gamma
\end{aligned}
$$

and $C_{\mathrm{s}}=\eta_{\mathrm{s}} K_{\mathrm{s}}$. We used $K_{\mathrm{s}}$ in the identity written in the first line of System (25) in order to emphasize the norm associated with the displacement field.

\subsubsection{Case of solid velocity measurements}

For this model, assuming that some velocity measurements are available, we can define a DVF state estimator

$$
\begin{cases}\partial_{t} \hat{\boldsymbol{y}}_{\mathrm{s}}=\hat{\boldsymbol{u}}_{s} & \text { in } \Omega_{0}^{\mathrm{s}} \\ \rho_{\mathrm{s}} \partial_{t} \hat{\boldsymbol{u}}_{\mathrm{s}}-\eta_{\mathrm{s}} \boldsymbol{\nabla} \cdot \boldsymbol{\sigma}_{\mathrm{s}}\left(\hat{\boldsymbol{u}}_{\mathrm{s}}\right)-\boldsymbol{\nabla} \cdot \boldsymbol{\sigma}_{\mathrm{s}}\left(\hat{\boldsymbol{y}}_{\mathrm{s}}\right)=\gamma_{v} \mathbb{1}_{*}\left(\boldsymbol{z}_{v}-\left.\hat{\boldsymbol{u}}_{\mathrm{s}}\right|_{*}\right), & \text { in } \Omega_{0}^{\mathrm{s}} \\ \hat{\boldsymbol{y}}_{\mathrm{s}}=\mathbf{0}, & \text { on } \Gamma^{\mathrm{d}} \\ \eta_{\mathrm{s}} \boldsymbol{\sigma}_{\mathrm{s}}\left(\hat{\boldsymbol{u}}_{\mathrm{s}}\right) \cdot \boldsymbol{n}_{\mathrm{s}}+\boldsymbol{\sigma}_{\mathrm{s}}\left(\hat{\boldsymbol{y}}_{\mathrm{s}}\right) \cdot \boldsymbol{n}_{\mathrm{s}}=\mathbf{0}, & \text { on } \Gamma_{0}^{\mathrm{n}} \\ \eta_{\mathrm{s}} \boldsymbol{\sigma}_{\mathrm{s}}\left(\hat{\boldsymbol{u}}_{\mathrm{s}}\right) \cdot \boldsymbol{n}_{\mathrm{s}}+\boldsymbol{\sigma}_{\mathrm{s}}\left(\hat{\boldsymbol{y}}_{\mathrm{s}}\right) \cdot \boldsymbol{n}_{\mathrm{s}}=-p \boldsymbol{n}_{\mathrm{s}}, & \text { on } \Sigma_{0}\end{cases}
$$

discretized into

$$
\left[\begin{array}{cc}
K_{\mathrm{s}} & 0 \\
0 & M_{\mathrm{s}}
\end{array}\right]\left[\begin{array}{c}
\dot{\hat{Y}}_{\mathrm{s}} \\
\dot{\hat{U}}_{\mathrm{s}}
\end{array}\right]=\left[\begin{array}{cc}
0 & K_{\mathrm{s}} \\
-K_{\mathrm{s}} & -C_{\mathrm{s}}-\gamma_{v} H_{v}^{\top} M_{*} H_{v}
\end{array}\right]\left[\begin{array}{c}
\hat{Y}_{\mathrm{s}} \\
\hat{U}_{\mathrm{s}}
\end{array}\right]+\left[\begin{array}{c}
0 \\
F+\gamma_{v} H_{v}^{\top} M_{*} Z
\end{array}\right]
$$

where $H_{v}$ is the discretization of the observation operator in $\mathcal{V}_{s, h}^{v}$ and $M_{*}$ is the matrix associated with the $L^{2}$-norm on the measurement domain. For instance, considering measurements on the interface, we can decompose the vector of degrees of freedom $U_{\mathrm{s}}$ into the degrees of freedom of $\Sigma_{0}$ and the internal degrees of freedom. Then we have

$$
\forall U_{\mathrm{s}}, \quad H_{v} U_{\mathrm{s}}=\left[\begin{array}{ll}
\mathbb{1} & 0
\end{array}\right]\left[\begin{array}{c}
U_{\mathrm{s}, \Sigma_{0}} \\
U_{\mathrm{s}, \mathrm{I}}
\end{array}\right]=U_{\mathrm{s}, \Sigma_{0}}
$$

and get

$$
\forall \boldsymbol{u}_{\mathrm{s}}, \boldsymbol{v}_{\mathrm{s}} \in \mathcal{V}_{s, h}, \quad V_{\mathrm{s}}^{\top} H_{v}^{\top} M_{\Sigma_{0}} H_{v} U_{\mathrm{s}}=V_{\mathrm{s}, \Sigma_{0}}^{\top} M_{\Sigma_{0}} U_{\mathrm{s}, \Sigma_{0}}=\int_{\Sigma_{0}} \boldsymbol{u}_{\mathrm{s}} \cdot \boldsymbol{v}_{\mathrm{s}} d \Gamma .
$$

Note that if the observations are undersampled in space, $H_{v}$ can be seen as a projector $\Pi_{\mathcal{Z}}$ on the subspace where the observation are defined and

$$
\forall \boldsymbol{u}_{\mathrm{s}}, \boldsymbol{v}_{\mathrm{s}} \in \mathcal{V}_{s, h}, \quad V_{\mathrm{s}}^{\boldsymbol{\top}} H_{v}^{\top} M_{\Sigma_{0}} H_{v} U_{\mathrm{s}}=\int_{\Sigma_{0}} \Pi_{\mathcal{Z}}\left(\boldsymbol{u}_{\mathrm{s}}\right) \cdot \Pi_{\mathcal{Z}}\left(\boldsymbol{v}_{\mathrm{s}}\right) d \Gamma
$$

From (22), we deduce the associated eigenvalue problem:

$$
\text { Find }\left(\Phi_{d}, \Phi_{v}, \lambda\right) \text { such that }\left[\begin{array}{cc}
0 & K_{\mathrm{s}} \\
-K_{\mathrm{s}} & -C_{\mathrm{s}}-\gamma_{v} H_{v}^{\top} M_{*} H_{v}
\end{array}\right]\left[\begin{array}{l}
\Phi_{d} \\
\Phi_{v}
\end{array}\right]=\lambda\left[\begin{array}{cc}
K_{\mathrm{s}} & 0 \\
0 & M_{\mathrm{s}}
\end{array}\right]\left[\begin{array}{l}
\Phi_{d} \\
\Phi_{v}
\end{array}\right] \text {. }
$$


Spectral sensitivity analysis. For the DVF, when eliminating $\Phi_{v}$ in (28), the computation of the poles can be performed by solving the following quadratic eigenvalue problem typical of the underlying second order PDE

$$
\text { Find }\left(\Phi_{d}, \lambda\right) \text { such that }\left(\lambda^{2} M_{\mathrm{s}}+\lambda\left(C_{\mathrm{s}}+\gamma_{v} H_{v}^{\top} M_{*} H_{v}\right)+K_{\mathrm{s}}\right) \Phi_{d}=0 .
$$

The solution $\left(\lambda, \Phi_{d}\right)$ of $(29)$ is a function of $\gamma_{v}$ and we denote by $\left(\lambda_{\mid 0}, \Phi_{d \mid 0}\right)$ the solutions for $\gamma_{v}=0$. Recalling that $C_{s}=\eta_{s} K_{s}$, they verify

$$
K_{\mathrm{s}} \Phi_{d \mid 0}=\omega^{2} M_{\mathrm{s}} \Phi_{d \mid 0}, \text { and } \lambda_{\mid 0}^{2}+\eta_{s} \omega^{2} \lambda_{\mid 0}+\omega^{2}=0,
$$

and we can normalize them such that $\Phi_{d \mid 0}^{\top} M_{s} \Phi_{d \mid 0}=1$ (note that we can consider real-only eigenmodes in our case).

Therefore, if we differentiate (29) with respect to $\gamma_{v}$ and evaluate the resulting expression at $\gamma_{v}=0$, we obtain

$$
\left(\left.2 \lambda_{\mid 0} \frac{\partial \lambda}{\partial \gamma_{v}}\right|_{\gamma_{v}=0} M_{\mathrm{S}}+\lambda_{\mid 0} H_{v}^{\top} M_{*} H_{v}+\frac{\partial \lambda}{\partial \gamma_{v}} C_{s}\right) \Phi_{d \mid 0}+\left.\left(\lambda_{\mid 0}^{2} M_{\mathrm{S}}+\lambda_{\mid 0} C_{s}+K_{\mathrm{s}}\right) \frac{\partial \Phi_{d}}{\partial \gamma_{v}}\right|_{\gamma_{v}=0}=0
$$

Finally, left-multiplying by $\Phi_{d \mid 0}^{\top}$ and using that, by definition $\left(\lambda_{\mid 0}^{2} M_{\mathrm{s}}+\lambda_{\mid 0} C_{s}+K_{\mathrm{s}}\right) \Phi_{d \mid 0}=0$, we obtain

$$
\left.\left(2+\eta_{s} \frac{\omega^{2}}{\lambda_{\mid 0}}\right) \frac{\partial \lambda}{\partial \gamma_{v}}\right|_{\gamma_{v}=0}=-\Phi_{d \mid 0}^{\top}\left(H_{v}^{\top} M_{*} H_{v}\right) \Phi_{d \mid 0} .
$$

Then for initially undamped structure the initial eigenvalues on the imaginary axis $\lambda_{\mid 0}^{2}=-\omega^{2}$ are moved to the half plane of negative real parts by

$$
\left.\frac{\partial \lambda}{\partial \gamma_{v}}\right|_{\gamma_{v}=0}=-\frac{\Phi_{d \mid 0}^{\top}\left(H_{v}^{\top} M_{*} H_{v}\right) \Phi_{d \mid 0}}{2}=-\frac{1}{2} \frac{\left\|H_{v} \Phi_{d \mid 0}\right\|_{M_{*}}^{2}}{\left\|\Phi_{d \mid 0}\right\|_{M_{s}}^{2}}
$$

Furthermore, when considering complete observations, we have $H_{v}=\mathbb{1}$ and we can rescale $M_{*}=M_{\mathrm{s}}$ so that

$$
\left.\frac{\partial \lambda}{\partial \gamma_{v}}\right|_{\gamma_{v}=0}=-\frac{1}{2}
$$

which shows that the DVF has a uniform effect on the damping sensitivity of all the eigenmodes. When considering a more general (restricted) observation operator, the corresponding classical observability condition $[4,13]$

$$
\int_{0}^{T_{\mathrm{obs}}}\left\|H_{v} U_{\mathrm{s}}(t)\right\|_{M_{*}}^{2} d t \geq \alpha\left(\left\|Y_{\mathrm{s}}(0)\right\|_{K_{\mathrm{s}}}^{2}+\left\|U_{\mathrm{s}}(0)\right\|_{M_{\mathrm{s}}}^{2}\right)
$$

for some (fixed) observation time $T_{\text {obs }}$ and strictly positive constant $\alpha$, also entails a similar uniform sensitivity on the eigenmodes, i.e.

$$
\left.\frac{\partial \lambda}{\partial \gamma_{v}}\right|_{\gamma_{v}=0} \leq-\frac{\alpha}{2 T_{\mathrm{obs}}}
$$

Of course, this is only a sensitivity analysis, and it is well-known that - as also occurs with the viscosity parameter $\eta_{s}$ - taking large values of the gain parameter may lead to overdamping where some poles hit the real axis and then one of each pair slides towards the right, i.e. loses some stability, when further increasing the gain, see Section 3.1.3 below for some numerical illustrations. 


\subsubsection{Case of solid displacement measurements}

Assuming now that we have at our disposal some displacement measurements, we define the SDF state estimator

$$
\begin{cases}\partial_{t} \hat{\boldsymbol{y}}_{\mathrm{s}}=\hat{\boldsymbol{u}}_{s}+\gamma_{d} \mathrm{Ext}_{*}^{s}\left(\boldsymbol{z}_{d}-\left.\hat{\boldsymbol{y}}_{\mathrm{s}}\right|_{*}\right), & \text { in } \Omega_{0}^{\mathrm{s}} \\ \rho_{\mathrm{s}} \partial_{t} \hat{\boldsymbol{u}}_{\mathrm{s}}-\eta_{\mathrm{s}} \boldsymbol{\nabla} \cdot \boldsymbol{\sigma}_{\mathrm{s}}\left(\hat{\boldsymbol{u}}_{\mathrm{s}}\right)-\boldsymbol{\nabla} \cdot \boldsymbol{\sigma}_{\mathrm{s}}\left(\hat{\boldsymbol{y}}_{\mathrm{s}}\right)=\mathbf{0}, & \text { in } \Omega_{0}^{\mathrm{s}} \\ \hat{\boldsymbol{y}}_{\mathrm{s}}=\mathbf{0}, & \text { on } \Gamma^{\mathrm{d}} \\ \eta_{\mathrm{s}} \boldsymbol{\sigma}_{\mathrm{s}}\left(\hat{\boldsymbol{u}}_{\mathrm{s}}\right) \cdot \boldsymbol{n}_{\mathrm{s}}+\boldsymbol{\sigma}_{\mathrm{s}}\left(\hat{\boldsymbol{y}}_{\mathrm{s}}\right) \cdot \boldsymbol{n}_{\mathrm{s}}=\mathbf{0}, & \text { on } \Gamma_{0}^{\mathrm{n}} \\ \eta_{\mathrm{s}} \boldsymbol{\sigma}_{\mathrm{s}}\left(\hat{\boldsymbol{u}}_{\mathrm{s}}\right) \cdot \boldsymbol{n}_{\mathrm{s}}+\boldsymbol{\sigma}_{\mathrm{s}}\left(\hat{\boldsymbol{y}}_{\mathrm{s}}\right) \cdot \boldsymbol{n}_{\mathrm{s}}=-p \boldsymbol{n}_{\mathrm{s}}, & \text { on } \Sigma_{0}\end{cases}
$$

Following [52], we can compute the extension by a penalization method. We define the operator

$$
L_{\mathrm{s}, *}^{\epsilon}: Z_{d} \rightarrow \underset{X}{\operatorname{argmin}}\left\{\frac{1}{2} \epsilon X^{\top} K_{s} X+\frac{1}{2}\left\|Z_{d}-H_{d} X\right\|_{M_{*}}\right\}^{2}
$$

with $\epsilon$ a small parameter with respect to the inverse of the Young modulus of the structure, such that

$$
\forall Z_{d}, \quad L_{\mathrm{s}, *}^{\epsilon} Z_{d}=\left(\epsilon K_{\mathrm{s}}+H_{d}^{\top} M_{*} H_{d}\right)^{-1} H_{d}^{\top} M_{*} Z_{d}
$$

is a discretization of $\operatorname{Ext}_{*}^{\mathrm{s}}\left(\boldsymbol{z}_{d}\right)$. Thus we have

$$
\forall \boldsymbol{d}_{\mathrm{s}} \in \mathcal{V}_{\mathrm{s}, h}, \quad\left\langle\operatorname{Ext}_{*}^{\mathrm{s}}\left(\boldsymbol{z}_{d}-\left.\hat{\boldsymbol{y}}_{\mathrm{s}}\right|_{*}\right), \boldsymbol{d}_{\mathrm{s}}\right\rangle_{\mathcal{E}_{l}^{s}}=D_{\mathrm{s}}^{\top} K_{\mathrm{s}}\left(\epsilon K_{\mathrm{s}}+H_{d}^{\top} M_{*} H_{d}\right)^{-1} H_{d}^{\top} M_{*}\left(Z_{d}-H_{d} Y_{\mathrm{s}}\right),
$$

where $D_{\mathrm{s}}$ stands for the vector of degrees of freedom associated to $\boldsymbol{d}_{\mathrm{s}} \in \mathcal{V}_{\mathrm{s}, h}$. Therefore, the discretized system reads

$$
\left[\begin{array}{cc}
\left(\epsilon K_{\mathrm{s}}+H_{d}^{\top} M_{*} H_{d}\right) & 0 \\
0 & M_{\mathrm{s}}
\end{array}\right]\left[\begin{array}{c}
\dot{\hat{Y}}_{\mathrm{s}} \\
\dot{\hat{U}}_{\mathrm{s}}
\end{array}\right]=\left[\begin{array}{cc}
-\gamma_{d} H_{d}^{\top} M_{*} H_{d} & \left(\epsilon K_{\mathrm{s}}+H_{d}^{\top} M_{*} H_{d}\right) \\
-K_{\mathrm{s}} & -C_{\mathrm{s}}
\end{array}\right]\left[\begin{array}{c}
\hat{Y}_{\mathrm{s}} \\
\hat{U}_{\mathrm{s}}
\end{array}\right]+\left[\begin{array}{c}
\gamma_{d} H_{d}^{\top} M_{*} Z \\
F
\end{array}\right]
$$

and the associated eigenvalue problem becomes

Find $\left(\Phi_{d}, \Phi_{v}, \lambda\right)$ such that

$$
\left[\begin{array}{cc}
-\gamma_{d} H_{d}^{\top} M_{*} H_{d} & \left(\epsilon K_{\mathrm{s}}+H_{d}^{\top} M_{*} H_{d}\right) \\
-K_{\mathrm{s}} & -C_{\mathrm{s}}
\end{array}\right]\left[\begin{array}{l}
\Phi_{d} \\
\Phi_{v}
\end{array}\right]=\lambda\left[\begin{array}{cc}
\left(\epsilon K_{\mathrm{s}}+H_{d}^{\top} M_{*} H_{d}\right) & 0 \\
0 & M_{\mathrm{s}}
\end{array}\right]\left[\begin{array}{l}
\Phi_{d} \\
\Phi_{v}
\end{array}\right]
$$

Spectral sensitivity analysis. For the SDF, the eigenvalue problem can also be solved using a quadratic eigenvalue problem inferred from (35) which takes the form

Find $\left(\Phi_{d}, \lambda\right)$ such that $\left(\lambda^{2} M_{\mathrm{s}}+\lambda\left(C_{\mathrm{s}}+\gamma_{d} M_{\mathrm{s}} L_{\mathrm{s}, *}^{\epsilon} H_{d}\right)+K_{\mathrm{s}}+\gamma_{d} C_{\mathrm{s}} L_{\mathrm{s}, *}^{\epsilon} H_{d}\right) \Phi_{d}=0$,

and note that in presence of viscosity the SDF adds stiffness to the system. The solutions of (36) for $\gamma_{d}=0$, are still given by $\left(\lambda_{\mid 0}, \Phi_{d \mid 0}\right)$ as defined in the DVF case. Here, we verify that

$$
\left.\frac{\partial \lambda}{\partial \gamma_{d}}\right|_{\gamma_{d}=0}=-\frac{\Phi_{d \mid 0}^{\top} M_{\mathrm{s}} L_{\mathrm{s}, *}^{\epsilon} H_{d} \Phi_{d \mid 0}}{2}+O\left(\eta_{s}\right)
$$

such that if $\eta_{s}=0$ then the sensitivity of the poles with respect to the SDF gain is given by

$$
\left.\frac{\partial \lambda}{\partial \gamma_{d}}\right|_{\gamma_{d}=0}=-\frac{\Phi_{d \mid 0}^{\top} M_{\mathrm{s}} L_{\mathrm{s}, *}^{\epsilon} H_{d} \Phi_{d \mid 0}}{2}=-\frac{\Phi_{d \mid 0}^{\top} K_{\mathrm{s}} L_{\mathrm{s}, *}^{\epsilon} H_{d} \Phi_{d \mid 0}}{2 \omega^{2}},
$$


recalling that $K_{\mathrm{s}} \Phi_{d \mid 0}=\omega^{2} M_{\mathrm{s}} \Phi_{d \mid 0}$. Then, using the characterization of the operator $L_{\mathrm{s}, *}^{\epsilon}$ obtained in Appendix A, for any vector $D_{\mathrm{s}}$ associated with $\boldsymbol{d}_{\mathrm{s}} \in \mathcal{V}_{s, h}$ we have

$$
D_{\mathrm{s}}^{\top} K_{\mathrm{s}} L_{\mathrm{s}, *}^{\epsilon} H_{d} D_{\mathrm{s}}=D_{\mathrm{s}}^{\top} H_{d}^{\top}\left(L_{\mathrm{s}, *}^{\epsilon}\right)^{\top} K_{\mathrm{s}} L_{\mathrm{s}, *}^{\epsilon} H_{d} D_{\mathrm{s}}+\epsilon D_{\mathrm{s}}^{\top}\left(\Gamma_{\mathrm{s}, *}^{\epsilon}\right)^{\top} H_{d}^{\top} M_{*} H_{d} \Gamma_{\mathrm{s}, *}^{\epsilon} D_{\mathrm{s}}
$$

which allows to rewrite

$$
\left.\frac{\partial \lambda}{\partial \gamma_{d}}\right|_{\gamma_{d}=0}=-\frac{1}{2} \frac{\left\|L_{\mathrm{s}, *}^{\epsilon}\left(H_{d} \Phi_{d \mid 0}\right)\right\|_{K_{s}}^{2}}{\left\|\Phi_{d \mid 0}\right\|_{K_{s}}^{2}}-\frac{\epsilon}{2} \frac{\left\|H_{d} \Gamma_{\mathrm{s}, *}^{\epsilon} \Phi_{d \mid 0}\right\|_{M_{*}}^{2}}{\left\|\Phi_{d \mid 0}\right\|_{K_{s}}^{2}} .
$$

In this expression we retrieve the norm of the lifting of the observed part of a vector as it appeared in the continuous energy balance (20) up to a small term of order $\epsilon$ and adequate sign arising from the definition of the extension by penalization. Hence, when considering complete observations and the limit $\epsilon \rightarrow 0$, we have for the SDF in elastodynamics

$$
\left.\frac{\partial \lambda}{\partial \gamma_{d}}\right|_{\gamma_{d}=0}=-\frac{1}{2}
$$

as we had for the DVF. When considering a more general (restricted) observation operator, the corresponding observability condition becomes

$$
\int_{0}^{T_{\mathrm{obs}}}\left\|L_{\mathrm{s}, *}^{\epsilon}\left(H_{d} Y_{\mathrm{s}}\right)\right\|_{K_{s}}^{2} d t \geq \alpha\left(\left\|Y_{\mathrm{s}}(0)\right\|_{K_{\mathrm{s}}}^{2}+\left\|U_{\mathrm{s}}(0)\right\|_{M_{\mathrm{s}}}^{2}\right),
$$

for some (fixed) observation time $T_{\text {obs }}$ and strictly positive constant $\alpha$. This observability condition has been established for internal observations in the case of a scalar wave equation in [16]. If the condition is verified, then we again have a uniform sensitivity on the eigenmodes, i.e.

$$
\left.\frac{\partial \lambda}{\partial \gamma_{d}}\right|_{\gamma_{d}=0} \leq-\frac{\alpha}{2 T_{\mathrm{obs}}}
$$

\subsubsection{Spectral numerical experiments}

We now verify numerically the analytical observations of the previous paragraph by means of a spectral analysis. For the computations, we consider the geometry and parameters of Section 2.3. In Figure 10, we report the corresponding 10 poles of smallest modulus for different values of the gains $\gamma_{d}$ and $\gamma_{v}$.

As expected from the stability analysis of the previous paragraph, both filters have a similar behavior with respect to the gain when considering full observations. Moreover, we observe that the SDF adds some slight stiffness (namely, the term $\gamma_{d} C_{\mathrm{s}} L_{\mathrm{s}, *}^{\epsilon} H_{d}$ in (36)) due to the presence of damping in the system modeled by $C_{\mathrm{s}}$, particularly for the highest frequency poles. Note also that for large values of the gain some poles may travel to the right, especially after hitting the real axis. This phenomenon known as over-damping is classical in the stabilization of structural mechanics based systems, see e.g. [58].

\subsection{Added mass effect for elastodynamic coupled with potential flow}

We now investigate the impact of the fluid coupling on the filters performances, by first considering an inviscid incompressible fluid. Hence, the forcing pressure $p$ in Equation (24) is driven by the following elliptic problem

$$
\left\{\begin{aligned}
-\Delta p & =0, & & \text { in } \Omega_{0}^{\mathrm{f}} \\
\nabla p \cdot \boldsymbol{n}_{\mathrm{f}} & =0, & & \text { on } \Gamma^{\text {in }} \\
p & =0, & & \text { on } \Gamma^{\text {out }}
\end{aligned}\right.
$$



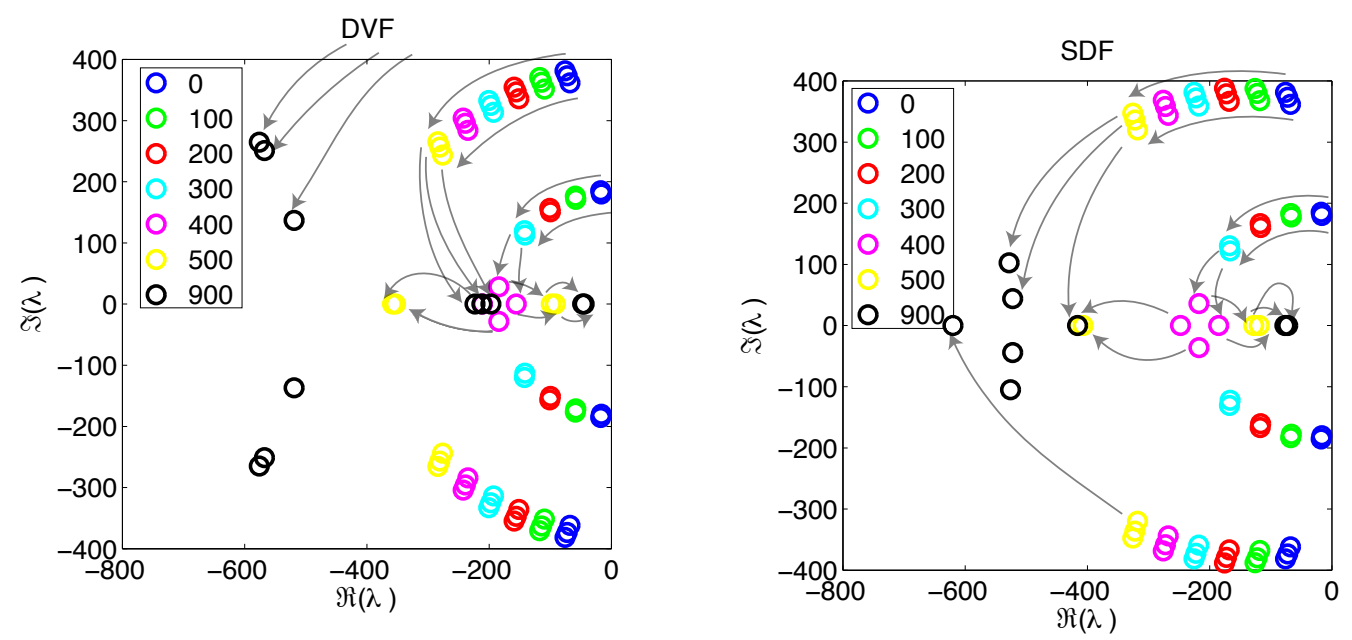

Figure 10: Poles of the DVF (left) and the SDF (right) estimators in elastodynamics with full observation and for different values of the gain. The grey arrows sketch the trajectories of the poles when increasing the feedback gain

with $\Omega_{0}^{\mathrm{f}}=\Omega_{\mathrm{f}}(0)$ and the interface coupling conditions reduce to

$$
\left\{\begin{aligned}
\nabla p \cdot \boldsymbol{n}_{\mathrm{f}}=-\rho_{\mathrm{f}} \partial_{t} \boldsymbol{u}_{\mathrm{s}} \cdot \boldsymbol{n}_{\mathrm{f}}, & \text { on } \Sigma_{0} \\
\eta_{\mathrm{s}} \boldsymbol{\sigma}_{\mathrm{s}}\left(\boldsymbol{u}_{\mathrm{s}}\right) \cdot \boldsymbol{n}_{\mathrm{s}}+\boldsymbol{\sigma}_{\mathrm{s}}\left(\boldsymbol{y}_{\mathrm{s}}\right) \cdot \boldsymbol{n}_{\mathrm{s}}-p \boldsymbol{n}_{\mathrm{f}}=\mathbf{0}, & \text { on } \Sigma_{0}
\end{aligned}\right.
$$

Although simplified, the coupled problem derived from (24)-(39)-(40) contains some of the main features involved in FSI in blood flows, particularly, the so-called added-mass effect (see e.g. [14]).

After discretization in space, we get the following system of ordinary differential equations,

$$
\left[\begin{array}{cc}
K_{\mathrm{s}} & 0 \\
0 & M_{\mathrm{s}}+M_{A}
\end{array}\right]\left[\begin{array}{c}
\dot{Y}_{\mathrm{s}} \\
\dot{U}_{\mathrm{s}}
\end{array}\right]=\left[\begin{array}{cc}
0 & K_{\mathrm{s}} \\
-K_{\mathrm{s}} & -C_{\mathrm{s}}
\end{array}\right]\left[\begin{array}{c}
Y_{\mathrm{s}} \\
U_{\mathrm{s}}
\end{array}\right]
$$

where $M_{A}$ denotes the discrete version of the added-mass operator, given by

$$
M_{A}=\rho_{\mathrm{f}} G K_{p}^{-1} G^{\top} .
$$

Here, $K_{p}$ and stand for the finite element matrices of the Laplacian and $G$ is associated with the boundary term

$$
\forall P \in \mathcal{V}_{p, h}, U_{s} \in \mathcal{V}_{s, h}, \quad U_{\mathrm{s}}^{\top} G P=\int_{\Sigma_{0}} p \boldsymbol{u}_{\mathrm{s}} \cdot \boldsymbol{n}_{\mathrm{f}} d \Gamma
$$

Then, we can straightforwardly repeat the analysis performed in Section 3.1, hence the eigenproblems associated with the DVF and SDF estimators are obtained from (28) and (35) by simply replacing $M_{\mathrm{s}}$ by $M_{\mathrm{s}}+M_{A}$ in the right-hand side.

\subsubsection{The added mass effect on stabilization efficiency}

We now investigate the impact of the added-mass operator $M_{A}$ on the effectiveness of the estimators, by analyzing the sensitivity of the right-most eigenvalues of these systems with respect 
to the filter gains $\gamma_{d}$ and $\gamma_{v}$. According to the previous paragraph, we can write the quadratic eigenvalue problems as

Find $\left(\Phi_{a, d}, \lambda_{a}\right)$ such that $\left(\lambda_{a}^{2}\left(M_{\mathrm{s}}+M_{A}\right)+\lambda_{a}\left(C_{\mathrm{s}}+\gamma_{v} H_{v}^{\top} M_{*} H_{v}\right)+K_{\mathrm{s}}\right) \Phi_{a, d}=0$,

for the DVF, and

$$
\left(\lambda_{a}^{2}\left(M_{\mathrm{s}}+M_{A}\right)+\lambda_{a}\left(C_{\mathrm{s}}+\gamma_{d}\left(M_{\mathrm{s}}+M_{A}\right) L_{\mathrm{s}, *}^{\epsilon} H_{d}\right)+K_{\mathrm{s}}+\gamma_{d} C_{\mathrm{s}} L_{\mathrm{s}, *}^{\epsilon} H_{d}\right) \Phi_{a, d}=0,
$$

for the SDF. Assuming again $C_{\mathrm{s}}=0$ and introducing, this time, the eigenmodes $\Phi_{a, d \mid 0}$ satisfying

$$
K_{\mathrm{s}} \Phi_{a, d \mid 0}=\omega_{a}^{2}\left(M_{\mathrm{s}}+M_{A}\right) \Phi_{a, d \mid 0}, \lambda_{a \mid 0}^{2}=-\omega_{a}^{2}, \Phi_{a, d \mid 0}^{\top}\left(M_{\mathrm{s}}+M_{A}\right) \Phi_{a, d \mid 0}=1,
$$

the sensitivities become for the DVF

$$
\left.\frac{\partial \lambda}{\partial \gamma_{v}}\right|_{\gamma_{v}=0}=-\frac{\Phi_{a, d \mid 0}^{\top}\left(H_{v}^{\top} M_{*} H_{v}\right) \Phi_{a, d \mid 0}}{2}=-\frac{1}{2} \frac{\left\|H_{v} \Phi_{a, d \mid 0}\right\|_{M_{*}}^{2}}{\left\|\Phi_{a, d \mid 0}\right\|_{M_{s}+M_{A}}^{2}} .
$$

Then, for the SDF we comparatively obtain

$$
\begin{aligned}
\left.\frac{\partial \lambda}{\partial \gamma_{d}}\right|_{\gamma_{d}=0} & =-\frac{\Phi_{a, d \mid 0}^{\top}\left(\left(M_{\mathrm{s}}+M_{A}\right) L_{\mathrm{s}, *}^{\epsilon} H_{d}\right) \Phi_{a, d \mid 0}}{2}=\frac{\Phi_{a, d \mid 0}^{\top}\left(K_{\mathrm{s}} L_{\mathrm{s}, *}^{\epsilon} H_{d}\right) \Phi_{a, d \mid 0}}{2 \omega_{a}^{2}} \\
& =-\frac{\Phi_{a, d \mid 0}^{\top} H_{d}^{\top}\left(L_{\mathrm{s}, *}^{\epsilon}\right)^{\top} K_{\mathrm{s}} L_{\mathrm{s}, *}^{\epsilon} H_{d} \Phi_{a, d \mid 0}}{2 \omega_{a}^{2}}=-\frac{1}{2} \frac{\left\|L_{\mathrm{s}, *}^{\epsilon}\left(H_{d} \Phi_{a, d \mid 0}\right)\right\|_{K_{s}}^{2}}{\left\|\Phi_{a, d \mid 0}\right\|_{K_{s}}^{2}} .
\end{aligned}
$$

Note that, by construction of the estimator, the DVF does not include the added-mass matrix in the expression of the sensitivity, while the SDF does. This is the reason why the SDF can outperform the DVF in FSI problems.

To illustrate this point, let us consider a case with complete observations. The sensitivities of the DVF applied to the simplified FSI problem become

$$
\left.\frac{\partial \lambda}{\partial \gamma_{v}}\right|_{\gamma_{v}=0}=-\frac{\Phi_{a, d \mid 0}^{\top} M_{\mathrm{s}} \Phi_{a, d \mid 0}}{2}=-\frac{1}{2}\left(1-\frac{\left\|\Phi_{a, d \mid 0}\right\|_{M_{A}}^{2}}{\left\|\Phi_{a, d \mid 0}\right\|_{M_{s}+M_{A}}^{2}}\right),
$$

to be compared with (31) in the pure elastodynamics case. For the problems of interest, for some modes $\Phi_{a, d \mid 0}^{\top} M_{A} \Phi_{a, d \mid 0}$ is close to 1 (see Table 1 ). Thus, the sensitivity is close to zero - recall that eigenmodes are normalized with respect to $M_{s}+M_{A}$ - which explains the poor behavior of the DVF in the FSI test case presented in Example 1 (Section 2.3.1). On the contrary, for the SDF applied to the simplified FSI problem, the sensitivity remains:

$$
\left.\frac{\partial \lambda}{\partial \gamma_{d}}\right|_{\gamma_{d}=0}=-\frac{1}{2}
$$

as in the pure elastodynamics case (38).

\subsubsection{Spectral numerical experiments}

The difference of performances can be also understood in Figure 11, where the poles of the DVF and the SDF are displayed for different values of gains. As shown in the sensitivity analysis, the SDF is capable to uniformly stabilize the poles. By contrast, the performance of the DVF depends on the added-mass contribution $\Phi_{a, d \mid 0}^{\top} M_{A} \Phi_{a, d \mid 0}$, whose values are shown in Table 1. In fact, the response in Example 1 (see Figure 3-right) is dominated by the first frequency $\left(\Im\left(\lambda_{\mid 0}\right)=98\right)$, which confirms the hypothesis of the impact of the added-mass in the performance of the DVF in this test case. 

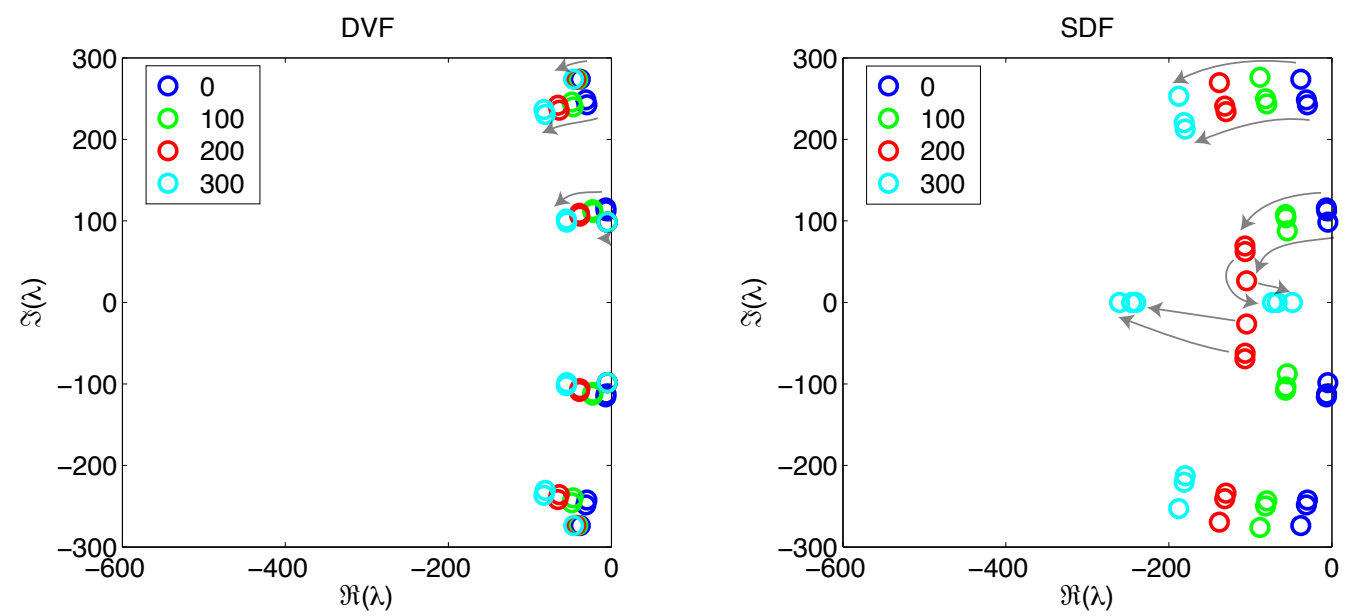

Figure 11: Poles of the DVF (left) and the SDF (right) estimators for the elastodynamics/pressure system, with full observation and for different values of the gain

\begin{tabular}{c|cccccc}
$\Phi_{a, d \mid 0}^{\top} M_{A} \Phi_{a, d \mid 0}$ & 0.99 & 0.61 & 0.61 & 0.59 & 0.58 & 0.93 \\
\hline frequency $\Im\left(\lambda_{a \mid 0}\right)$ & 98 & 112 & 116 & 244 & 250 & 276
\end{tabular}

Table 1: Values of coefficient $\Phi_{a, d \mid 0}^{\top} M_{A} \Phi_{a, d \mid 0}$ for the poles from Figure 11(left)

\subsubsection{The DVFam filter improvement for solid velocity measurements}

To improve the performance of the DVF in FSI problems, we propose to modify the scalar product used to define the adjoint $\mathcal{H}^{\prime}$ in (10). The DVF designed for elastodynamics problem was based on the standard $L^{2}$ scalar product. For FSI problems, we advocate to include the added-mass operator in the definition of the scalar product. This defines a new filter, that will be called DVFam. Whereas the matrix of the DVF was defined by $H_{v}^{\top} M_{*} H_{v}$, the matrix of the DVFam filter is given by:

$$
H_{v}^{\top}\left(M_{*}+H_{v} M_{A} H_{v}^{\top}\right) H_{v}
$$

Repeating mutatis mutandis the sensitivity analysis, and assuming complete observations, we can check that

$$
\left.\frac{\partial \lambda}{\partial \gamma_{v}}\right|_{\gamma_{v}=0}=-\frac{1}{2}
$$

with the DVFam. This is a clear improvement compared to (46). The spectral analysis reported in Figure 12 confirms this behavior. Note that in (49), the sensitivity does not depend on the range of the physical parameters, contrary to (46).

In practice, to avoid the calculation of the added-mass matrix, one can extend the filtered 


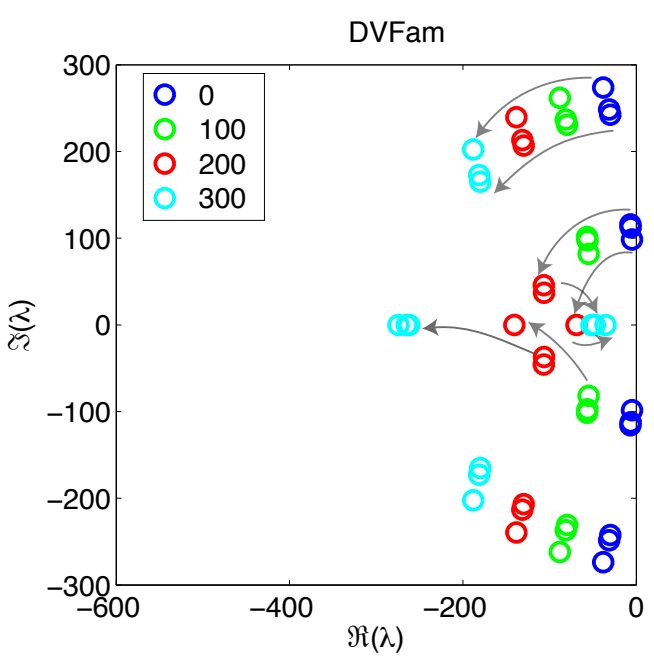

Figure 12: Performance of the DVFam filter (to be compared with Figure 11, left)

structural system as follows:

$$
\begin{aligned}
{\left[\begin{array}{ccc}
K_{\mathrm{s}} & 0 & 0 \\
0 & M_{\mathrm{s}} & 0 \\
0 & 0 & 0
\end{array}\right]\left[\begin{array}{c}
\dot{\hat{Y}}_{\mathrm{s}} \\
\dot{\hat{U}_{\mathrm{s}}} \\
\dot{\Lambda}
\end{array}\right] } \\
=\left[\begin{array}{ccc}
0 & K_{\mathrm{s}} & 0 \\
-K_{\mathrm{s}} & -C_{\mathrm{s}}-\gamma_{v} H_{v}^{\top} M_{*} H_{v} & \gamma_{v} \rho_{\mathrm{f}} H_{v}^{\top} H_{v} G \\
0 & -G^{\top} H_{v}^{\top} H_{v} & -K_{p}
\end{array}\right]\left[\begin{array}{c}
\widehat{Y}_{\mathrm{s}} \\
\widehat{U}_{\mathrm{s}} \\
\Lambda
\end{array}\right]+\left[\begin{array}{c}
0 \\
\gamma_{v} H_{v}^{\top} M_{*} Z+F \\
G^{\boldsymbol{\top}} H_{v}^{\top} Z
\end{array}\right],
\end{aligned}
$$

where $F$ denotes the forces coming from the fluid. A less expensive solution would be to use an approximation of the added-mass operator (for example using an algebraic approximation of the Dirichlet-to-Neumann operator as done in [48]) but this option will not be considered here.

To test this new filter in a nonlinear case with partial observations, we consider again Example 1. The results are presented in Figure 13. We observe that the performance of the DVFam filter is similar to the SDF, and much better than the DVF. Note that, for this test case, the addedmass term in the definition of the DVFam filter was not updated to account for the fluid domain deformation.

\subsection{Elastodynamics-pressure coupling with lumped-parameter model}

In hemodynamics, the Windkessel model plays an important role to define the outflow boundary conditions. To better understand its influence on the behavior of the filters, we consider the coupled fluid-structure problem (24)-(39)-(40) in which $p=\bar{p}$ on $\Gamma^{\text {out }}$, where $\bar{p}$ is the Windkessel proximal pressure. We then couple this system with the Windkessel distal pressure model $\pi$ from 


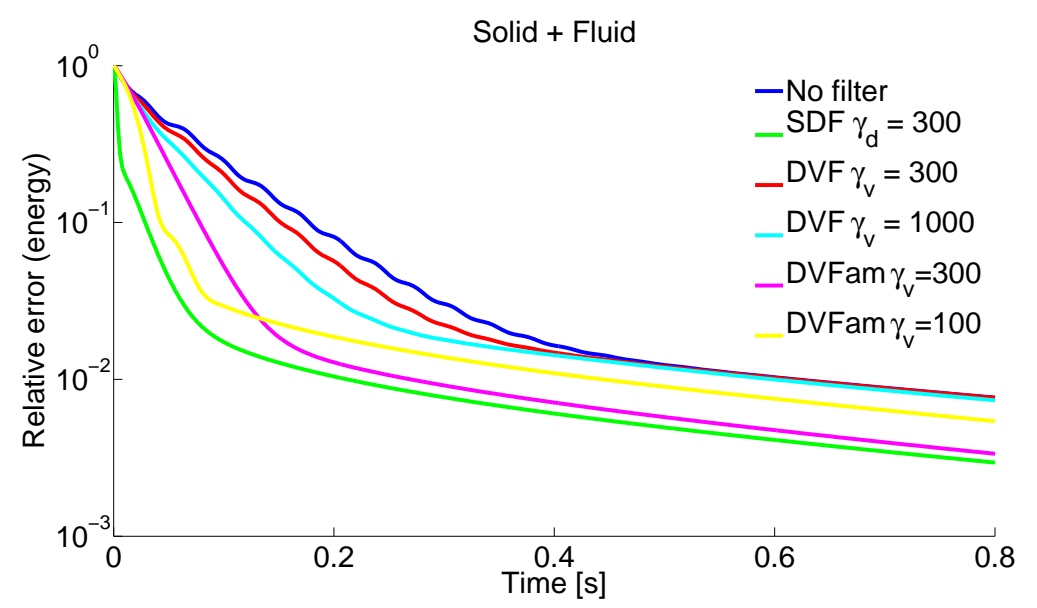

Figure 13: Comparison between DVFam, DVF and SDF in Example 1

equations (1b) where the outlet flux $Q$ can be obtained here from the structure velocity

$$
Q=-\int_{\Sigma_{0}} \boldsymbol{u}_{\mathrm{s}} \cdot \boldsymbol{n}_{\mathrm{f}} d \Gamma
$$

since there is no inflow on $\Gamma^{\text {in }}$ (recall $\left.(39)_{2}\right)$. After spatial discretization, there exists an operator $S$ such that

$$
Q=-S^{\top} U_{\mathrm{s}},
$$

and we verify that the boundary term $G$ defined in (42) verifies $S=-G(1 \ldots 1)^{\top}$. In the principle of virtual work, the additional virtual power associated with the new pressure condition $p=\bar{p}$ on $\Gamma^{\text {out }}$ is then given by

$$
\begin{aligned}
\forall \boldsymbol{v}_{s} \in \mathcal{V}_{s}, \quad \int_{\Sigma_{0}} \bar{p} \boldsymbol{v}_{\mathrm{s}} \cdot \boldsymbol{n}_{\mathrm{f}} d \Gamma & =\int_{\Sigma_{0}}\left(\pi+R_{p} Q\right) \boldsymbol{v}_{\mathrm{s}} \cdot \boldsymbol{n}_{\mathrm{f}} d \Gamma \\
& =\int_{\Sigma_{0}} \pi \boldsymbol{v}_{\mathrm{s}} \cdot \boldsymbol{n}_{\mathrm{f}} d \Gamma+R_{p} Q \int_{\Sigma_{0}} \boldsymbol{v}_{\mathrm{s}} \cdot \boldsymbol{n}_{\mathrm{f}} d \Gamma
\end{aligned}
$$

which discretizes into

$$
\forall v_{s} \in \mathcal{V}_{h, s}, \quad \int_{\Sigma_{0}} \bar{p} \boldsymbol{v}_{\mathrm{s}} \cdot \boldsymbol{n}_{\mathrm{f}} d \Gamma=V_{s}^{\top}\left(-R_{p} S \cdot S^{T} U_{s}+S \pi\right),
$$

leading to the spatial discretization

$$
\left[\begin{array}{ccc}
K_{\mathrm{s}} & 0 & 0 \\
0 & M_{\mathrm{s}}+M_{A} & 0 \\
0 & 0 & C
\end{array}\right]\left[\begin{array}{c}
\dot{Y}_{\mathrm{s}} \\
\dot{U}_{\mathrm{s}} \\
\dot{\pi}
\end{array}\right]=\left[\begin{array}{ccc}
0 & K_{\mathrm{s}} & 0 \\
-K_{\mathrm{s}} & -C_{\mathrm{s}}-R_{p} S \cdot S^{\boldsymbol{\top}} & S \\
0 & -S^{\boldsymbol{\top}} & -\frac{1}{R_{d}}
\end{array}\right]\left[\begin{array}{c}
Y_{\mathrm{s}} \\
U_{\mathrm{s}} \\
\pi
\end{array}\right]
$$

Note that the system (51) is clearly dissipative and the energy dissipation associated with the Windkessel is directly $\frac{\pi^{2}}{R_{d}}+R_{p} Q^{2}$ as in (2). 

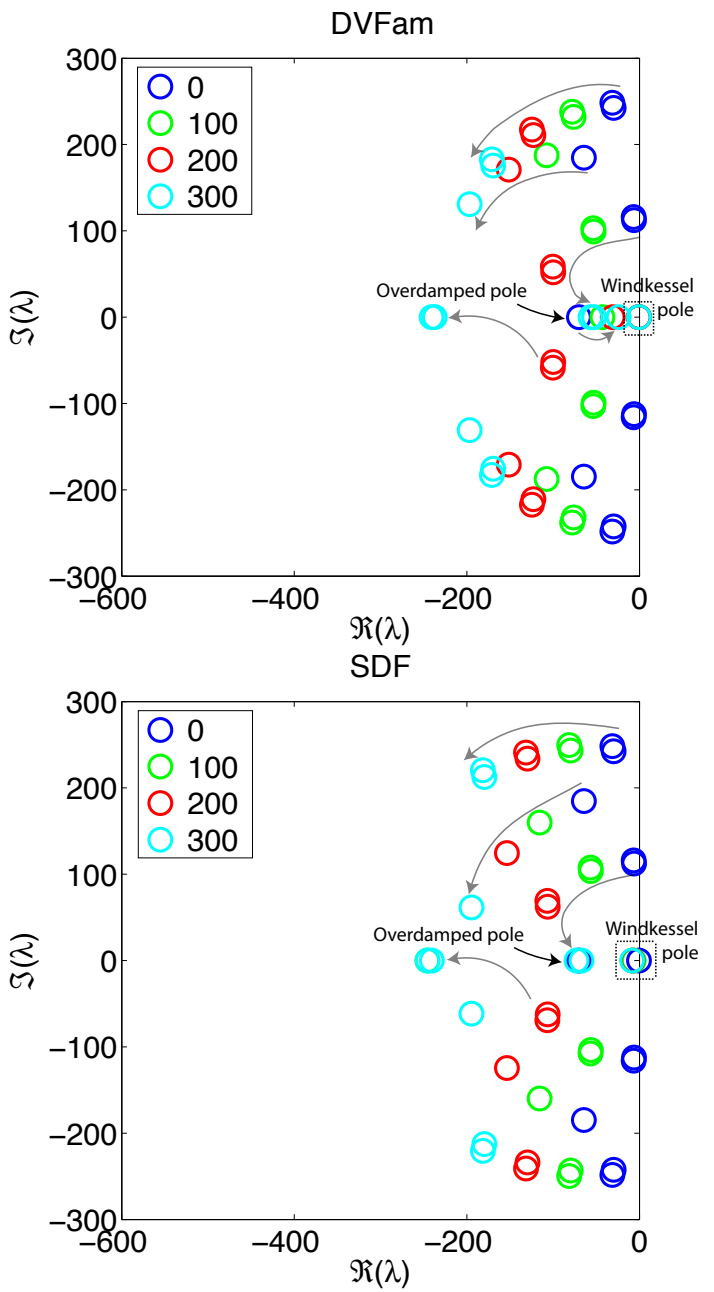

Figure 14: Poles of the DVFam (left) and the SDF (right) estimators (elastodynamics-pressure coupling with lumped-parameter model) with full observation and for different values of the gain. The pole marked as "overdamped" appears due to the damping introduced by the Windkessel resistances. The pole marked as "Windkessel" is that satifying Equation (56) 

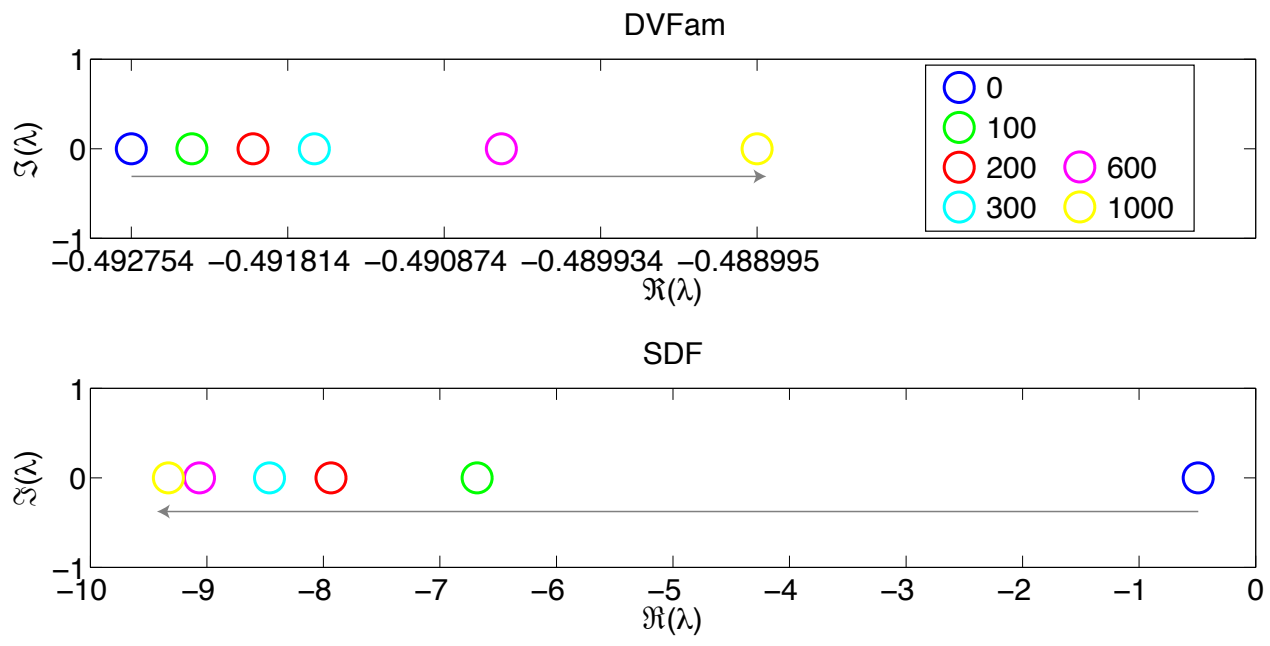

Figure 15: Zoomed views around the Windkessel pole when varying the filters gains

Spectral numerical investigations and analysis. Figure 14 shows the spectrum locus for the discrete DVFam and SDF estimators, computed by substituting the respective matrices in (51) according to (27) modified with (48) for the DVFam, and (34) for the SDF, see also Figure 15 for corresponding zoomed views around 0 . The values of $R_{\mathrm{p}}, R_{\mathrm{d}}$ and $C$ are taken as in Example 2. The novelty in this test case lies is the presence of the pole coming from the Windkessel model (i.e. the right-most one on the real axis in Figure 14). We observe that the DVFam has a negligible effect on this pole, unlike the SDF, which appears to quite effectively stabilize the error, including for this Windkessel-related pole. In order to obtain some insight into the very different behaviors of the two filters for this pole, let us write the complete modal equation satisfied by the corresponding (real) eigen-pair. First considering the non-filtered system, we consider $\left(\Phi_{w, d \mid 0}, \Phi_{w, v \mid 0}, \Phi_{w, v \mid 0}\right)$ and $\lambda_{w \mid 0}$ solution of

$$
\left\{\begin{array}{l}
K_{\mathrm{s}} \Phi_{w, v \mid 0}=\lambda_{w \mid 0} K_{\mathrm{s}} \Phi_{w, d \mid 0} \\
-K_{\mathrm{s}} \Phi_{w, d \mid 0}-\left(C_{\mathrm{s}}+R_{p} S \cdot S^{\top}\right) \Phi_{w, v \mid 0}+S \Phi_{w, \pi \mid 0}=\lambda_{w \mid 0}\left(M_{\mathrm{s}}+M_{A}\right) \Phi_{w, v \mid 0} \\
-S^{\top} \Phi_{w, v \mid 0}-\frac{1}{R_{d}} \Phi_{w, \pi \mid 0}=\lambda_{w \mid 0} C \Phi_{w, \pi \mid 0}
\end{array}\right.
$$

Considering a real eigenvector $\left(\Phi_{w, d \mid 0}^{\text {real }}, \Phi_{w, v \mid 0}^{\text {real }}, \Phi_{w, \pi \mid 0}^{\text {real }}\right)$ associated with a real negative eigenvalue $\lambda_{w \mid 0}^{\text {real }}$ and left-multiplying (52) by this eigenvector yields the "modal energy equation"

$$
\lambda_{w \mid 0}^{\mathrm{real}}=-\Phi_{w, v \mid 0}^{\mathrm{real}}{ }^{\top}\left(C_{\mathrm{S}}+R_{p} S \cdot S^{\top}\right) \Phi_{w, v \mid 0}^{\mathrm{real}}-\frac{1}{R_{d}}\left(\Phi_{w, \pi \mid 0}^{\mathrm{real}}\right)^{2}
$$

using the natural energy normalization $\left(\Phi_{w, d \mid 0}^{\text {real }}\right)^{\top} K_{\mathrm{s}} \Phi_{w, d \mid 0}^{\text {real }}+\Phi_{w, v \mid 0}^{\text {real }}{ }^{\top}\left(M_{\mathrm{s}}+M_{A}\right) \Phi_{w, v \mid 0}^{\text {real }}+C\left(\Phi_{w, \pi \mid 0}^{\text {real }}\right)^{2}=$

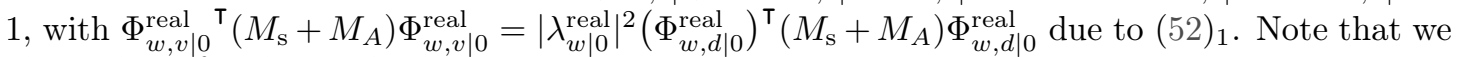
always have for the Rayleigh quotient

$$
\frac{\left(\Phi_{w, d \mid 0}^{\mathrm{real}}\right)^{\top} K_{\mathrm{s}} \Phi_{w, d \mid 0}^{\mathrm{real}}}{\left(\Phi_{w, d \mid 0}^{\mathrm{real}}\right)^{\top}\left(M_{\mathrm{s}}+M_{A}\right) \Phi_{w, d \mid 0}^{\mathrm{real}}} \geq\left(\omega_{a}^{\mathrm{min}}\right)^{2},
$$


where $\omega_{a}^{\min }$ denotes the fundamental natural frequency for the solid system with added mass (39)-(40) without damping, with $\omega_{a}^{\min }$ typically $O(100)$. Hence, for the Windkessel pole

$$
\frac{\Phi_{w, v \mid 0}^{\mathrm{real}}{ }^{\top}\left(M_{\mathrm{s}}+M_{A}\right) \Phi_{w, v \mid 0}^{\mathrm{real}}}{\left(\Phi_{w, d \mid 0}^{\mathrm{real}}\right)^{\top} K_{\mathrm{s}} \Phi_{w, d \mid 0}^{\mathrm{real}}} \leq\left(\frac{\left|\lambda_{w \mid 0}^{\mathrm{real}}\right|}{\omega_{a}^{\mathrm{min}}}\right)^{2} \ll 1,
$$

since $\lambda_{w \mid 0}^{\text {real }}=O(1)$ here, namely, kinetic energy is negligible compared to elastic energy. Therefore, in essence we have a "quasi-static" mode, characterized by dropping $\Phi_{w, v \mid 0}^{\text {real }}$ in $(52)_{2}$, namely,

$$
\Phi_{w, d \mid 0}^{\mathrm{real}} \approx\left(K_{\mathrm{s}}\right)^{-1} S \Phi_{w, \pi \mid 0}^{\mathrm{real}}
$$

which corresponds to the deformation produced by a constant pressure applied on the interface, and likewise from (53) and the normalization

$$
\lambda_{w \mid 0}^{\mathrm{real}} \approx-\frac{1}{R_{d}}\left(\Phi_{w, \pi \mid 0}^{\mathrm{real}}\right)^{2} \approx-\left[R_{d}\left(C+S^{\top}\left(K_{\mathrm{s}}\right)^{-1} S\right)\right]^{-1}
$$

For example, in our context we found $\lambda_{w \mid 0}^{\text {real }}=-0.4927$ and the approximation (56) gives -0.4937 . This shows that the isolated Windkessel time constant $R_{d} C$ may be increased by the static capacitance effect of the solid. Then the filter effect can be investigated by analysing the changes induced in (53), i.e.

$$
\lambda_{D V F}=-\Phi_{w, v}^{\mathrm{real}}\left(C_{\mathrm{s}}+R_{p} S \cdot S^{\top}+\gamma_{v} H_{v}^{\top} M_{*} H_{v}\right) \Phi_{w, v}^{\mathrm{real}}-\frac{1}{R_{d}}\left(\Phi_{w, \pi}\right)^{2},
$$

which does not produce any significant effect due to negligible velocity, whereas

$$
\lambda_{S D F}=-\gamma_{d} \Phi_{w, d}^{\mathrm{real} \top} K_{\mathrm{s}}\left(\epsilon K_{\mathrm{s}}+H_{d}^{\top} M_{*} H_{d}\right)^{-1} H_{d}^{\top} M_{*} H_{d} \Phi_{w, d}^{\mathrm{real}}-\Phi_{w, v}^{\mathrm{real}{ }^{\top}}\left(C_{\mathrm{s}}+R_{p} S \cdot S^{\top}\right) \Phi_{w, v}^{\mathrm{real}}-\frac{1}{R_{d}}\left(\Phi_{w, \pi}\right)^{2},
$$

where as already discussed $K_{\mathrm{s}}\left(\epsilon K_{\mathrm{s}}+H_{d}^{\top} M_{*} H_{d}\right)^{-1} H_{d}^{\top} M_{*} H_{d}$ is symmetric, and reduces to $K_{\mathrm{s}}$ in case of perfect observation. This shows that the SDF filter can be effective thanks to the finite elastic energy of the static mode. We refer to the appendix for a more detailed sensitivity analysis for both filters.

As a conclusion, in typical hemodynamics problems - such as that presented in Example 2 - the DVF is affected by two different issues: the added-mass effect and the Windkessel boundary conditions. While the first difficulty can be effectively treated by considering the modified DVFam strategy, the second one is intrinsic to the quasi-static nature of the Windkessel pole, which makes it impossible to stabilize with velocity-based strategies.

\section{REMARK 5 (WINDKESSEL OBSERVER)}

One way to further stabilize the pole resulting from the Windkessel coupling is to make use of additional measurements on $\bar{p}$ or $\pi$. Hence, we can formulate the observers

$$
R_{\mathrm{d}} C \dot{\hat{\pi}}+\hat{\pi}=R_{\mathrm{d}} \hat{Q}+\gamma_{\pi}\left(z_{\pi}-\hat{\pi}\right),
$$

and

$$
R_{\mathrm{d}} C \dot{\overline{\hat{p}}}+\hat{\bar{p}}=\left(R_{\mathrm{d}}+R_{\mathrm{p}}\right) \hat{Q}+R_{\mathrm{d}} C R_{\mathrm{p}} \dot{\hat{Q}}+\gamma_{p}\left(z_{\bar{p}}-\hat{\bar{p}}\right)
$$

with $z_{\pi}$ and $z_{\bar{p}}$ the respective measurements. 


\subsection{Elastodynamics-Stokes coupling}

In order to better understand the behavior of Luenberger filters in FSI, we isolated so far two physical phenomena: the added-mass effect and the dissipation coming from boundary conditions. In this section, we introduce a simplified model which includes a new physical property, namely, the dissipation in the 3D fluid.

We consider a linear FSI problem consisting of the Stokes equations, set in a fixed domain, with free outflow boundary conditions:

$$
\left\{\begin{aligned}
\rho_{\mathrm{f}} \partial_{t} \boldsymbol{u}_{\mathrm{f}}-\boldsymbol{\nabla} \cdot \boldsymbol{\sigma}_{\mathrm{f}}\left(\boldsymbol{u}_{\mathrm{f}}, p\right)=\mathbf{0}, & \text { in } \Omega_{0}^{\mathrm{f}} \\
\boldsymbol{\nabla} \cdot \boldsymbol{u}_{\mathrm{f}}=0, & \text { in } \Omega_{0}^{\mathrm{f}} \\
\boldsymbol{u}_{\mathrm{f}}=\boldsymbol{u}_{\mathrm{in}}, & \text { on } \Gamma^{\text {in }} \\
\boldsymbol{\sigma}_{\mathrm{f}}\left(\boldsymbol{u}_{\mathrm{f}}, p\right) \cdot \boldsymbol{n}_{\mathrm{f}}=0, & \text { on } \Gamma^{\text {out }}
\end{aligned}\right.
$$

coupled to the solid equations (1c) by the usual transmission conditions. This yields the following discrete dynamical system

$$
\begin{aligned}
& {\left[\begin{array}{cccccc}
K_{\mathrm{s}, \mathrm{I}} & K_{\mathrm{s}, \mathrm{I} \Sigma} & 0 & 0 & 0 & 0 \\
K_{\mathrm{s}, \mathrm{I} \Sigma}^{\top} & K_{\mathrm{s}, \Sigma} & 0 & 0 & 0 & 0 \\
0 & 0 & M_{\mathrm{s}, \mathrm{I}} & M_{\mathrm{s}, \mathrm{I} \Sigma} & 0 & 0 \\
0 & 0 & M_{\mathrm{s}, \mathrm{I} \Sigma}^{\top} & M_{\mathrm{s}, \Sigma}+M_{\mathrm{f}, \Sigma} & M_{\mathrm{f}, \mathrm{I} \Sigma}^{\top} & 0 \\
0 & 0 & 0 & M_{\mathrm{f}, \mathrm{I} \Sigma} & M_{\mathrm{f}, \mathrm{I}} & 0 \\
0 & 0 & 0 & 0 & 0 & 0
\end{array}\right]\left[\begin{array}{c}
\dot{Y}_{\mathrm{s}, \mathrm{I}} \\
\dot{Y}_{\mathrm{s}, \Sigma} \\
\dot{U}_{\mathrm{s}, \mathrm{I}} \\
\dot{U}_{\Sigma} \\
\dot{U}_{\mathrm{f}, \mathrm{I}} \\
\dot{P}
\end{array}\right] } \\
&=-\left[\begin{array}{cccccc}
-K_{\mathrm{s}, \mathrm{I}} & -K_{\mathrm{s}, \mathrm{I} \Sigma} & 0 & 0 & 0 & 0 \\
-K_{\mathrm{s}, \mathrm{I} \Sigma}^{\top} & -K_{\mathrm{s}, \Sigma} & 0 & 0 & 0 & 0 \\
K_{\mathrm{s}, \mathrm{I}} & K_{\mathrm{s}, \mathrm{I} \Sigma} & C_{\mathrm{s}, \mathrm{I}} & C_{\mathrm{s}, \mathrm{I} \Sigma} & 0 & 0 \\
K_{\mathrm{s}, \mathrm{I} \Sigma}^{\top} & K_{\mathrm{s}, \Sigma} & C_{\mathrm{s}, \mathrm{I} \Sigma}^{\top} & C_{\mathrm{s}, \Sigma}+K_{\mathrm{f}, \Sigma} & K_{\mathrm{f}, \mathrm{I} \Sigma}^{\top} & B_{\mathrm{f}, \Sigma}^{\top} \\
0 & 0 & 0 & K_{\mathrm{f}, \mathrm{I} \Sigma} & K_{\mathrm{f}, \mathrm{I}} & B_{\mathrm{f}, \mathrm{I}}^{\top} \\
0 & 0 & 0 & B_{\mathrm{f}, \Sigma} & B_{\mathrm{f}, \mathrm{I}} & -K_{p}^{\varepsilon}
\end{array}\right]\left[\begin{array}{c}
Y_{\mathrm{s}, \mathrm{I}} \\
Y_{\mathrm{s}, \Sigma} \\
U_{\mathrm{s}, \mathrm{I}} \\
U_{\Sigma} \\
U_{\mathrm{f}, \mathrm{I}} \\
P
\end{array}\right],
\end{aligned}
$$

where we distinguish, both in the fluid and in the solid, the degrees of freedom attached to the interface from those in the interior of each domain, denoting $Y_{\mathrm{s}} \stackrel{\text { def }}{=}\left[Y_{\mathrm{s}, \mathrm{I}}, Y_{\Sigma}\right]^{\top}, U_{\mathrm{s}} \stackrel{\text { def }}{=}\left[U_{\mathrm{s}, \mathrm{I}}, U_{\Sigma}\right]^{\top}$ and $U_{\mathrm{f}} \stackrel{\text { def }}{=}\left[U_{\Sigma}, U_{\mathrm{f}, \mathrm{I}}\right]^{\top}$. The fluid pressure degrees of freedom are denoted by $P$. The fluid $\left(K_{\mathrm{f}}\right.$, $M_{\mathrm{f}}$ and $\left.B_{\mathrm{f}}\right)$ and solid $\left(K_{\mathrm{s}}, C_{\mathrm{s}}\right.$ and $\left.M_{\mathrm{S}}\right)$ matrices are defined by blocks, where the subscripts $I$ and $\Sigma$ refer to the internal and interface entries, respectively. In particular, we have

$$
\begin{gathered}
\forall \boldsymbol{u}_{\mathrm{f}}, \boldsymbol{v}_{\mathrm{f}} \in \mathcal{V}_{f, h}, \quad V_{\mathrm{f}}^{\top} K_{\mathrm{f}} U_{\mathrm{f}}=\int_{\Omega_{0}^{\mathrm{f}}} 2 \mu_{\mathrm{f}} \boldsymbol{\varepsilon}\left(\boldsymbol{u}_{\mathrm{f}}\right): \varepsilon\left(\boldsymbol{v}_{\mathrm{f}}\right) d \Omega, \\
\forall \boldsymbol{u}_{\mathrm{f}}, \boldsymbol{v}_{\mathrm{f}} \in \mathcal{V}_{f, h}, \quad V_{\mathrm{f}}^{\top} M_{\mathrm{f}} U_{\mathrm{f}}=\int_{\Omega_{0}^{\mathrm{f}}} \rho_{\mathrm{f}} \boldsymbol{u}_{\mathrm{f}} \cdot \boldsymbol{v}_{\mathrm{f}} d \Omega \\
\forall \boldsymbol{v}_{\mathrm{f}} \in \mathcal{V}_{f, h}, p \in \mathcal{V}_{p, h} \quad V_{\mathrm{f}}^{\top} B_{\mathrm{f}}^{\top} P=-\int_{\Omega_{0}^{\mathrm{f}}} p \boldsymbol{\nabla} \cdot \boldsymbol{v}_{\mathrm{f}} d \Omega .
\end{gathered}
$$

The term $K_{p}$ corresponds to the pressure stabilization operator, which here is given by

$$
\forall p, q \in \mathcal{V}_{p, h} \quad Q^{\top} K_{p}^{\varepsilon} P=\varepsilon_{p} \int_{\Omega_{0}^{\mathrm{f}}} \frac{h^{2}}{\mu_{\mathrm{f}}} \nabla p \cdot \nabla q d \Omega,
$$


with $\varepsilon_{p}>0$ the stabilization parameter (see, e.g., [11]).

The construction of the observers is performed, as before for the simplified fluid models, by adding the corresponding feedback terms for the DVF and SDF in the structure equations block (i.e., rows $1-3$ of $(60))$.

Spectral numerical investigations and analysis. For the isolated Stokes problem (i.e., with homogeneous Dirichlet data on $\Sigma$ ),

$$
\left[\begin{array}{cc}
M_{\mathrm{f}, \mathrm{I}} & 0 \\
0 & 0
\end{array}\right]\left[\begin{array}{c}
\dot{U}_{\mathrm{f}, \mathrm{I}} \\
\dot{P}
\end{array}\right]=-\left[\begin{array}{cc}
K_{\mathrm{f}, \mathrm{I}} & B_{\mathrm{f}, \mathrm{I}}^{\mathrm{T}} \\
-B_{\mathrm{f}, \mathrm{I}} & K_{p}^{\varepsilon}
\end{array}\right]\left[\begin{array}{c}
U_{\mathrm{f}, \mathrm{I}} \\
P
\end{array}\right],
$$

all the poles are of course real negative. For the FSI system, some poles are complex, as in all the previous examples, and some are real negative, because of the dissipation in the fluid. We studied the behavior of the first 100 poles of smallest modulus. Note that, with the physical parameters considered in this paper, all these poles are real (the complex poles in the FSI case have a very large imaginary part, indeed).

We observe in Figure 16-left that these poles are almost the same for the isolated Stokes and the FSI system (60). To study the effect of the filters on the poles, we considered for the first two FSI poles the perturbation $\lambda_{\gamma}-\lambda_{\mid 0}$ for a gain $\gamma=200$. These results are reported in Figure 16 (right). We observe that the poles are practically insensitive to $\gamma$. In other words, the poles coming from the fluid viscosity are almost unaffected by the DVF and SDF filters.

This behavior can be better understood by examining the orders of magnitude of the various terms arising in the modal equations, as we did in the case of the Windkessel model effect in Section 3.3. We denote here a mode by $\left[\Phi_{S, d \mid 0}, \Phi_{S, v \mid 0}, \Phi_{S, f \mid 0}, \Phi_{S, p \mid 0}\right]$ - where again each of $\Phi_{S, d \mid 0}$, $\Phi_{S, v \mid 0}$ and $\Phi_{S, \mathrm{f} \mid 0}$ can be split into interface and interior parts, with the boundary condition $\left.\Phi_{S, v \mid 0}\right|_{\Sigma}=\left.\Phi_{S, \mathrm{f} \mid 0}\right|_{\Sigma}$. Considering a real eigenvector associated with a real negative eigenvalue $\lambda_{S \mid 0}^{\text {real }}$, the modal equation directly follows from (60), and the corresponding natural energy scaling reads

$$
\Phi_{S, d \mid 0}^{\text {real }}{ }^{\top} K_{\mathrm{S}} \Phi_{S, d \mid 0}^{\text {real }}+\Phi_{S, v \mid 0}^{\text {real }}{ }^{\top} M_{\mathrm{S}} \Phi_{S, v \mid 0}^{\text {real }}+\Phi_{S, \mathrm{f} \mid 0}^{\text {real }}{ }^{\top} M_{\mathrm{f}} \Phi_{S, \mathrm{f} \mid 0}^{\text {real }}=1
$$

Similarly as before, we have

$$
\frac{\Phi_{S, v \mid 0}^{\text {real }}{ }^{\top} M_{\mathrm{S}} \Phi_{S, v \mid 0}^{\text {real }}}{\Phi_{S, d \mid 0}^{\text {real }}{ }^{\top} K_{\mathrm{S}} \Phi_{S, d \mid 0}^{\mathrm{real}}} \leq\left(\frac{\left|\lambda_{S \mid 0}^{\mathrm{real}}\right|}{\omega^{\mathrm{min}}}\right)^{2} \ll 1,
$$

for the range of values of the poles considered here - recall Figure 16-left $-\omega^{\text {min }}$ denoting the fundamental natural frequency of the solid alone, still of the same order $O(100)$. In addition, here for a rather regular coupled eigenvector with a smooth transition at the fluid-solid interface, we would have the ratio $\left(\Phi_{S, v \mid 0}^{\top} M_{\mathrm{S}} \Phi_{S, v \mid 0}\right) /\left(\Phi_{S, \mathrm{f} \mid 0}^{\top} M_{\mathrm{f}} \Phi_{S, \mathrm{f} \mid 0}\right)$ comparable to the solid-to-fluid mass ratio, equal to about 0.25 in our case. Hence, the solid elastic energy drastically dominates in such coupled eigenvectors, unless the solid modal displacements altogether vanish. In fact, by inspection it can be seen that this is precisely what happens for the poles concerned here, with a penalization phenomenon which tends to enforce vanishing solid elastic and kinetic energy, and accordingly the limit modal problem corresponds to that associated with the isolated Stokes problem with homogeneous Dirichlet boundary conditions on $\Sigma$, namely (61). This also explains why the filters have very little effects on these poles of negligible solid energy.

This analysis thus provides insight in the behavior observed in Example 1 (Figures 3 and 4), where the error curves in the fluid have a similar decay rate with and without the filter in the end of the cardiac cycle. The decay rate approximately corresponds to these pure real poles that are almost not perturbed by the solid filters. A similar comment can be made for Example 2 (Figures 
7 and 8), namely at the end of the cardiac cycle, the decay of the total error is controlled by the poles of the Stokes problem and is almost unaffected by the filters. Hence, the effectiveness of the SDF in the two examples is explained by the fact that the filter operates on a system with small state error in the fluid itself - due to the choice of initial conditions - while the SDF is directly effective on the other constituents in the system.

These observations show that it would be desirable to complement solid measurements with blood flow measurements. In this respect, ultrasound (US) is still the most widespread imaging modality to inspect blood flows, but MRI acquisition sequences developed over the last two decades offer better image quality. Phase Contrast (PC) MRI can provide the flow speed in one direction over a few slices along the vessel. By acquiring the data in multiple directions, this technique provides 3D blood flow data. PC cine MRI generates "4D" blood flow data, i.e. $3 \mathrm{D}$ blood flow throughout the cardiac cycle. In some circumstances, pressure can also be acquired directly by catheters. Including these data in an observer would certainly improve the results obtained for FSI systems. This will be considered in future works. We also point out that other approaches have already been studied for assimilating flow data. In particular, in [23] a simplified variational approach was used, by which the discrepancy between model and measurements is minimized independently at each time step. Moreover, in [39, 25] Least Squares Finite Element methods were applied for stationary flows, by writing the partial differential equations as an (energy) minimization problem, and then penalizing the discrepancy between model and measurements. In [40,34], a feedback-based approach was already applied to the assimilation of ultrasound data in an aorta.

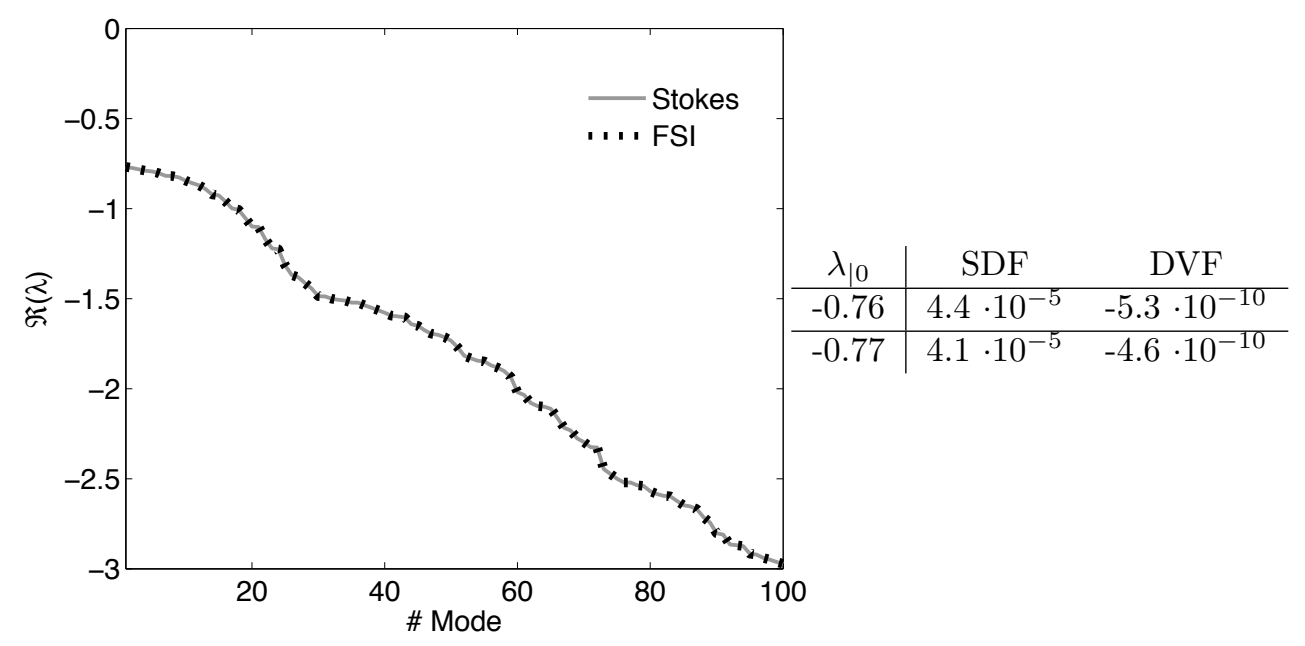

Figure 16: Real poles of the FSI system compared to the Stokes poles (left), and effect of the DVF and SDF represented by the values of $\lambda_{\gamma}-\lambda_{\mid 0}$, for $\gamma_{d}=\gamma_{v}=\gamma=200$ (right) 


\section{Discussion}

\subsection{Choice of the feedback gain in FSI problems}

In the previous section, we investigated Luenberger observers in FSI problems. The aim was not only to understand the difference of performance, but also to find a systematic method for choosing the "optimal" feedback gain, i.e. the coefficient $\gamma$ in (11) which corresponds to the fastest stabilization.

As already mentioned in Section 3.4, if we search for the eigenvalues of smallest modulus, we will not find the non-real poles since the modulus of the complex poles is much larger than the modulus of the real ones (around 100 times larger with the physiological parameters of blood flows). Since we are interested in the effect of the filters on the stability of the system, it would be natural to search the eigenvalues with the largest real part. For this purpose, we could apply for example the algorithm used in [31] based on special Cayley transforms. However, we observed that this method may fail when the imaginary part is much larger than the real part. Moreover, it may be difficult to follow the trajectories of the poles when increasing the gain.

In view of these difficulties, we suggest in practice to calibrate the feedback gain by considering the simplified FSI system with added mass-effect (41). For example, for the SDF, a first guess can be obtained with (44), assuming full observations and no viscosity in the solid. A simple computation gives $\lambda^{2}+\gamma_{d} \lambda+\omega_{a}^{2}=0$. Hence, the optimal value of $\gamma_{d}$ (i.e. which makes $\Im(\lambda)=0$ ) is given by $\gamma_{d}=2 \omega_{a}^{\min }$, where $\omega_{a}^{\min }$ is the first natural frequency of problem (45). Then, a more refined value can be obtained by considering the right observation operator and solid viscosity.

\subsection{Some inefficient alternative approaches}

In this section, we briefly comment on two other approaches that may seem natural but lead to very inefficient filters, which further justifies the analysis given in the previous section. In other words, even if the complete proof of the stabilization efficiency of the SDF for FSI is far beyond the scope of this article, the eigenvalue sensitivities allow us to eliminate some inefficient alternative approaches.

SDF by coupling with $U_{\mathrm{f}}=\dot{Y}_{\mathrm{s}}$ on $\Sigma$. We remind that the SDF modifies the usual relationship between the velocity and the displacement in the solid. For the FSI problem, we advocated to transmit the solid velocity to the fluid $\left(U_{\mathrm{f}}=U_{\mathrm{s}}\right)$. It is natural to ask what whould happen if we were to instead enforce $U_{\mathrm{f}}=\dot{Y}_{\mathrm{s}}$. This subtle difference has dramatic effects because the added-mass does not appear in the dissipative term anymore. Indeed, the quadratic eigenvalue problem becomes

Find $\left(\Phi_{d}, \lambda\right)$ such that

$$
\left(\lambda^{2}\left(M_{\mathrm{s}}+M_{A}\right)+\lambda\left(C_{\mathrm{s}}+\gamma_{d} M_{\mathrm{s}} K_{\epsilon}^{-1} H_{d}^{\top} M_{*} H_{d}\right)+K_{\mathrm{s}}+\gamma_{d} C_{\mathrm{s}} K_{\epsilon}^{-1} H_{d}^{\top} M_{*} H_{d}\right) \Phi_{d}=0 .
$$

Then, we can check the lower performance of this SDF, that will be called SDFd, in both spectral and nonlinear transient analysis shown in Figure 17 (Example 1, and equations (40) for the spectral analysis) and Figure 18 (Example 2, and equations (51) for the spectral analysis). Note that the SDFd is also inefficient to stabilize the Windkessel pole.

Force displacement feedback (FDF). Instead of using the SDF which modifies the relationship between the velocity and the displacement, it may be tempting to directly apply a collocated 

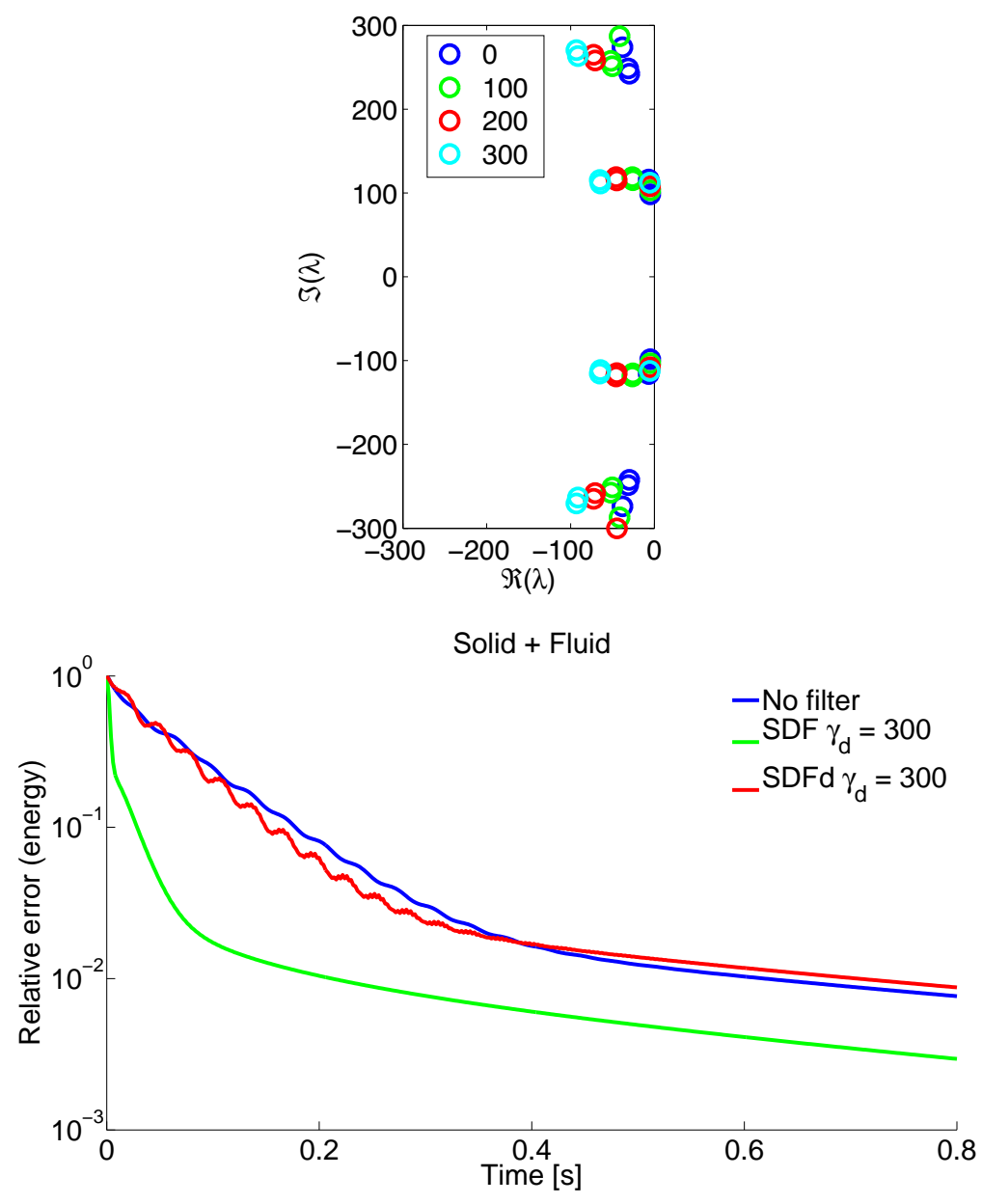

Figure 17: Linear spectral (left) and nonlinear transient (right) analysis for Example 1 with the SDFd 

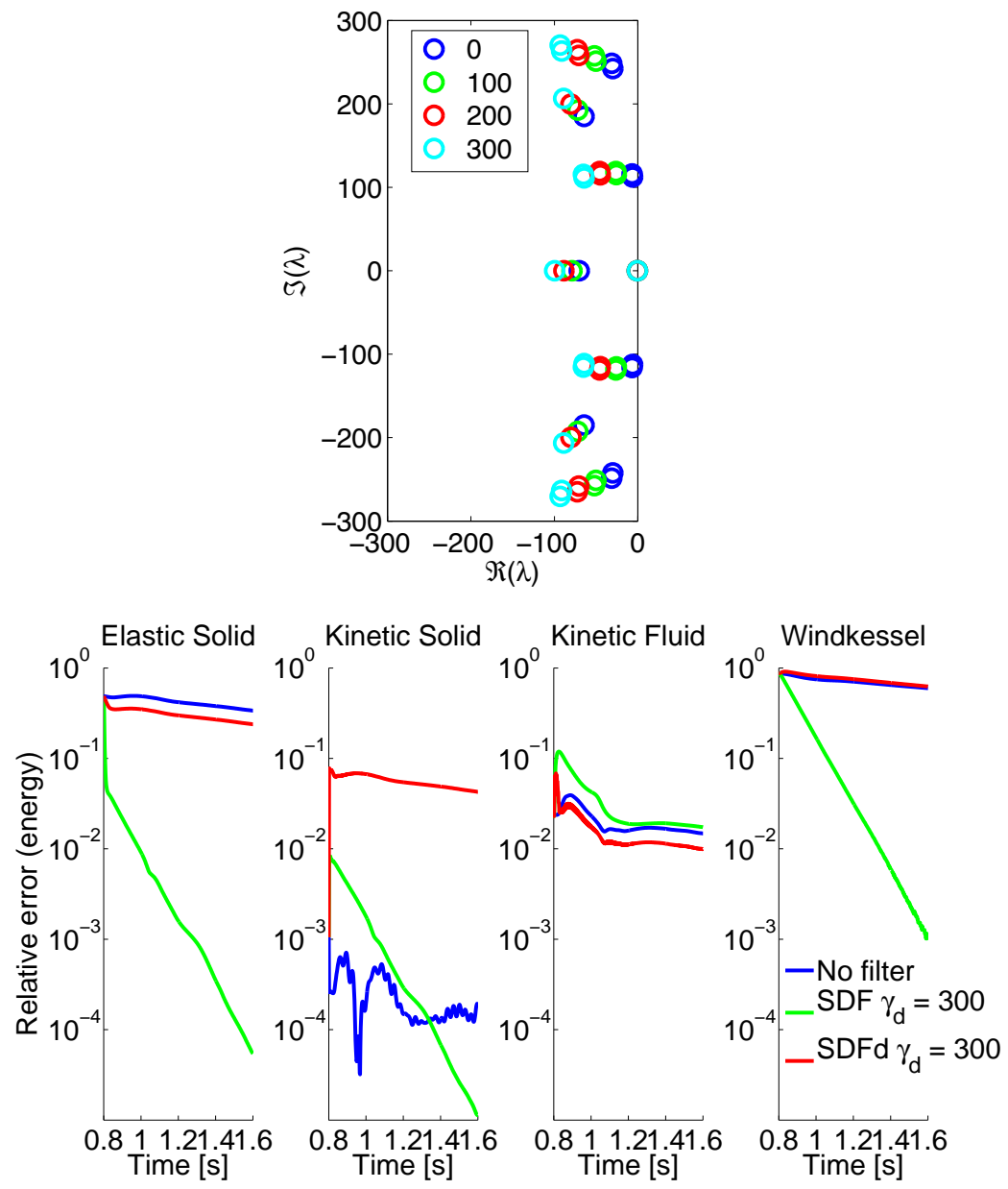

Figure 18: Linear spectral (left) and nonlinear transient (right) analysis for Example 2 with the SDFd 
displacement feedback in the momentum equation, i.e.,

$$
\left(M_{\mathrm{s}}+M_{A}\right) \ddot{\widehat{Y}}_{\mathrm{s}}+C_{\mathrm{s}} \dot{\widehat{Y}}_{\mathrm{s}}+K_{\mathrm{s}} \widehat{Y}_{\mathrm{s}}=\gamma_{d} H_{d}^{\top} M_{*}\left(Z-H_{d} \widehat{Y}_{\mathrm{s}}\right)
$$

This approach is related to the so-called image force methods, see e.g. [10]. The associated quadratic eigenvalue problem has the form

$$
\text { Find }\left(\Phi_{d}, \lambda\right) \text { such that }\left(\lambda^{2}\left(M_{\mathrm{s}}+M_{A}\right)+\lambda C_{\mathrm{s}}+K_{\mathrm{s}}+\gamma_{d} H_{d}^{\top} M_{*} H_{d}\right) \Phi_{d}=0 \text {. }
$$

This filter, which acts as an added-stiffness, is known to be efficient only for systems with very large natural dissipation. Figure 19, left, shows that the FDF behaves poorly in the linear case (51). In Figure 19, right, this weak performance is confirmed in the nonlinear transient case (Example 2), in spite of the additional dissipation due to the fluid viscosity.
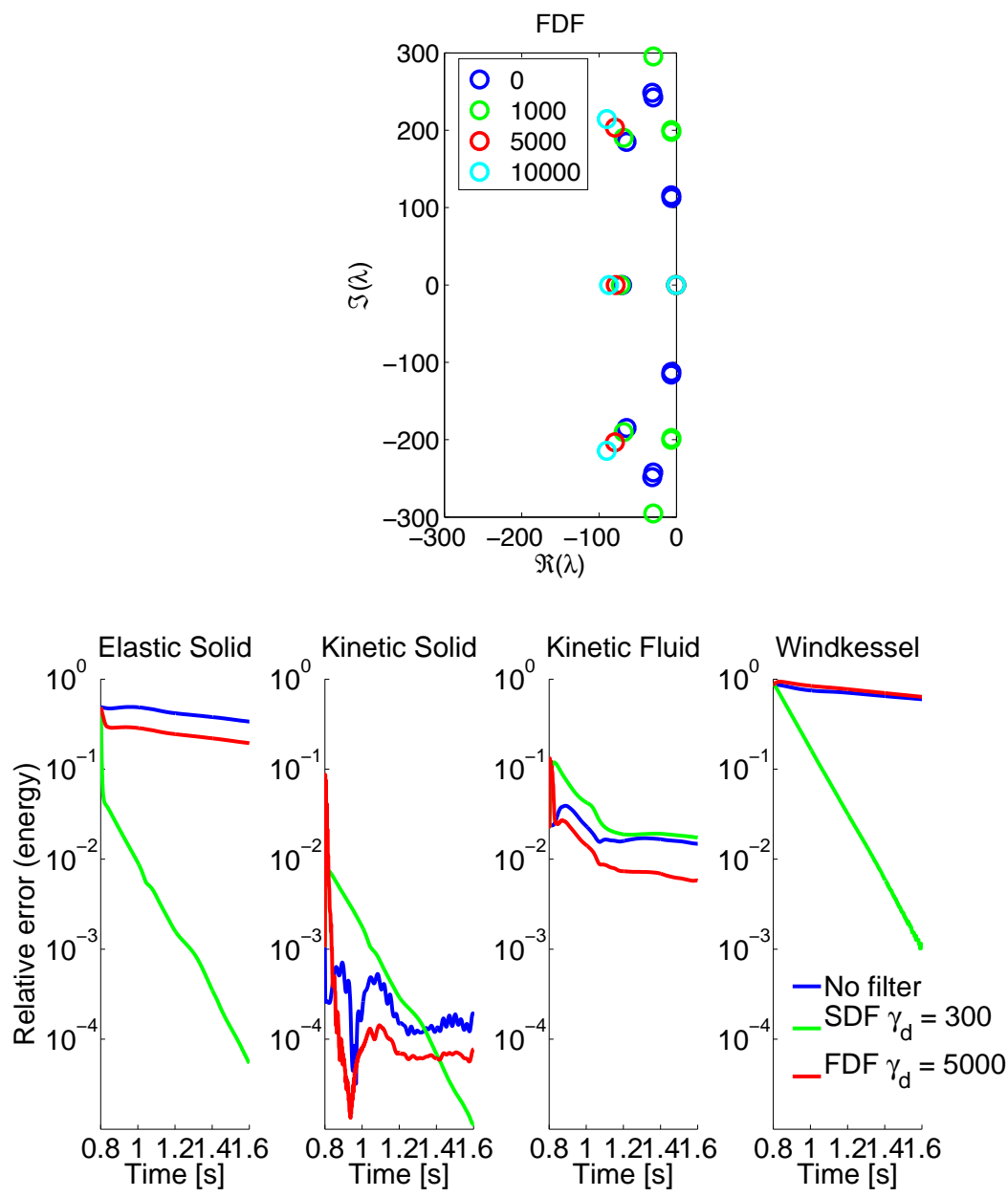

Figure 19: Effect of FDF on the FSI+Windkessel system: spectral analysis with a potential fluid (left) and nonlinear transient analysis (right) 


\section{Conclusions and perspectives}

We analyzed various sequential procedures to reduce the uncertainty in the initial condition of systems involving a viscous incompressible fluid and an elastic structure, assuming that measurements are available only for the solid velocities or displacements, as is commonly the case in practice.

We recalled two strategies, named DVF and SDF, devised for elastodynamics, and we analyzed them for FSI, with a special emphasis on hemodynamics-inspired problems. We found that the fluid can strongly impact the filters performances via three different phenomena: the added-mass effect, the coupling with a lumped-parameter model, and fluid dynamics effects per se. The SDF filter - using displacement measurements - was found to be very robust with respect to the first two, provided the coupling variable is adequately chosen to avoid perturbations due to the modified displacement-velocity relation. In the case of velocity measurements and the DVF filter, coping with the added-mass effect requires some adaptations and we proposed to include the added-mass in the scalar product used for the observation, which restores the good performance obtained in solid mechanics. When taking into account the coupling with a Windkessel model, only the SDF filter proved effective, due to the quasi-static nature of the corresponding new pole. Finally, our analysis showed that both filters applied only in the solid have almost no impact on the poles corresponding to the fluid viscosity. In order to circumvent this difficulty - which can be directly attributed to limited observability of the fluid dynamics when restricting the measurements to the fluid-solid interface - we need to rely on additional measurements in the fluid, which will be presented in future works. Nevertheless, the SDF filter is directly applicable when the initial condition is well-known in the fluid, as illustrated in the initial examples.

Therefore, our analysis has brought important insight into how Luenberger observers can be devised for state estimation in typical FSI problems arising in hemodynamics, thus providing a most effective alternative to classical variational procedures. We finally emphasize that this type of state estimation approach can be very conveniently and effectively combined with other observer techniques targeted at parameter estimation - such as that presented in [9] for the same type of FSI problems - along the lines of the overall strategy originally proposed in [51, 50].

Acknowledgement. The research leading to these results has received funding from the European Community's Seventh Framework Program (FP7/2007-2013) under grant agreement $n^{\circ}$ 224495 (euHeart project).

\section{A Properties of the discretization of the harmonic lifitng}

Exact discretized extension We introduce $L_{\mathrm{s}, *}$ the discretization of the extension operator $\mathrm{Ext}_{*}^{\mathrm{s}}$. Following [52], this discrete operator can be defined by

$$
L_{\mathrm{s}, *}: Z_{d} \mapsto \underset{H_{d} D=Z_{d}}{\operatorname{argmin}} \frac{1}{2} D_{\mathrm{s}}^{\top} K_{\mathrm{s}} D_{\mathrm{s}} .
$$

Any $D_{\mathrm{s}}$ associated with $\boldsymbol{d}_{\mathrm{s}} \in \mathcal{V}_{s, h}$ can be trivially decomposed as

$$
D_{\mathrm{s}}=D_{\mathrm{s}}^{\sharp}+L_{\mathrm{s}, *} H_{d} D_{\mathrm{s}}, \quad \text { with } D_{\mathrm{s}}^{\sharp}=D_{\mathrm{s}}-L_{\mathrm{s}, *} H_{d} D_{\mathrm{s}},
$$

so that it holds that $H_{d}\left(D_{\mathrm{s}}^{\sharp}\right)=0$. Then, by definition of the minimum in (64), we have the following identity for any data vector $Z_{d}$

$$
\left(D_{\mathrm{s}}^{\sharp}\right)^{\top} K_{\mathrm{s}} L_{\mathrm{s}, *} Z_{d}=0,
$$


which is the discrete counterpart of (15a). Hence, for any displacement vectors $D_{\mathrm{s}}$ and $Y_{\mathrm{s}}$

$$
D_{\mathrm{s}}^{\top} K_{\mathrm{s}} L_{\mathrm{s}, *} H_{d} Y_{\mathrm{s}}=D_{\mathrm{s}}^{\top} H_{d}^{\top} L_{\mathrm{s}, *}^{\top} K_{\mathrm{s}} L_{\mathrm{s}, *} H_{d} Y_{\mathrm{s}} .
$$

Penalized discretized extension For the operator $L_{\mathrm{s}, *}^{\epsilon}$ we first have, recalling (33),

$$
K_{\mathrm{s}} L_{\mathrm{s}, *}^{\epsilon} H_{d}=K_{\mathrm{s}}\left(\epsilon K_{\mathrm{s}}+H_{d}^{\top} M_{*} H_{d}\right)^{-1} H_{d}^{\top} M_{*} H_{d}=K_{\mathrm{s}}-\epsilon K_{\mathrm{s}}\left(\epsilon K_{\mathrm{s}}+H_{d}^{\top} M_{*} H_{d}\right)^{-1} K_{\mathrm{s}},
$$

which already shows that $K_{\mathrm{s}} L_{\mathrm{s}, *}^{\epsilon} H_{d}$ is a symmetric operator. Hence, for any vector $D_{\mathrm{s}}$,

$$
K_{\mathrm{s}}\left(D_{\mathrm{s}}-L_{\mathrm{s}, *}^{\epsilon} H_{d} D_{\mathrm{s}}\right)=\epsilon K_{\mathrm{s}}\left(\epsilon K_{\mathrm{s}}+H_{d}^{\top} M_{*} H_{d}\right)^{-1} K_{\mathrm{s}} D_{\mathrm{s}}
$$

giving, for any $D_{\mathrm{s}}$ and $Y_{\mathrm{s}}$,

$$
\begin{aligned}
\left(D_{\mathrm{s}}-L_{\mathrm{s}, *}^{\epsilon} H_{d} D_{\mathrm{s}}\right)^{\top} K_{\mathrm{s}} L_{\mathrm{s}, *}^{\epsilon} H_{d} Y_{\mathrm{s}}= & \epsilon D_{\mathrm{s}}^{\top} K_{\mathrm{s}}\left(\epsilon K_{\mathrm{s}}+H_{d}^{\top} M_{*} H_{d}\right)^{-1} K_{\mathrm{s}} Y_{\mathrm{s}} \\
& -\epsilon^{2} D_{\mathrm{s}}^{\top} K_{\mathrm{s}}\left(\epsilon K_{\mathrm{s}}+H_{d}^{\top} M_{*} H_{d}\right)^{-1} K_{\mathrm{s}}\left(\epsilon K_{\mathrm{s}}+H_{d}^{\top} M_{*} H_{d}\right)^{-1} K_{\mathrm{s}} Y_{\mathrm{s}} \\
= & \epsilon D_{\mathrm{s}}^{\top} K_{\mathrm{s}}\left(\epsilon K_{\mathrm{s}}+H_{d}^{\top} M_{*} H_{d}\right)^{-1} H_{d}^{\top} M_{*} H_{d}\left(\epsilon K_{\mathrm{s}}+H_{d}^{\top} M_{*} H_{d}\right)^{-1} K_{\mathrm{s}} Y_{\mathrm{s}},
\end{aligned}
$$

which represents the penalized version of the identity (65). Therefore, defining $\Gamma_{\mathrm{s}, *}^{\epsilon}=\left(\epsilon K_{\mathrm{s}}+\right.$ $\left.H_{d}^{\top} M_{*} H_{d}\right)^{-1} K_{\mathrm{s}}$ we obtain

$$
D_{\mathrm{s}}^{\top} K_{\mathrm{s}} L_{\mathrm{s}, *}^{\epsilon} H_{d} Y_{\mathrm{s}}=D_{\mathrm{s}}^{\top} H_{d}^{\top}\left(L_{\mathrm{s}, *}^{\epsilon}\right)^{\top} K_{\mathrm{s}} L_{\mathrm{s}, *}^{\epsilon} H_{d} Y_{\mathrm{s}}+\epsilon D_{\mathrm{s}}^{\top}\left(\Gamma_{\mathrm{s}, *}^{\epsilon}\right)^{\top} H_{d}^{\top} M_{*} H_{d} \Gamma_{\mathrm{s}, *}^{\epsilon} Y_{\mathrm{s}},
$$

which proves the positivity of $K_{\mathrm{s}} L_{\mathrm{s}, *}^{\epsilon} H_{d}$. Furthermore, we note that using of $L_{\mathrm{s}, *}^{\epsilon}$ instead of the exact harmonic lifting $L_{\mathrm{s}, *}$ introduces a positive operator of order $\epsilon$.

\section{B Spectral sensitivity analysis of the elastodynamics-Windkessel coupling model}

The DVF case. The solid-pressure-Windkessel modal system with the DVF reads

$$
\left\{\begin{array}{l}
K_{\mathrm{s}} \Phi_{w, v}=\lambda K_{\mathrm{s}} \Phi_{w, d} \\
-K_{\mathrm{s}} \Phi_{w, d}-\left(\gamma_{v}\left(H_{v}^{\top} M_{*} H_{v}\right)+R_{\mathrm{p}} S \cdot S^{\top}\right) \Phi_{w, v}+S \Phi_{w, \pi}=\lambda\left(M_{\mathrm{s}}+M_{A}\right) \Phi_{w, v} \\
-S^{\top} \Phi_{w, v}-\frac{1}{R_{\mathrm{d}}} \Phi_{w, \pi}=\lambda C \Phi_{w, \pi}
\end{array}\right.
$$

assuming $\eta_{\mathrm{s}}=0$ for the sake of simplicity. Rewriting it only in terms of $\Phi_{w, d}$ leads to the following eigenvalue problem

$$
\lambda^{2}\left(M_{\mathrm{s}}+M_{A}\right) \Phi_{w, d}+\gamma_{v} \lambda H_{v}^{\top} M_{*} H_{v} \Phi_{w, d}+K_{\mathrm{s}} \Phi_{w, d}+\lambda\left(\frac{1}{1+\lambda \tau}+\frac{R_{\mathrm{p}}}{R_{\mathrm{d}}}\right) R_{\mathrm{d}} S \cdot S^{\top} \Phi_{w, d}=0,
$$

with $\tau=R_{\mathrm{d}} C$. Note that this reduces to the previous elastodynamics eigenvalue problem (28) when $R_{\mathrm{p}}, R_{\mathrm{d}}$ and $C$ equal zero. As done previously, we can compute the sensitivity of $\lambda$ with respect to $\gamma_{v}$ by differentiating (67) at $\gamma_{v}=0$. We get

$$
\begin{aligned}
& \left.2 \lambda_{w \mid 0} \frac{\partial \lambda}{\partial \gamma_{v}}\right|_{\gamma_{v}=0}\left(M_{\mathrm{s}}+M_{A}\right) \Phi_{w, d \mid 0}+ \\
& \lambda_{w \mid 0} H_{v}^{\top} M_{*} H_{v} \Phi_{w, d \mid 0}+\left.\frac{\partial}{\partial \lambda}\left(\frac{\lambda}{1+\lambda \tau}+\lambda \frac{R_{\mathrm{p}}}{R_{\mathrm{d}}}\right) \frac{\partial \lambda}{\partial \gamma_{v}}\right|_{\gamma_{v}=0} R_{\mathrm{d}} S \cdot S^{\top} \Phi_{w, d \mid 0} \\
& +\left.\left(\lambda_{w \mid 0}^{2}\left(M_{\mathrm{s}}+M_{A}\right) \Phi_{w, d \mid 0}+K_{\mathrm{s}} \Phi_{w, d \mid 0}+\lambda_{w \mid 0}\left(\frac{1}{1+\lambda_{w \mid 0} \tau}+\frac{R_{\mathrm{p}}}{R_{\mathrm{d}}}\right) R_{\mathrm{d}} S \cdot S^{\top} \Phi_{w, d \mid 0}\right) \frac{\partial \Phi_{w, d}}{\partial \gamma_{v}}\right|_{\gamma_{v}=0}=0,
\end{aligned}
$$


with $\left(\Phi_{w, d \mid 0}, \lambda_{w \mid 0}\right)$ solution of

$$
\lambda_{w \mid 0}^{2}\left(M_{\mathrm{s}}+M_{A}\right) \Phi_{w, d \mid 0}+K_{\mathrm{s}} \Phi_{w, d \mid 0}+\lambda_{w \mid 0}\left(\frac{1}{1+\lambda_{w \mid 0} \tau}+\frac{R_{\mathrm{p}}}{R_{\mathrm{d}}}\right) R_{\mathrm{d}} S \cdot S^{\top} \Phi_{w, d \mid 0}=0 .
$$

We now consider a specific real eigenvector $\Phi_{w, d \mid 0}^{\text {real }}$ associated with a real negative eigenvalue

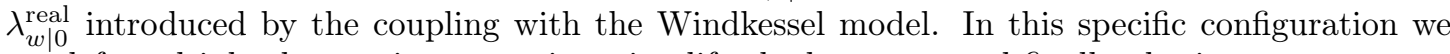
can left multiply the previous equation, simplify the last part, and finally obtain

$$
\begin{aligned}
& \left.\frac{\partial \lambda^{\text {real }}}{\partial \gamma_{v}}\right|_{\gamma_{v}=0}= \\
& \quad-\frac{\left(\Phi_{w, d \mid 0}^{\text {real }}\right)^{\top} H_{v}^{\top} M_{*} H_{v} \Phi_{w, d \mid 0}^{\text {real }}}{2\left(\Phi_{w, d \mid 0}^{\text {real }}\right)^{\top}\left(M_{\mathrm{S}}+M_{A}\right) \Phi_{w, d \mid 0}^{\text {real }}+\left(\frac{1}{\left(1+\lambda_{w \mid 0}^{\text {real }} \tau\right)^{2}}+\frac{R_{\mathrm{p}}}{R_{\mathrm{d}}}\right) \frac{1}{\lambda_{w \mid 0}^{\text {real }}} R_{\mathrm{d}}\left(\Phi_{w, d \mid 0}^{\text {real }}\right)^{\top} S \cdot S \top \Phi_{w, d \mid 0}^{\text {real }}} .
\end{aligned}
$$

Recalling our discussion in Section 3.3, we have by (54)-(55)

$$
\begin{aligned}
\left(\Phi_{w, d \mid 0}^{\text {real }}\right)^{\top}\left(M_{\mathrm{S}}+M_{A}\right) \Phi_{w, d \mid 0}^{\text {real }} & =\frac{1}{\left(\lambda_{w \mid 0}^{\text {real }}\right)^{2}}\left(\Phi_{w, v \mid 0}^{\text {real }}\right)^{\top}\left(M_{\mathrm{s}}+M_{A}\right) \Phi_{w, v \mid 0}^{\text {real }} \\
& \ll \frac{1}{\left(\lambda_{w \mid 0}^{\text {real }}\right)^{2}}\left(\Phi_{w, d \mid 0}^{\text {real }}\right)^{\top} K_{\mathrm{s}} \Phi_{w, d \mid 0}^{\text {real }} \approx \frac{1}{\left(\lambda_{w \mid 0}^{\text {real }}\right)^{2}} S^{\top}\left(K_{\mathrm{S}}\right)^{-1} S\left(\Phi_{w, \pi}\right)^{2} .
\end{aligned}
$$

Hence, with (56) we infer

$$
\left(\Phi_{w, d \mid 0}^{\text {real }}\right)^{\top}\left(M_{\mathrm{s}}+M_{A}\right) \Phi_{w, d \mid 0}^{\text {real }} \ll R_{\mathrm{d}}^{2}\left(C+S^{\top}\left(K_{\mathrm{s}}\right)^{-1} S\right)^{2}\left(S^{\top}\left(K_{\mathrm{s}}\right)^{-1} S\right)\left(\Phi_{w, \pi}\right)^{2},
$$

whereas for the second part in the denominator of (69) we have $1+\lambda_{w \mid 0}^{\text {real }} \tau=O(1), R_{\mathrm{p}} / R_{\mathrm{d}} \ll 1$, and

$$
\frac{1}{\lambda_{w \mid 0}^{\text {real }}} R_{\mathrm{d}}\left(\Phi_{w, d \mid 0}^{\mathrm{real}}\right)^{\top} S \cdot S^{\boldsymbol{\top}} \Phi_{w, d \mid 0}^{\mathrm{real}} \approx-R_{\mathrm{d}}^{2}\left(C+S^{\top}\left(K_{\mathrm{s}}\right)^{-1} S\right)\left(S^{\top}\left(K_{\mathrm{s}}\right)^{-1} S\right)^{2}\left(\Phi_{w, \pi}\right)^{2} .
$$

Therefore, with $C$ and $S^{\top}\left(K_{\mathrm{s}}\right)^{-1} S$ - namely, the capacitances of the Windkessel and of the static solid behavior, respectively - of the same order, we have that the first term in the denominator of (69), which would be the only one in the sensitivity without the Windkessel coupling (recall Section 3.2.1), is drastically dominated (in absolute value) by the new term, which is negative. This fully explains what was observed in Figure 15-top, namely, a very small sensitivity with a destabilization effect.

The SDF case. The eigenvalue problem for the SDF case reads

$$
\left\{\begin{array}{l}
K_{\mathrm{s}} \Phi_{w, v}=\lambda K_{\mathrm{s}} \Phi_{w, d}+\gamma_{d} K_{\mathrm{s}} L_{\mathrm{s}, *}^{\epsilon} H_{d} \Phi_{w, d} \\
-K_{\mathrm{s}} \Phi_{w, d}-R_{p} S \cdot S^{\top} \Phi_{w, v}+S \Phi_{w, \pi}=\lambda\left(M_{\mathrm{s}}+M_{A}\right) \Phi_{w, v} \\
-S^{\top} \Phi_{w, v}-\frac{1}{R_{d}} \Phi_{w, \pi}=\lambda C \Phi_{w, \pi}
\end{array}\right.
$$

which, rewritten only in terms of $\Phi_{w, d}$, leads to the following cubic eigenvalue problem

$$
\begin{aligned}
\lambda^{2}\left(M_{\mathrm{s}}+M_{A}\right) \Phi_{w, d}+\gamma_{d}\left(\left(\frac{1}{1+\lambda \tau}+\right.\right. & \left.\left.\frac{R_{\mathrm{p}}}{R_{\mathrm{d}}}\right) R_{\mathrm{d}} S \cdot S^{\top}+\lambda\left(M_{\mathrm{s}}+M_{A}\right)\right) L_{\mathrm{s}, *}^{\epsilon} H_{d} \Phi_{w, d} \\
& +K_{\mathrm{s}} \Phi_{w, d}+\lambda\left(\frac{1}{1+\lambda \tau}+\frac{R_{\mathrm{p}}}{R_{\mathrm{d}}}\right) R_{\mathrm{d}} S \cdot S^{\top} \Phi_{w, d}=0
\end{aligned}
$$


Hence, after analogous computations, the sensitivity of the real poles with respect to the filter gain is given by

$$
\left.\frac{\partial \lambda^{\text {real }}}{\partial \gamma_{d}}\right|_{\gamma_{v}=0}=-\frac{\left(\Phi_{w, d \mid 0}^{\text {real }}\right)^{\top}\left(\left(\frac{1}{1+\lambda_{w \mid 0}^{\text {real }}}+\frac{R_{\mathrm{p}}}{R_{\mathrm{d}}}\right) \frac{1}{\lambda_{w \mid 0}^{\text {real }}} R_{\mathrm{d}} S \cdot S^{\top}+\left(M_{\mathrm{s}}+M_{A}\right)\right) L_{\mathrm{s}, *}^{\epsilon} H_{d} \Phi_{w, d \mid 0}^{\text {real }}}{2\left(\Phi_{w, d \mid 0}^{\text {real }}\right)^{\top}\left(M_{\mathrm{s}}+M_{A}\right) \Phi_{w, d \mid 0}^{\text {real }}+\left(\frac{1}{\left(1+\lambda_{w \mid 0}^{\text {real }} \tau\right)^{2}}+\frac{R_{\mathrm{p}}}{R_{\mathrm{d}}}\right) \frac{1}{\lambda_{w \mid 0}^{\text {real }}} R_{\mathrm{d}}\left(\Phi_{w, d \mid 0}^{\text {real }}\right)^{\top} S \cdot S \boldsymbol{\top} \Phi_{w, d \mid 0}^{\text {real }}} .
$$

Using (68), we can simplify the numerator to retrieve our symmetric operator $H_{d}^{\top}\left(L_{\mathrm{s}, *}^{\epsilon}\right)^{\top} K_{\mathrm{s}} L_{\mathrm{s}, *}^{\epsilon} H_{d}$, finally leading to

$$
\begin{aligned}
& \left.\frac{\partial \lambda^{\text {real }}}{\partial \gamma_{d}}\right|_{\gamma_{v}=0}= \\
& \frac{1}{\left(\lambda_{w \mid 0}^{\text {real }}\right)^{2}} \frac{\left(\Phi_{w, d \mid 0}^{\text {real }}\right)^{\top} H_{d}^{\top}\left(L_{\mathrm{s}, *}^{\epsilon}\right)^{\top} K_{\mathrm{s}} L_{\mathrm{s}, *}^{\epsilon} H_{d} \Phi_{w, d \mid 0}^{\text {real }}}{2\left(\Phi_{w, d \mid 0}^{\mathrm{real}}\right)^{\top}\left(M_{\mathrm{s}}+M_{A}\right) \Phi_{w, d \mid 0}^{\mathrm{real}}+\left(\frac{1}{\left(1+\lambda_{w \mid 0}^{\text {real }}\right)^{2}}+\frac{R_{\mathrm{p}}}{R_{\mathrm{d}}}\right) \frac{1}{\lambda_{w \mid 0}^{\text {real }}} R_{\mathrm{d}}\left(\Phi_{w, d \mid 0}^{\mathrm{real}}\right)^{\top} S \cdot S \top \Phi_{w, d \mid 0}^{\mathrm{real}}} .
\end{aligned}
$$

The same considerations as for the DVF imply that the second term drastically dominates in the denominator, which, combining the first quotient $1 /\left(\lambda_{w \mid 0}^{\text {real }}\right)^{2}$ is of the order of

$$
\begin{aligned}
\lambda_{w \mid 0}^{\text {real }} R_{\mathrm{d}}\left(\Phi_{w, d \mid 0}^{\text {real }}\right)^{\top} S \cdot S^{\top} \Phi_{w, d \mid 0}^{\text {real }} & \approx-\frac{\left(S^{\top}\left(K_{\mathrm{s}}\right)^{-1} S\right)^{2}}{C+S^{\top}\left(K_{\mathrm{s}}\right)^{-1} S}\left(\Phi_{w, \pi}\right)^{2} \\
& \approx-\frac{S^{\top}\left(K_{\mathrm{s}}\right)^{-1} S}{C+S^{\top}\left(K_{\mathrm{s}}\right)^{-1} S}\left(\Phi_{w, d \mid 0}^{\text {real }}\right)^{\top} K_{\mathrm{s}} \Phi_{w, d \mid 0}^{\text {real }}
\end{aligned}
$$

Since, as already discussed, the quotient here is $O(1)$, comparing with the sensitivity equation obtained in Section 3.2.1 we understand how the SDF preserves a similar overall stabilization effectiveness as without the Windkessel coupling, as seen in Figure 15-bottom.

\section{References}

[1] S. Badia, F. Nobile, and C. Vergara. Fluid-structure partitioned procedures based on Robin transmission conditions. J. Comp. Phys., 227:7027-7051, 2008.

[2] S. Balocco, O. Camara, and A. Frangi. Towards regional elastography of intracranial aneurysms. Medical Image Computing and Computer-Assisted Intervention-MICCAI 2008, pages 131-138, 2008.

[3] H.T. Banks and K. Kunisch. Estimation techniques for distributed parameter systems, volume 1 of Systems $\&$ Control: Foundations $\&$ Applications. Birkhäuser Boston Inc., Boston, MA, 1989.

[4] C. Bardos, G. Lebeau, and J. Rauch. Sharp sufficient conditions for the observation, control, and stabilization of waves from the boundary. SIAM J. Control Optim., 30(5):1024-1065, 1992.

[5] Y. Bazilevs, J.R. Gohean, T.J.R. Hughes, R.D. Moser, and Y. Zhang. Patient-specific isogeometric fluid-structure interaction analysis of thoracic aortic blood flow due to implantation of the Jarvik-2000 left ventricular assist device. Computer Methods in Applied Mechanics and Engineering, 198(45-46):3534-3550, 2009. 
[6] R.E. Bellman. Dynamic Programming. Princeton University Press, 1957.

[7] A. Bensoussan. Filtrage optimal des systèmes linéaires. Dunod, 1971.

[8] C. Bertoglio, M.A. Fernández, J-F. Gerbeau, and P. Moireau. Filtering-based data assimilation in fluid-structure interaction: towards personalization of vascular models. In P. Nithiarasu and R. Löhner, editors, 1st International Conference on Mathematical and Computational Biomedical Engineering - CMBE, 2009.

[9] C. Bertoglio, P. Moireau, and J-F. Gerbeau. Sequential parameter estimation for fluidstructure problems: Application to hemodynamics. Int. J. Numer. Meth. Biomed. Engrg., 28:434-455, 2012.

[10] F. Billet, M. Sermesant, H. Delingette, and N. Ayache. Cardiac motion recovery by coupling an electromechanical model and cine-MRI data: First steps. In K. Miller and P.M.F. Nielsen, editors, Proc. of the Workshop on Computational Biomechanics for Medicine III. (Workshop MICCAI-2008), September 2008.

[11] F. Brezzi and J. Pitkäranta. On the stabilization of finite element approximations of the Stokes equations. In Efficient solutions of elliptic systems (Kiel, 1984), volume 10 of Notes Numer. Fluid Mech., pages 11-19. Vieweg, 1984.

[12] A.N. Brooks and T.J.R. Hughes. Streamline upwind/Petrov-Galerkin formulations for convection dominated flows with particular emphasis on the incompressible Navier-Stokes equations. Comp. Meth. App. Mech. Eng., 32:199-259, 1982.

[13] N. Burq and G. Lebeau. Mesures de défaut de compacité, application au système de Lamé. Ann. Sci. Ecole Norm. Sup. (4), 34(6):817-870, 2001.

[14] P. Causin, J-F. Gerbeau, and F. Nobile. Added-mass effect in the design of partitioned algorithms for fluid-structure problems. Comput. Methods Appl. Mech. Engrg., 194(4244):4506-4527, 2005.

[15] R. Chabiniok, P. Moireau, P.-F. Lesault, A. Rahmouni, J-F. Deux, and D. Chapelle. Estimation of tissue contractility from cardiac cine-MRI using a biomechanical heart model. Biomechanics and Modeling in Mechanobiology, pages 1-22, 2011. 10.1007/s10237-011-03378 .

[16] D. Chapelle, N. Cîndea, M. de Buhan, and P. Moireau. Exponential convergence of an observer based on partial field measurements for the wave equation. Research Report 7728, INRIA, 2011. http://hal.inria.fr/inria-00619504/fr.

[17] D. Chapelle, N. Cîndea, and P. Moireau. Improving convergence in numerical analysis using observers - the wave-like equation case. M3AS, 2012. In press, doi:10.1142/S0218202512500406.

[18] D. Chapelle, P. Moireau, and P. Le Tallec. Robust filtering for joint state-parameter estimation in distributed mechanical systems. $D C D S-A, 23(1 / 2): 65-84,2009$.

[19] J.M. Coron. Control and nonlinearity. Mathematical surveys and monographs. American Mathematical Society, 2007.

[20] P. Crosetto, S. Deparis, G. Fourestey, and A. Quarteroni. Parallel algorithms for fluidstructure interaction problems in haemodynamics. SIAM J. Sci. Comput., 33(4):1598-1622, 2011.

$\mathrm{RR} \mathrm{n}^{\circ} 8177$ 
[21] R.F. Curtain and H.J. Zwart. An introduction to infinite-dimensional linear systems theory. Texts in applied mathematics. Springer-Verlag, 1995.

[22] M. D'Elia, L. Mirabella, T. Passerini, M. Perego, M. Piccinelli, C. Vergara, and A. Veneziani. Applications of variational data assimilation in computational hemodynamics. Technical Report TR-2011-002, Emory University, 2011.

[23] M. D'Elia, M. Perego, and A. Veneziani. A variational data assimilation procedure for the incompressible navier-stokes equations in hemodynamics. Journal of Scientific Computing, pages 1-20, 2011.

[24] W.G. Dettmer and D. Perić. A fully implicit computational strategy for strongly coupled fluid-solid interaction. Arch. Comput. Methods Eng., 14(3):205-247, 2007.

[25] R.P. Dwight. Bayesian inference for data assimilation using least-squares finite element methods. In IOP Conference Series: Materials Science and Engineering, volume 10, page 012224. IOP Publishing, 2010.

[26] M. Esmaily Moghadam, Y. Bazilevs, T.-Y. Hsia, I. Vignon-Clementel, A. Marsden, and Modeling of Congenital Hearts Alliance. A comparison of outlet boundary treatments for prevention of backflow divergence with relevance to blood flow simulations. Computational Mechanics, 48:277-291, 2011. 10.1007/s00466-011-0599-0.

[27] M.A. Fernández. Coupling schemes for incompressible fluid-structure interaction: implicit, semi-implicit and explicit. SëMA J., 55:59-108, 2011.

[28] M.A. Fernández and J-F. Gerbeau. Algorithms for fluid-structure interaction problems. In L. Formaggia, A. Quarteroni, and A. Veneziani, editors, Cardiovascular Mathematics. Modeling and simulation of the circulatory system, chapter 9, pages 307-346. Springer Verlag, 2009.

[29] M.A. Fernández, J-F. Gerbeau, and C. Grandmont. A projection algorithm for fluidstructure interaction problems with strong added-mass effect. C. R. Math. Acad. Sci. Paris, $342(4): 279-284,2006$.

[30] M.A. Fernández, J-F. Gerbeau, and C. Grandmont. A projection semi-implicit scheme for the coupling of an elastic structure with an incompressible fluid. Int. J. Num. Meth. Engrg., 69(4):794-821, 2007.

[31] M.A. Fernández and P. Le Tallec. Linear fluid-structure stability analysis with transpiration. part ii: numerical analysis and applications. Comput. Methods Appl. Mech. Engrg., 192(43):4837-4873, 2003.

[32] W.H. Fleming. Deterministic nonlinear filtering. Annali della Scuola Normale Superiore di Pisa - Classe di Scienze, 25:435-454, 1997.

[33] L. Formaggia, A. Moura, and F. Nobile. On the stability of the coupling of 3D and 1D fluidstructure interaction models for blood flow simulations. ESAIM-Mathematical Modelling and Numerical Analysis, 41(4):743-770, 2007.

[34] K. Funamoto, T. Hayase, Y. Saijo, and T. Yambe. Numerical experiment for ultrasonicmeasurement-integrated simulation of three-dimensional unsteady blood flow. Annals of biomedical engineering, 36(8):1383-1397, 2008. 
[35] M.W. Gee, U. Küttler, and W. Wall. Truly monolithic algebraic multigrid for fluid-structure interaction. Int. J. Numer. Meth. Engng., 85(8):987-1016, 2011.

[36] V. Gravemeier, A. Comerford, L. Yoshihara, M. Ismail, and W.A. Wall. A novel formulation for neumann inflow boundary conditions in biomechanics. International Journal for Numerical Methods in Biomedical Engineering, 2012.

[37] M. Grobbelaar-Van Dalsen. A new approach to the stabilization of a fluid-structure interaction model. Appl. Anal., 88(7):1053-1065, 2009.

[38] J.B. Grotberg and O.E. Jensen. Biofluid mechanics in flexible tubes. Annual Review of Fluid Mechanics, 36(1):121-147, 2004.

[39] J.J. Heys, T.A. Manteuffel, S.F. McCormick, M. Milano, J. Westerdale, and M. Belohlavek. Weighted least-squares finite elements based on particle imaging velocimetry data. Journal of Computational Physics, 229(1):107-118, 2010.

[40] K. Imagawa and T. Hayase. Eigenvalue analysis for error dynamics of measurement integrated simulation to reproduce real flows. Bulletin of the American Physical Society, 53, 2008 .

[41] S. Julier, J. Uhlmann, and H.F. Durrant-Whyte. A new approach for filtering nonlinear systems. In American Control Conference, pages 1628-1632, 1995.

[42] R. Kalman and R. Bucy. New results in linear filtering and prediction theory. Trans. ASME J. Basic. Eng, 83:95-108, 1961.

[43] H.J. Kim, C.A. Figueroa, T.J.R. Hughes, K.E. Jansen, and C.A. Taylor. Augmented lagrangian method for constraining the shape of velocity profiles at outlet boundaries for three-dimensional finite element simulations of blood flow. Computer Methods in Applied Mechanics and Engineering, 198(45):3551-3566, 2009.

[44] P.-Y. Lagrée. An inverse technique to deduce the elasticity of a large artery. Eur. Phys. J. $A P, 9: 153-163,1999$.

[45] I. Lasiecka and R. Triggiani. $L_{2}(\Sigma)$-regularity of the boundary to boundary operator $B^{*} L$ for hyperbolic and Petrowski PDEs. Abstr. Appl. Anal., 19:1061-1139, 2003.

[46] D.G. Luenberger. An introduction to observers. IEEE Transactions on Automatic Control, 16:596-602, 1971.

[47] Y. Maday. Analysis of coupled models for fluid-structure interaction of internal flows. In L. Formaggia, A. Quarteroni, and A. Veneziani, editors, Cardiovascular Mathematics. Modeling and simulation of the circulatory system, chapter 8, pages 279-306. Springer Verlag, 2009 .

[48] F. Magoulès, F.X. Roux, and L. Series. Algebraic approximation of dirichlet-to-neumann maps for the equations of linear elasticity. Computer Methods in Applied Mechanics and Engineering, 195(29):3742-3759, 2006.

[49] P. Moireau, C. Bertoglio, N. Xiao, C.A. Figueroa, D. Chapelle, C.A. Taylor, and J-F. Gerbeau. Sequential identification of boundary support parameters in a fluid-structure vascular using patient image data. Submitted to BMMB, 2012. 
[50] P. Moireau and D. Chapelle. Reduced-order Unscented Kalman Filtering with application to parameter identification in large-dimensional systems. COCV, 17:380-405, 2011. doi:10.1051/cocv/2010006.

[51] P. Moireau, D. Chapelle, and P. Le Tallec. Joint state and parameter estimation for distributed mechanical systems. Computer Methods in Applied Mechanics and Engineering, 197:659-677, 2008.

[52] P. Moireau, D. Chapelle, and P. Le Tallec. Filtering for distributed mechanical systems using position measurements: Perspective in medical imaging. Inverse Problems, 25(3):035010035035, March 2009.

[53] P. Moireau, N. Xiao, M. Astorino, C. A. Figueroa, D. Chapelle, C. A. Taylor, and J-F. Gerbeau. External tissue support and fluid-structure simulation in blood flows. Biomechanics and Modeling in Mechanobiology, 11(1-2):1-18, 2012.

[54] M.P. Païdoussis, S.J. Price, and E. de Langre. Fluid-Structure Interactions: Cross-flowinduced Instabilities. Cambridge University Press, 2010.

[55] J. Peiró and A. Veneziani. Reduced models of the cardiovascular system. In L. Formaggia, A. Quarteroni, and A. Veneziani, editors, Cardiovascular Mathematics. Modeling and simulation of the circulatory system, chapter 10, pages 347-394. Springer Verlag, 2009.

[56] M. Perego, A. Veneziani, and C. Vergara. A variational approach for estimating the compliance of the cardiovascular tissue: An inverse fluid-structure interaction problem. SIAM J. Sc. Comp., 33(3):1181-1211, 2011.

[57] D.T. Pham, J. Verron, and M.C. Roubeaud. A singular evolutive extended Kalman filter for data assimilation in oceanography. Journal of Marine systems, 16(3-4):323-340, 1998.

[58] A. Preumont. Vibration Control of Active Structures, An Introduction. Kluwer Academic Publishers, 2nd edition, February 2002.

[59] J-P. Raymond. Feedback stabilization of a fluid-structure model. SIAM J. Control Optim., 48(8):5398-5443, 2010.

[60] D. Simon. Optimal state estimation: Kalman, H infinity, and nonlinear approaches. WileyInterscience, 2006.

[61] J. Stalhand. Determination of human arterial wall parameters from clinical data. Biomech Model Mechanobiol, 8:141-148, 2009.

[62] X. Zhang and E. Zuazua. Long-time behavior of a coupled heat-wave system arising in fluid-structure interaction. Arch. Ration. Mech. Anal., 184(1):49-120, 2007. 


\section{Contents}

1 Introduction $\quad 3$

2 Observer for the fluid-structure interaction problem $\quad 4$

2.1 Fluid-structure interaction equations . . . . . . . . . . . . . . . . . . . 4

2.2 Observer based on solid measurements . . . . . . . . . . . . . . . . . . . . 7

2.2.1 Discrepancy measure minimization . . . . . . . . . . . . . . 7

2.2.2 Observer based on solid velocities measurements . . . . . . . . . . . 10

2.2.3 Observer based on solid displacement measurements . . . . . . . . . . 11

2.3 First numerical experiments . . . . . . . . . . . . . . . . . . . . . 13

2.3.1 Example 1 - Stabilization at rest configuration . . . . . . . . . . . 13

2.3.2 Example 2 - Tube with pulsatile flow . . . . . . . . . . . . 15

3 Analysis of the estimators $\quad 18$

3.1 Pure elastodynamics . . . . . . . . . . . . . . . . . . . . 19

3.1.1 Case of solid velocity measurements . . . . . . . . . . . . . . . 20

3.1.2 Case of solid displacement measurements . . . . . . . . . . . . . . 22

3.1.3 Spectral numerical experiments . . . . . . . . . . . . . . . 23

3.2 Added mass effect for elastodynamic coupled with potential flow . . . . . . . . . 23

3.2.1 The added mass effect on stabilization efficiency . . . . . . . . . . . . 24

3.2 .2 Spectral numerical experiments . . . . . . . . . . . . . . . 25

3.2.3 The DVFam filter improvement for solid velocity measurements . . . . . . 26

3.3 Elastodynamics-pressure coupling with lumped-parameter model . . . . . . . . . 27

3.4 Elastodynamics-Stokes coupling . . . . . . . . . . . . . . . . . 32

4 Discussion $\quad 35$

4.1 Choice of the feedback gain in FSI problems . . . . . . . . . . . . . . . . . . 35

4.2 Some inefficient alternative approaches . . . . . . . . . . . . . . . . 35

5 Conclusions and perspectives $\quad 39$

A Properties of the discretization of the harmonic lifitng $\quad 39$

B Spectral sensitivity analysis of the elastodynamics-Windkessel coupling model 40 


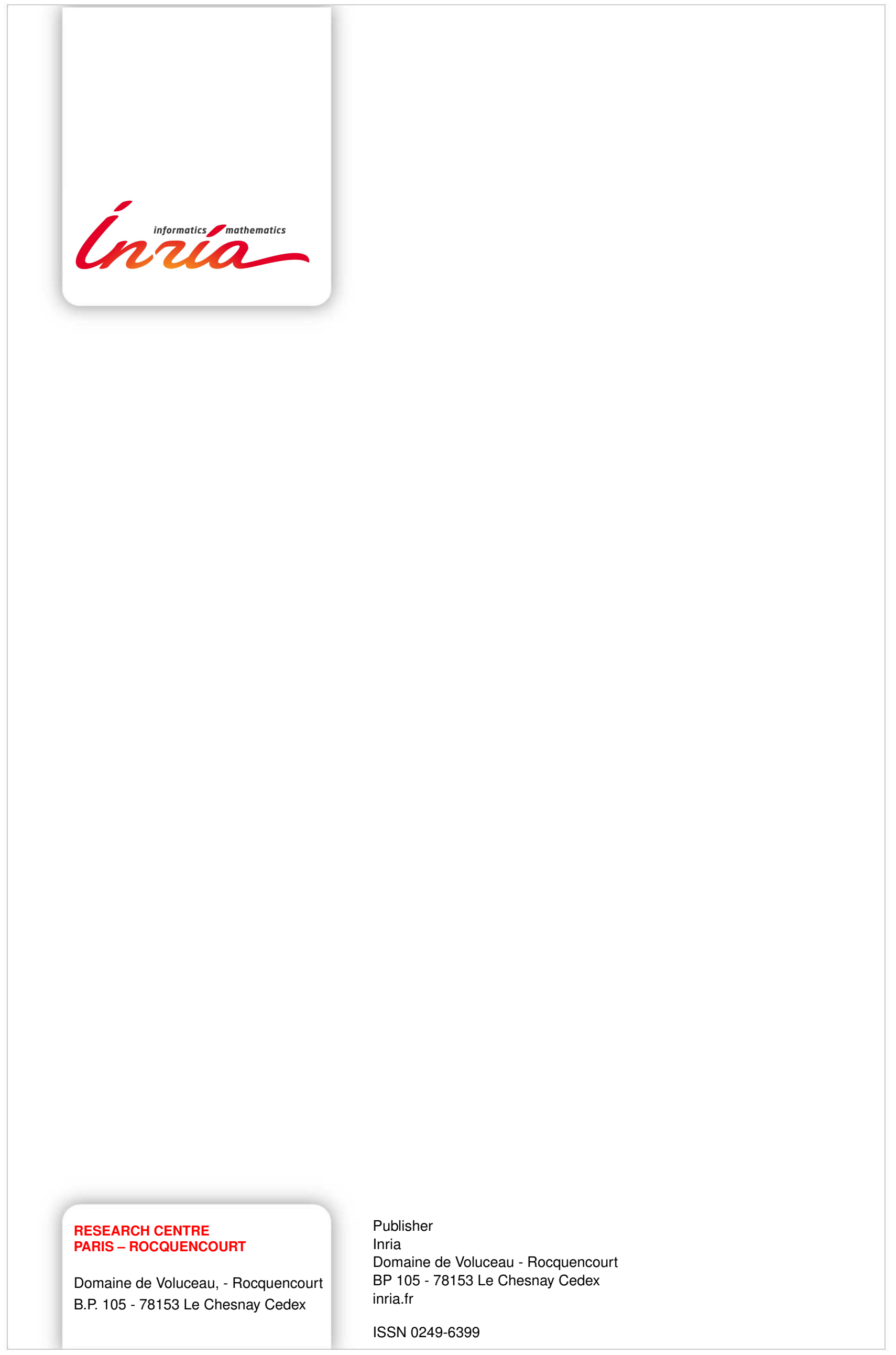

